\title{
ABHANDLUNGEN・ARTICLES・ARTICLES
}

\section{Reformen bei der Beilegung internationaler Investitionsstreitigkeiten - afrikanische Sonderwege?}

\author{
Ludwig Gramlich*
}

\begin{abstract}
Institutions and mechanisms for settlement of international investment disputes are manifold. For sure, rules, principles and core standards for decisions at international level laid down in various documents of public international law are widely agreed upon in general but are rather vague in determining applicable law in specific cases. So, complementing the discussion of the general framework for international investment law in the first part of this study (RiA 2020,3), the focus will now change to the "architecture" of settlement because effective functioning of judicial, arbitral and (other non-judicial) alternative dispute resolution procedures is most important for an attractive climate for sustainable investment. Although there are many similarities between both concepts, it is necessary to separate mechanisms for private (commercial) arbitration from intergovernmental institutions which deal with ISDS issues. Therefore, structures and procedures, forms of settlement and enforcement of awards as well as other final results of dispute resolution concerning foreign investors and investment abroad will be described and assessed more detailedly by focussing in particular upon African examples. Another core problem is balancing the relationship between intergovernmental and non-governmental dispute settlement and national (including supranational) judiciary in order to get proper efficiency. At last, "Africanization" trends as well as other ways for better and more acceptable impartial and fair ISDS will be evaluated which may comply with legitimate expectations of all relevant stakeholders, not least the general public.
\end{abstract}

\section{A. Einleitung}

Im ersten Teil dieses Beitrags ${ }^{1}$ lag der Schwerpunkt der Erörterungen auf materiell-rechtlichen Entwicklungen und Themen. Einige herkömmliche (,klassische“) und erst in den letz-

* Univ.-Prof. i.R. (Technische Universität Chemnitz, Deutschland, Professur für Öffentliches Recht und Öffentliches Wirtschaftsrecht); E-Mail: 1.gramlich@wirtschaft.tu-chemnitz.de.

1 Anmerkung der Redaktion: Der Beitrag von Ludwig Gramlich, Zukunftsfähiger internationaler Rechtsrahmen für Investitionen in Afrika, Recht in Afrika 23 (2020), 3-40, stellt den ersten Teil seiner umfassenden Abhandlung zum Investitionsrecht in Afrika dar. Der vorliegende Artikel mit dem zweiten Teil schließt diese Untersuchung ab. Aufgrund ihres den üblichen Rahmen von Beiträgen in 
ten Jahren verstärkt in den Blick geratene Aspekte sollen im Folgenden noch einmal aufgegriffen und vertieft werden (B.), um die Brücke zu den nunmehr im Mittelpunkt stehenden Organisations- und Verfahrensfragen zu schlagen. Dabei liegt das Hauptinteresse wiederum auf den vielfältigen Schnittmengen, Verknüpfungen und dem personellen, sachlichen, räumlichen und zeitlichen Ineinandergreifen von Institutionen und Mechanismen auf intereinschließlich supranationaler und nationaler Regelungsebene. Die wichtigsten Bausteine und die sie verbindende internationale „Architektur“ werden erneut mit Fokus auf Afrika genauer beschrieben, dabei auch andere außergerichtliche Streitbeilegungs- als Schiedsverfahren behandelt (C., D.) und wird schließlich auch die Phase des „enforcement" näher analysiert (E.). Am Ende wird in einem Fazit diskutiert, ob und wie weit Tendenzen und Schritte hin zu einer "Africanization“ von ,investment settlement disputes" erkennbar sind und ob nicht (auch) andere Reformen geboten oder gar dringlicher erscheinen (G.).Seit Frühjahr 2020 sind insoweit zwar kaum größere rechtliche Änderungen zu verzeichnen, werden jedoch laufende Reformprozesse auch unter Corona-Rahmenbedingungen weiter betrieben. ${ }^{2}$ Die globale COVID 19-Pandemie hat freilich rasch eine Verringerung von internationalen Investitionsströmen ausgelöst, ${ }^{3}$ und es ist zudem absehbar, dass gesundheitspolitisch motivierte einzelstaatliche Maßnahmen zur Eindämmung von Infektionen wegen ihrer enteignungsgleichen Wirkungen nicht nur national verfassungsrechtlich überprüft werden, ${ }^{4}$ sondern auch international auf die Einhaltung der gegenüber ausländischen Investoren und Investitionen eingegangenen Standards (unten, B.) hin. ${ }^{5}$ Diese veränderte Situation betrifft auch und nicht zuletzt Afrika: Von einigen Ausnahmen abgesehen, sind dort vielerorts bewaffnete Konflikte und (andere) politische Unruhen im Gange, formal durchweg demokratische Regierungsformen drohen in etlichen Ländern durch allenfalls halbwegs legale Langzeit-Präsidenten ausgehöhlt zu werden, insbesondere wenn die Regimestruktur an das französische Modell angelehnt ist. Dies kulminiert in zu großen Teilen im Hinblick auf Staatsapparat und damit Durchsetzung ordentlicher Gesetzgebung großflächig kaum existenten ,failed States“, nicht nur am Horn von Afrika, sondern auch in Bereichen der Sahara und

dieser Zeitschrift ausnahmsweise deutlich übersteigenden Umfangs erschien es der Redaktion ratsam, das vom Verfasser behandelte Thema in zwei aufeinander folgenden Heften eines Jahrgangs von Recht in Afrika zu veröffentlichen.

2 Vgl. etwa UNCTAD, International Investment Agreements. Reform Accelerator, 2020, 9 ff.

3 Hierzu UNCTAD, Investment Trends Monitor, Special Issue, März 2020 (Impact of the coronavirus outbreak on global FDI); UNCTAD, Investment Policy Monitor, Special Issue no. 4, Mai 2020 (Investment policy responses to the COVID-19 pandemic); zur Abnahme auch schon zuvor UNCTAD, Investment Trends Monitor no. 31, Jan. 2019 (Global FDI flows continue their slide in 2018); allgemein UNCTAD, World Investment Report 2019, 34 ff.

4 Dazu BVerfG, 11.11.2020, 1 BvR 2530/20; Kersten/Rixen, Der Verfassungsstaat in der Coronakrise, 2020; Frenz, COVuR (COVID-19 und Recht) 2020, 794 ff.; zu ICSID-Verfahren Sanderson, 15.4.2020, https://latinlawyer.com/article/1225491/peru-warned-of-potential-icsid-claims-over-covi d-19-measures.

5 Vgl. den Aufruf unter: http://s2bnetwork.org/offener-brief-zu-isds-und-covid-19/; dazu Gebauer, Frankfurter Rundschau v. 6.11.2020, 16. 
des Sahel. ${ }^{6}$ Die Folgen (volkswirtschaftliche Krisen, Hunger bzw. gravierende Probleme bei der Versorgung mit existenznotwendigen Gütern und Leistungen, Binnenwanderungen auch veranlasst durch ,desertification“ und andere Auswirkungen des Klimawandels ${ }^{7}$ ) zeigen sich seit einiger Zeit auch in Europa, nicht nur wegen der Nachbarschaft am Mittelmeer. Jedenfalls konzeptuell würde das Gros von Wirtschaftsmigranten, aber auch eine Vielzahl von politisches Asyl nachsuchenden Menschen viel lieber in ihrer Heimat bleiben, wenn diese unter ökonomischen, politischen, sozialen, gesellschaftlich-kulturellen Aspekten noch oder wieder lebenswert wäre. Weil freilich „,development assistance“ bzw. „Entwicklungszusammenarbeit" nicht nur im Verdacht stehen, nationale oder ethnische Kulturen nicht angemessen zu respektieren, sondern zudem vielfach noch als bloßes Anhängsel von Außenwirtschafts- bzw. Außenpolitik erachtet werden ${ }^{8}$ und schließlich sowohl auf staatlicher Ebene (als relativ kleiner Teil des jeweiligen Etats ${ }^{9}$ ) als auch auf internationalem Niveau (wegen der bis heute bestehenden Stimmrechtsmacht westlich-atlantischer Staaten, allen voran den USA, und daraus resultierender wirtschaftlich-politischer „Einmischung “10) bei der Verbesserung von Lebensverhältnissen, Wirtschaftsentwicklung und ,governance“ nur eine allgemeine oder ergänzende Rolle spielen können (und auch sollten), ist weltweit nachhaltiges Zusammenleben auf grenzüberschreitende Investitionen insbesondere des privaten Sektors angewiesen, die freilich ohne hierfür günstige Rahmenbedingungen nicht stattfinden oder in lukrativere Räume/Sektoren umgelenkt werden. Da menschliche wirtschaftlichen Aktivitäten bis auf weiteres allein im Bereich des Planeten Erde und dort nur sowohl innerhalb von Territorien in staatlicher Hoheit als auch in internationalisierten Räumen wie etwa Antarktis ${ }^{11}$ und Hoher See ${ }^{12}$ sowie mit dort verfügbaren Ressourcen vor sich gehen können, sind andererseits (restriktive und andere) Verhalten steuernde Maßnahmen unverzichtbar, welche schon heute die Belange auch künftiger Generationen an knappen

6 Vgl. Antrag BÜNDNIS 90/DIE GRÜNEN, Neuausrichtung der europäischen und deutschen Sahelpolitik, BT-Drs. 19/23986 v. 4.11.2020, 1 ff.; weithin positiv hingegen Bundesregierung, Reformpartnerschaft mit Cote d'Ivoire, BT-Drs. 19/23140 v. 6.10.2020, $2 \mathrm{ff}$.

7 Vgl. IPCC, Special report, Climate change and land, 2019, https://www.ipcc.ch/srccl/chapter/chapt er-3/.

8 Vgl. Bundesregierung, Erfolge der deutschen Entwicklungszusammenarbeit in der 19. Legislaturperiode, BT-Drs. 19/23440 v. 15.10.2020, 5 f.; Bundesregierung, IHK-Netzwerk Afrika, BT-Drs. 19/23922 v. 3.11.2020.

9 Vgl. zu aktuellen Daten https://www.oecd.org/dac/financing-sustainable-development/developmen t-finance-standards/official-development-assistance.htm.

10 Lange maßgeblich der von Williamson so bezeichnete Washington Consensus, vgl. https://www.int elligenteconomist.com/washington-consensus/; ferner etwa Bundesregierung, Schuldenerlasse für Entwicklungsländer, BT-Drs. 19/23817 v. 29.10.2020, 2 ff.

11 Vgl. Antarktis-Vertrag v. 1.12.1959, https://www.umweltbundesamt.de/sites/default/files/medien/4 80/dokumente/antarktisvertrag.pdf, Art. I, II, X.

12 Vgl. das Dritte UN-Seerechtsübereinkommen (United Nations Convention on the Law of the Sea, UNCLOS III) v. 10.12.1983, https://www.un.org/Depts/los/convention_agreements/texts/unclos/u nclos_e.pdf, Art. $86 \mathrm{ff}$. 
Ressourcen wahren und dafür sorgen (müssen), dass die mit jeder nicht nur unerheblichen Tätigkeit einhergehenden negativen externen Effekte erkannt und so weit wie möglich vermieden oder doch reduziert werden, wenn und soweit nicht mittels global ansetzender völkerrechtlicher Vorkehrungen, ${ }^{13}$ dann eben regional-kontinental, subregional, bilateral bis herunter zu innerhalb eines Staatswesens eingesetzten Instrumenten und Verfahren auf lokaler Ebene (,glocal“14). Als Beispiel seien nur die Exploration und Abbau von Rohstoffen genannt, bei denen bereits die Gewinnung und Veredelung, aber sodann auch die eigentliche Nutzung (einschließlich etwaiger Wiederverwertung) und schließlich eine ,verträgliche“ Entsorgung mit vielzähligen und vielfältigen internationalen Transaktionen und Investitionen verbunden sind, so dass diese nicht zufällig im Fokus des Energy Charter Treaty ${ }^{15}$ stehen, aber auch (und noch früher) in UNCLOS III einbezogen worden sind (Art. 136 ff., $\left.150 \mathrm{ff}^{16}\right)$.

\section{B. „Klassische“ und neue Regelungen für Investitionen aus dem Ausland und fremde Investoren}

\section{Wichtige Entwicklungsetappen bis heute}

\section{1. „Nördliche“ Investitionen im „Süden“ als Ausgangspunkt}

Bereits die schiere Zahl von über 3.000 bis heute vereinbarten einschlägigen völkerrechtlichen Verträgen ${ }^{17}$ zeigt, dass seit Abschluss und Inkrafttreten der ersten ,bilateral investment treaties" (BITs) Anfang der 60er Jahre des 20. Jahrhunderts ${ }^{18}$ nicht nur deren Bestand rapide gewachsen ist, sondern sich auch wesentliche Parameter geändert haben: Staatliche Parteien sind aktuell nicht mehr fast nur (westliche) Industriestaaten aus dem Mitgliederkreis der $\mathrm{OECD}^{19}$ auf der einen Seite und auf der anderen Entwicklungsländer der südlichen Hemisphäre, vor allem aus Afrika, aber auch aus dem nahöstlichen und weiteren asia-

13 Die 17 Sustainable Development Goals (SDGs), als Teil der 2030 Agenda for Sustainable Development, sind Teil der nicht rechtsverbindlichen Resolution A/RES/70/ der U.N.-Generalversammung v. 25.9.2015, https:/www.un.org/ga/search/view_doc.asp?symbol=A/RES/70/1\&Lang=E; vgl. Huck/Kurkin, ZaöRV 78 (2018), 375 ff.

14 https://en.wikipedia.org/wiki/Glocalization.

15 V. 17.12.1994, https://www.energycharter.org/fileadmin/DocumentsMedia/Legal/1994_ECT.pdf; zum allgemeinen Zweck Art. 2, zu ,investment promotion and protection“ Teil III, Art. $10 \mathrm{ff}$.; dazu Coop, ICSID Review - Foreign Investment Law Journal 29 (2014), 515 ff.; ferner unten, C.III.3.

16 Dazu generell Lehnen u.a., https://mining-report.de/tiefseebergbau-eine-wirtschaftliche-evaluierun $\mathrm{g} /$.

17 Details im Investment Policy Hub von UNCTAD unter https://investmentpolicy.unctad.org/interna tional-investment-agreements.

18 Beginnend mit dem Vertrag zwischen Deutschland und Pakistan v. 25.11.1959 (BGBl. 1961 II, 793), in Kraft seit 28.4.1962.

19 https://www.oecd.org/about/members-and-partners/. 
tischen Raum (allerdings weithin ohne Ozeanien) ${ }^{20}$ und mit einiger Verspätung ${ }^{21}$ aus Latein-, insbesondere Südamerika. Rein verbal waren freilich schon die frühen Investitionsförderungs- und -schutzabkommen auf Gegenseitigkeit angelegt ${ }^{22}$, der Sache nach stammten die ,investments“ (und die betreffenden Investoren) jedoch in der Regel aus ,nördlichen" bzw. „westlichen“ Heimatstaaten, und die Anlagen erfolgten in außereuropäischen Gastländern der „Dritten Welt“ bzw. G 77-Staaten (oder sollten dies jedenfalls ${ }^{23}$ ). Nachdem diese Länder jedoch überdies zahlreich das 1965 geschlossene ICSID-Übereinkommen ${ }^{24}$ ratifizierten - auch hier waren afrikanische Staaten frühzeitig und in großer Anzahl als Vertragsparteien beteiligt ${ }^{25}$-, kam es ab den 70er Jahren zudem zu einer Ergänzung der ursprünglich nur auf ein zwischenstaatliches (schiedsgerichtliches) Streitbeilegungsverfahren $^{26}$ abstellenden prozeduralen Regeln durch zusätzliche Schaffung eines ,investor-State dispute settlement" (ISDS)-Mechanismus. ${ }^{27}$

2. Fester und wachsender Kern von Regelungsstandards - auch zur Förderung von privaten Investitionen in Entwicklungsländern

Zunächst kaum verändert wurde hingegen der inhaltliche Rechtsrahmen des Schutzes grenzüberschreitender Investitionen; insofern gleichen sich bis auf teils abweichenden Aufbau die einschlägigen BITs der EU-Mitgliedstaaten, speziell auch diejenigen mit (west)afri-

$20 \mathrm{Zu}$ den wenigen Ausnahmen zählen die Verträge zwischen Großbritannien und Tonga 1997 oder zwischen Deutschland und Papua-Neuguinea 1980 (BGBl. 1982 II, 389), vgl. https://investmentpol icy.unctad.org/international-investment-agreements; für die Region abgeschlossen, aber noch nicht in Kraft Pacific Agreement on Closer Economic Relations (PACER) Plus 2017, https://investment policy.unctad.org/international-investment-agreements/treaty-files/5586/download.

21 Beispiele sind Verträge Deutschlands mit Panama 1983 (BGBl. 1987 II, 2), Uruguay 1987 (BGBl. 1988 II, 272), Guyana 1989 (BGBl. 1993 II, 938); zwischen Frankreich und Panama 1982, Argentinien 1991 oder Uruguay 1993; von Portugal mit Venezuela und Peru 1994; zwischen Spanien und Chile und Argentinien 1991; https://investmentpolicy.unctad.org/international-investment -agreements.

22 Vgl. nur die Präambel des deutsch-pakistanischen Vertrags 1959 (Fn. 18), die von Schaffung „günstiger Bedingungen für Kapitalanlagen von Staatsangehörigen und Gesellschaften des einen Staates im Hoheitsgebiet des anderen Staates" spricht und dieses Konzept dann in Art. 1 Abs. 1 bekräftigt; ferner unten, bei Fn. 30 .

23 „Der Handelsaustausch hat ein relativ geringes Volumen und trotz des bilateralen Investitionsschutzabkommens von 1977 gibt es keine wesentlichen deutschen Investitionen in Mali. Mali ist Unterzeichner des Cotonou-Abkommens mit der Europäischen Union“; https://www.auswaertigesamt.de/de/aussenpolitik/laender/mali-node/bilateral/208244.

24 V. 18.3.1965 (zur Beilegung von Investitionsstreitigkeiten zwischen Staaten und Angehörigen anderer Staaten, BGBl. 1969 II, 369), https://investmentpolicy.unctad.org/international-investment-a greements/treaty-files/2766/download.

25 https://icsid.worldbank.org/about/member-states; speziell zu Afrika unten, C.II.1.b).

26 Vgl. etwa Art. 11 des Vertrags zwischen Deutschland und Togo v. 16.5.1961 (BGBl. 1964 II, 154).

27 Deutschland erstmals in Art. 10 Abs. 6 des BIT mit Somalia v. 27.11.1981 (BGBl. 1984 II, 778); als eigener Art. 11 im BIT mit Kap Verde v. 18.1.1990 (BGB1. 1993 II, 947). 
kanischen Ländern. So hat etwa Deutschland mit 14 der 17 OHADA-Mitglieder ${ }^{28}$ Investitionsschutzverträge abgeschlossen, die meisten bereits in den 60er Jahren ${ }^{29}$ - durchweg ,in dem Wunsch, die wirtschaftliche Zusammenarbeit zwischen beiden Staaten zu vertiefen, in dem Bestreben, günstige Bedingungen für die Anlage von Kapital durch Staatsangehörige und Gesellschaften des einen Staats im Hoheitsgebiet des anderen Staates zu schaffen, und in der Erkenntnis, dass eine Förderung und ein vertraglicher Schutz dieser Kapitalanlagen geeignet ist, die private wirtschaftliche Initiative zu beleben und den Wohlstand beider Völker zu mehren“. ${ }^{30}$ Dieses in der Abkommens-Präambel formulierte Programm wurde für (damals noch nicht im [Eingangs-]Art. 1, ${ }^{31}$ sondern erst nach den einzelnen Standards definierte) „Kapitalanlagen“ und „Erträgnisse“ im Hinblick auf jeweilige „Staatsangehörige“ und „Gesellschaften“ präzisiert (Art. 8), ${ }^{32}$ bezogen auf die generelle „Förderung“ und wohlwollende Handhabung der "Zulassung“ (nach Maßgabe gaststaatlichen Rechts), Art.2 Abs. 1. Den Kern eines Vertrags bilde(te)n die Pflicht zu Inländer(gleich)- (national treatment, NT) und Meistbegünstigungsbehandlung (most favoured nation treatment, MFN) von Investitionen und Investoren (auch bei Verwaltung, Gebrauch und Nutzung der Kapitalanlagen, Art. 2 Abs. 2, sowie zudem bei Entschädigungen für Enteignungen - bzw. Verstaatlichungen oder beiden in ihrer Auswirkung gleichkommenden Maßnahmen - und für Verluste durch bewaffnete Konflikte, je einschließlich zugehöriger Erträge, Art. 3 Abs. 2 - 5), die Gewährleistung vollen Schutzes und voller Sicherheit (full protection and security, FPS; Art. 3 Abs. 1) sowie die Freiheit des (Rück-)Transfers von Kapital (mitsamt der Erträgnisse und auch in Bezug auf einen Liquidationserlös, Art. 4, 6). Häufig war Vertragsbestandteil auch ein Protokoll zur Auslegung einzelner Vorschriften. ${ }^{33}$ Zur generellen Begründung verlautete dabei, Deutschland sei bestrebt, „durch günstige Bedingungen die private wirtschaftliche Initiative zu Kapitalanlagen in Entwicklungsländern zu beleben. Die Förderung privater Auslandsinvestitionen erscheint als geeignetes Mittel, den wirtschaftlichen Aufbau in den Entwicklungsländern durch die Zuführung von Geld und Sachkapital in Verbindung mit wissenschaftlichen Erkenntnissen, technischen Erfindungen und Verfahren über die wirksamste Anlage des Kapitals zu begünstigen“. Mit diesem Zweck sei ein bilaterales Abkommen zudem ,geeignet“, die zwischen den Parteien „bestehenden vertrauensvollen wirt-

28 Außer Äquatorial-Guinea, Guinea-Bissau, Komoren.

29 Togo 1961 (BGB1. 1964 II, 154), Guinea 1962 (BGB1. 1964 II, 145), Kamerun 1962 (BGB1. 1963 II, 991), Senegal 1964 (BGBl. 1965 II, 1391), Niger 1964 (BGB1. 1965 II, 1402), Republik Kongo 1965 (BGB1. 1966 II, 841), Zentralafrikanische Republik 1965 (BGBl. 1967 II, 1657), https://inves tmentpolicy.unctad.org/international-investment-agreements/.

30 So etwa in der Präambel des Vertrags zwischen Deutschland und der Elfenbeinküste v. 27.6.1966 (BGB1. 1968 II, 61).

31 Wie etwa im Vertrag mit Mali v. 28.6.1977 (BGB1. 1979 II, 77).

32 Etwa in den BITs mit Marokko v. 31.8.1961 (BGB1. 1967 II, 1641); mit Liberia v. 12.12.1961 (BGB1. 1967 II, 1537); mit Madagaskar (alt) v. 21.9.1962 (BGB1. 1965 II, 370).

33 Etwa im Fall von Mali, dazu BT-Drs. 8/1743 v. 2.10.1978, 15. 
schaftlichen Beziehungen weiter zu vertiefen“. ${ }^{34}$ Erweiternd und vertiefend hieß es in den 1970er Jahren etwa zum Vertrag mit Mali, die Bundesrepublik Deutschland unterstütze den wirtschaftlichen Aufbau der Entwicklungsländer einerseits „durch verschiedene Maßnahmen der öffentlichen Hand“. Jedoch sei die „Bereitstellung öffentlicher Mittel für diesen Zweck aus dem Bundeshaushalt naturgemäß begrenzt. Deshalb ist die Bundesregierung bestrebt, private Kapitalanlagen in Entwicklungsländern zu fördern“. Zu diesem Zweck eingesetzte „Garantien, Steuervergünstigungen und Kredite“ dienten „gleichzeitig der Anregung des deutschen Kapitalexports und der Intensivierung der außen-wirtschaftlichen Beziehungen zwischen der Bundesrepublik Deutschland und den jeweiligen Partnerländern“. Denn „Investitionen der privaten Wirtschaft führen in der Regel neben dem Zufluss von Geld oder Sachwerten auch gleichzeitig zur Vermittlung technischen Wissens und technischer Erfahrung durch geeignete Fachkräfte, die in den Entwicklungsländern besonders wertvolle Arbeit leisten. Private Kapitalanlagen haben den Vorzug, daß mit dem Kapital auch die unternehmerische Erfahrung investiert wird, und daß das unternehmerische Risiko der Kapitalanlagen in vollem Umfang vom Investor getragen wird“. Als „Ziel“ des Abkommens wurde hervorgehoben, dass die Anlage privaten deutschen Kapitals „durch die Sicherung eines ausreichenden Rechtsschutzes auf der Grundlage völkerrechtlicher Verträge besonders gefördert werden" solle. Betont wurde zudem, ebenso wie die mit anderen Staaten getroffenen Vereinbarungen gleicher $\mathrm{Art}^{35}$ lehne sich der Vertrag ,auf der Grundlage der Gegenseitigkeit inhaltlich an die von der Bundesregierung abgeschlossenen Freundschaft-, Handels- und Schiffahrtsverträge ${ }^{36}$ an, soweit sie sich mit der Frage der Nichtdiskriminierung auf wirtschaftlichem Gebiet und mit dem Vermögensschutz im Falle einer Enteignung befassen“. ${ }^{37}$ Zur ebenfalls regelmäßig vorhandenen „Subrogations“"-Klausel ${ }^{38}$ wurde erläutert, Kapitalschutzabkommen trügen zugleich den Bestimmungen des (Bundes-)Haushaltsgesetzes $^{39}$ Rechnung, nach denen „der Bund zur Absicherung des politischen Risikos bei förderungswürdigen Kapitalanlagen im Ausland Bürgschaften, Garantien und sonstige Gewährleistungen in der Regel dann übernehmen (darf), wenn zwischen der Bundesrepublik Deutschland und dem Land, in dem die Kapitalanlage vorgenommen wird, eine Vereinbarung über die Behandlung von Kapitalanlagen besteht“. Daher werde „die Bundesregie-

34 So zu Madagaskar, BT-Drs. IV/2636 v. 19.10.1964, 17.

35 Vgl. die Nachweise in den vorhergehenden Fußnoten; ferner etwa BIT mit Benin v. 29.6.1978 (BGBl. 1985 II, 2).

36 Wie zwischen Deutschland und den USA (29.10.1954, BGBl. 1956 II, 487); BGH, 29.1.2003, VIII ZR 155/02, $7 \mathrm{f}$.

37 BT-Drs. 8/1743, 14.

38 Beziehungsweise „Gläubigerwechsel“; dazu noch unten, Fn. 53, 228.

39 Bis heute, jüngst $\S 3$ des Haushaltsgesetzes 2021; dazu BT-Drs. 19/22600 v. 25.9.2020, 11, 17; s. bereits Begründung zu $\S 17$ Entwurf des Haushaltsgesetzes 1959, BT-Drs. III/650 v. 1.12.1958, 6 f. Dazu Bundesregierung, Bestimmungen zum Investitionsschutz und Investor-Staat-Schiedsverfahren in bilateralen Investitionsschutzabkommen, BT-Drs. 18/7753 v. 2.3.2016, 11; zur Praxis PwC, Investitionsgarantien, Jahresbericht 2019, https://www.investitionsgarantien.de/_Resources/Persist ent/f44997b60183de87186f45daf705837d868a508b/DIA\%20Jahresbericht\%202019.pdf. 
rung, wenn sie auf Grund einer Gewährleistung für eine“ im anderen Vertragsstaat „,vorgenommene Kapitalanlage in Anspruch genommen wird, in die Lage“ versetzt, die auf sie übergegangenen Rechte des Kapitalanlegers" im eigenen Namen geltend zu machen; dabei finden ,auf Zahlungen, die auf Grund übertragener Rechte und Ansprüche zu leisten sind“, die Bestimmungen über den freien Transfer ebenfalls Anwendung. ${ }^{40}$ Weitere Standard-Vorschriften beinhalten eine „Besserstellungsklausel“, ${ }^{11}$ nach welcher ,günstigere Regelungen, die eine Vertragspartei in Bezug auf eine den Bestimmungen des Vertrages unterliegende Kapitalanlage übernommen hat, z.B. im Wege einer Konzession oder einer privatrechtlichen Vereinbarung, den Bestimmungen des Vertrages“ vorgehen, und „die Verletzung einer solchen Verpflichtung hiernach zugleich eine Verletzung der durch den vorliegenden Vertrag übernommenen völkerrechtlichen Verpflichtung“ darstellt. Im Haupttext ${ }^{42}$ oder einem Protokoll wird (nicht nur bei Binnenstaaten) auch oft eine Verpflichtung der Vertragspartner normiert, alle Maßnahmen zu unterlassen, die geeignet sind, die Freiheit des Investors hinsichtlich der Auswahl der Transportmittel zu beschränken oder zu behindern. Weitere Differenzierungen und Präzisierungen erfolgten dann Ende des 20. Jahrhunderts, teils auch im Rahmen eines neuen, den bisherigen ablösenden BIT. So wird im Abkommen mit Gabun $1998^{43}$ der anderen Seite bzw. deren Investoren in jedem Fall gerechte und billige Behandlung (fair and equal treatment, FET) zugesichert (Art. 2 Abs. 1 S. 2), und im folgenden zweiten Absatz wird statuiert, die eine Vertragspartei werde ,die Verwaltung, die Verwendung, den Gebrauch oder die Nutzung der Kapitalanlagen von Staatsangehörigen oder Gesellschaften der anderen Vertragspartei in ihrem Hoheitsgebiet in keiner Weise durch willkürliche oder diskriminierende Maßnahmen beeinträchtigen“ (ebenso Art. 2 Abs. 3 des BIT mit Guinea ${ }^{44}$ ). Ferner wird die Anwendung der MFN-Klausel begrenzt; sie beziehe sich „nicht auf Vorrechte, die eine Vertragspartei den Staatsangehörigen oder Gesellschaften dritter Staaten wegen ihrer Mitgliedschaft in einer Zoll- oder Wirtschaftsunion, einem gemeinsamen Markt oder einer Freihandelszone oder wegen ihrer Assoziierung damit einräumt“ (Art. 3 Abs. 3). Für „Vergünstigungen, die eine Vertragspartei den Staatsangehörigen oder Gesellschaften dritter Staaten aufgrund eines Doppelbesteuerungsabkommens oder sonstiger Vereinbarungen über Steuerfragen gewährt“, finde auch Inländerbehandlung keine Anwendung (Art. 3 Abs. 4). Die Besteuerungs-Ausnahme wurde in anderen Verträgen noch breiter gefasst (etwa mit Libyen, Art. 3 Abs. 5, 6). Explizite Verdeutlichungen betreffen vor allem die Formen von Investor-"Betätigungen" sowie exemplarische Regelungen über ,weniger günstige“ Behandlungen (in Bezug auf NT- oder MFN-Vorschriften) und davon nicht erfasstes Verhalten, wie ,aus Gründen der öffentlichen Sicherheit und Ordnung,

40 BT-Drs. 8/1743, 14, 15.

41 So BR-Drs. 832/08 v. 7.11.2008, 13, zum BIT mit Libyen v. 15.10.2004 (BGB1. 2009 II, 462).

42 So Art. 3 Abs. 7 BIT mit Guinea (neu) v. 8.11.2006 (BGB1. 2008 II, 487), welcher den Vertrag v. 19.4.1962 ersetzte (Art. 13 Abs. 3).

43 V. 15.9.1998 (BGBl. 2001 II, 478), zugleich den BIT v. 16.5.1969 (BGB1. 1970 II, 657) ablösend (Art. 12 Abs. 4).

44 Fn. 42. 
der Volksgesundheit oder Sittlichkeit" zu treffende Maßnahmen. Schließlich werden teils Wohlwollensregeln im Hinblick auf Einreise, Aufenthalt und Arbeitserlaubnis sowohl für (selbstständige) Personen als auch speziell für Arbeitnehmer des „Heimatstaats“ im Zusammenhang mit der Investition im Gastland getroffen, freilich allein im Rahmen des dort jeweils geltenden innerstaatlichen Rechts. ${ }^{45}$ Letztlich nur klarstellend ist auch die Bestimmung, dass „Einkünfte aus der Kapitalanlage und im Fall ihrer Wiederanlage auch deren Einkünfte“ den gleichen Schutz wie die Investition selbst genießen. ${ }^{46}$ Auch wird zuweilen angeführt, falls erforderlich, würden ,Repräsentanten der Vertragsstaaten zu Fragen der Anwendung des Abkommens Konsultationen führen“, 47 während die noch im (letzten) deutschen Vertragsmuster $2009^{48}$ (in Art. 12) enthaltene Registrierungsklausel (betr. Art. 102 $\mathrm{UN}-\mathrm{Charta}^{49}$ ) anscheinend nirgends erwähnt wird.

\section{Einbeziehung von ISDS-Klauseln}

In den 1990er Jahren wurden in neue oder erneuerte BITs Deutschlands mit OHADA-Staaten auch Vorschriften zu einem Investor-Staat-Streitbeilegungsverfahren aufgenommen, insoweit erstmals im Abkommen mit Burkina Faso $1996^{50}$ in Art. 11, ohne dass diesem Umstand bei der Behandlung des Vertragsgesetzes im Bundestag größere Beachtung geschenkt wurde und die Entwurfsbegründung lapidar vermerkte, dieser Artikel sehe ,eine internationale Schiedsgerichtsbarkeit bei Meinungsverschiedenheiten zwischen einem Investor und dem jeweiligen Gaststaat vor“ ${ }^{51}$ Die Neuerung gab auf, zunächst soweit möglich eine ,gütliche“ Beilegung zwischen den Streitparteien anzustreben, nach Verstreichen einer Frist von sechs Monaten aber dem Investor ein Recht auf Einleitung eines Schiedsverfahrens zu geben, für welches die Vorschriften des ICSID-Übereinkommens gelten sollten, soweit Investor und Gaststaat keine abweichende Vereinbarung treffen. Ausdrücklich normiert wurde zudem, der Schiedsspruch sei „,bindend“, unterliege keinen anderen als den im ICSID-Abkommen vorgesehenen „Rechtsmitteln oder sonstigen Rechtsbehelfen“ und werde „,nach innerstaatlichem Recht vollstreckt". ${ }^{52}$ Schließlich wurde als Abrundung der Subrogation ${ }^{53}$ festgestellt, die ,an der Streitigkeit beteiligte Vertragspartei“ werde „während eines

45 Art. 3 Abs. 3 und 7 BIT mit Libyen 2004; Art. 3 Abs. 2 und 6 BIT mit Guinea 2006.

46 Art. 2 Abs. 4 BIT mit Guinea 2006.

47 Art. 12 BIT mit Guinea 2006.

48 https://investmentpolicy.unctad.org/international-investment-agreements/treaty-files/2865/downlo ad; Muster 2005 bei: Ceyssens/Sekler, Bilaterale Investitionsabkommen (BITs) der Bundesrepublik Deutschland, 2005, $132 \mathrm{ff}$.

$49 \mathrm{Zu}$ Durchführungsregeln der U.N.-Generalversammlung: https://treaties.un.org/xml/db/MSDB/pag eRegulation_en.

50 V. 22.10.1996 (BGB1. 1998 II, 1458); s. bereits oben, Fn. 28.

51 BT-Drs. 13/9959 v. 17.2.1998, 16.

52 Zur Verbindung zur Vollstreckung näher unten, E.

53 S. schon oben, bei Fn. $38 \mathrm{ff}$. 
Schiedsverfahrens oder der Vollstreckung eines Schiedsspruchs nicht als Einwand geltend machen, dass der Staatsangehörige oder die Gesellschaft der anderen Vertragspartei eine Entschädigung für einen Teil des Schadens oder den Gesamtschaden aus einer Versicherung erhalten hat".

\section{Zwischen Bewährung und Reform}

Keine BITs bestehen zwischen Deutschland und drei OHADA-Ländern ${ }^{54}$ sowie mit Dschibuti, Eritrea, Gambia, Malawi, São Tomé und Príncipe und den Seychellen. ${ }^{55}$ Noch 2014 verlautbarte die Bundesregierung, die Regelungen für Investor-Staat-Schiedsverfahren in deutschen bilateralen Investitionsförder- und -schutzverträgen mit Entwicklungs- und Schwellenländern hätten sich bewährt: „Sie haben Investoren in Ländern mit rechtsstaatlichen Defiziten in ihrer Rechtsordnung oder ihrem Rechtsschutz die Möglichkeit gegeben, diskriminierende oder willkürliche staatliche Beeinträchtigungen ihrer Investitionen auf völkerrechtlicher Basis abzuwehren. Vielfach haben Entwicklungs- und Schwellenländer ihrerseits die Bundesrepublik Deutschland um den Abschluss eines Investitionsförder- und -schutzvertrags gebeten, um ihr Land attraktiver für ausländische Investoren zu machen.“ Über die Notwendigkeit und Ausgestaltung der BITs habe die Bundesregierung jeweils im Einzelfall entschieden. ${ }^{56}$ In jenem Jahr hieß es aber auch (im Rahmen der TTIP-Diskussi$\mathrm{on}^{57}$ ), die Regierung sehe „,keine Notwendigkeit für die Einbeziehung von Regelungen zum Investitionsschutz und Investor-Staat-Schiedsverfahren im Abkommen, da EU-Investoren in den USA und US-Investoren in Deutschland hinreichenden Schutz vor nationalen Gerichten haben“. ${ }^{58}$ Generell wurden zu jener Zeit Ansatzpunkte für Verbesserungen des Investor-Staat-Schiedsverfahrens benannt, nämlich „die diversen Schiedsverfahrensordnungen (wie z.B. UNCITRAL ${ }^{59}$ und ICSID Arbitration Rules ${ }^{60}$ ), auf welche die bilateralen In-

54 Oben, Fn. 28.

55 Vgl. https://investmentpolicy.unctad.org/international-investment-agreements/countries/78/german $\mathrm{y}$.

56 Vgl. Bundesregierung, Erfahrungen, Bedeutung und zukünftiger Umgang mit Investor-StaatSchiedsgerichtsverfahren als Teil von bilateralen Freihandelsabkommen, BT-Drs. 18/1120 v. 10.4.2014, 3 .

57 Vgl. schon Bundesregierung, Freihandels- und Investitionsabkommen zwischen der Europäischen Union und den USA, BT-Drs. 17/13070 v. 15.4.2013, 1 ff.; Beck/Ohr, Wirtschaftsdienst 2014, 344 ff.; Treier/Wernicke, EuZW 2015, 334 ff.; Hummer, integration 2015, 3 ff.; Classen, in: Ludwigs/Remien (Hrsg.), Investitionsschutz, Schiedsgerichtsbarkeit und Rechtsstaat in der EU, 2018, 59 ff., aber auch $V o \beta$, Brauchen Investitionen im TTIP Schutz?, 2014, $27 \mathrm{ff}$.

58 Bundesregierung, Soziale, ökologische, ökonomische und politische Effekte des EU-USA-Freihandelsabkommens, BT-Drs. 18/2100 v. 10.7.2014, 3.

59 https:/www.uncitral.org/pdf/english/texts/arbitration/arb-rules-2013/UNCITRAL-Arbitration-Rul es-2013-e.pdf.

60 https://icsid.worldbank.org/resources/rules-and-regulations/convention/arbitration-rules (ICSID/15, April 2006). 
vestitionsförder- und -schutzverträge der Bundesrepublik Deutschland verweisen. So wurden zur Stärkung der Transparenz von Investor-Staat-Schiedsgerichtsverfahren beispielsweise bereits im Jahr 2006 die Arbitration Rules zur ICSID-Konvention um zusätzliche Bestimmungen ${ }^{61}$ ergänzt, und auch im Rahmen der UNCITRAL fänden Beratungen mit gleicher Zielsetzung statt, die teils (2014) bereits abgeschlossen seien. ${ }^{62} \mathrm{Zu}$ Sachthemen verlautet, der Schutz gegen „,indirekte Enteignungen“ in BITs könne „so ausgestaltet werden, dass allgemeine und angemessene Regelungen zum Schutz von Gemeinwohlzielen, die in demokratischen Entscheidungen rechtsstaatlich zustande kommen, nicht ausgehebelt und umgangen werden;“ auch ein bloßes Investitionsschutzkapitel innerhalb eines umfassenderen Abkommens dürfe daher ,nicht so ausgestaltet werden, dass dieser gesetzgeberische Spielraum eingeschränkt wird."63 Wenig später fasste die Bundesregierung zusammen, eine UNCTAD-Auswertung zahlreicher empirischer Studien zum Einfluss von Internationalen Investitionsschutzabkommen auf ausländische Direktinvestitionen für den Zeitraum von 1987 bis $2014^{64}$ zeige, dass Investitionsschutzverträge positive Auswirkungen auf ausländische Direktinvestitionen haben. Darüber hinaus bestehe „Einigkeit, dass weitere Faktoren wie die Höhe des politischen Risikos, die Entwicklung des Finanzsektors, steuerliche Regelungen, Umwelt- und Arbeitsmarktstandards die Investitionsentscheidung beeinflussen.“ Investitionsschutzverträge ,mit Ländern mit Defiziten im Rechtsschutz“ seien daher „,weiterhin ein wichtiger Bestandteil der Außenwirtschaftspolitik“, denn sie „fördern die Vorhersehbarkeit, Stabilität und Transparenz, reduzieren die Risiken für Investoren und tragen zu einem besseren Investitionsklima bei." ${ }^{65}$ Dass je nach Bedeutung der anderen Vertragspartei aber auch Nicht-OECD-Staaten durchaus besondere Konditionen erhalten konnten, zeigt der deutsch-chinesische BIT von 2003, ${ }^{66}$ wo (etwas versteckt im Protokoll) Modifizierungen wesentlicher Standards allein zugunsten einer Seite (der chinesischen) vereinbart wurden.

61 Ursprüngliche Fassung 1968: https://icsid.worldbank.org/sites/default/files/ICSID\%20Regulations \%20and\%20Rules\%201968\%20-\%20ENG.pdf. Zu den Änderungen 2006 vgl. Antonietti, ICSID Review - Foreign Investment Law Journal 21 (2006), 427 ff.

62 BT-Drs. 18/1120, 3.

63 BT-Drs. 18/1120, 5; kritisch BT-Drs. 18/1964 v. 2.7.2014 (Antrag BÜNDNIS 90/DIE GRÜNEN).

64 UNCTAD-Issue Note, The Impact of International Investment Agreements on Foreign Direct Investment: An Overview of Empirical Studies 1998-2014, Sept. 2014.

65 Bundesregierung, Investitionsschutz in bilateralen Handelsverträgen zwischen der Bundesrepublik Deutschland und Entwicklungs- bzw. Schwellenländern, BT-Drs. 18/4523 v. 31.3.2015, 21.

66 1.12.2003 (BGBl. 2005 II, 733); dazu Tillmann/Braun/Schonard, ICSID Review - Foreign Investment Law Journal 22 (2007), 258 ff. 
5. Gleichklang der Regelungen Deutschlands und anderer EU-Staaten

a) Weitreichende Übereinstimmung

Die in von Deutschland abgeschlossenen BITs enthaltenen Vorschriften sind weder insgesamt noch in den wesentlichen Einzelheiten eine germanische Besonderheit - im Gegenteil, sie stellen bis heute den inhaltlichen Kern von BITs und anderen, international investment agreements" (IIAs) dar. Maßgebliche materielle Regelungen (substantive provisions) bezwecken durchweg die Gewährleistung von Standards für die Behandlung von Investoren (FET, MFN, NT, FPS ${ }^{67}$ ) und legen das Augenmerk bei der Einhaltung von Verpflichtungen auf das Verhalten der (gast)staatlichen Seite. Hierfür zentrale Konzepte stammen schon aus dem 19. bzw. der ersten Hälfte des 20. Jahrhunderts und wurden nicht zuletzt im Verhältnis zwischen Nord- und Mittel-/Südamerika als fremdenrechtliche Mindestanforderungen formuliert, ebenso wie die Gegenposition der Calvo-Doktrin, Ausländern sei keine bessere Behandlung geschuldet als den eigenen Staatsangehörigen, weder materiell noch prozedural. ${ }^{68}$

b) Wichtige Details

Andere europäische Staaten haben nach Aufbau und Inhalten sehr ähnliche Verträge mit Ländern des OHADA-Raums wie des übrigen Afrikas abgeschlossen. Dort finden sich bereits in den 1980er Jahren ISDS-Klauseln, die wie in der deutschen Praxis mit der Subrogation (bei Eintritt einer [heimat]staatlichen Investitionsversicherung) verknüpft sind, etwa in Art. 7 - 9 des Abkommens zwischen Frankreich und Äquatorial-Guinea ${ }^{69}$ oder in Art. 8, 10 des Vertrags zwischen Benin und dem Vereinigten Königreich. ${ }^{70}$ Art. 8 Abs. 2 dieses BIT schreibt dabei vor: „Neither Contracting Party shall pursue through the diplomatic channel any dispute referred to the Centre unless:

(a) the Secretary-General of the Centre, or a conciliation commission or an arbitral tribunal constituted by it, decides that the dispute is not within the jurisdiction of the Centre; or

(b) the other Contracting Party should fail to abide by or to comply with any award rendered by an arbitral tribunal."

67 Vgl. oben, 2.; zur ökonomischen Wirkung Berger et al., More Stringent BITs, Less Ambitious Effects on FDI? Not a Bit!, WTO Staff Working Paper ERSD-2010-10. Mai 2010.

68 Vgl. schon Gramlich, RiA 2020, 3 (27 f.); neuerdings etwa Bundesregierung, Bestimmungen zum Investitionsschutz und zum Investor-Staat-Schiedsverfahren in bilateralen Investitionsschutzabkommen: BT-Drs. $18 / 7753$ v. 2.3.2016, 1 ff., aber auch Ceyssens/Sekler (Fn. 48), 22 f.; Alvarez, Alabama Law Review 60 (2009), 943 (959 ff.).

69 V. 3.3.1982; https://investmentpolicy.unctad.org/international-investment-agreements/treaty-files/1 141/download.

70 V. 27.11 .1987 , https://investmentpolicy.unctad.org/international-investment-agreements/treaty-file s/4775/download. 
Häufig, wenn auch im Detail unterschiedlich sind weiterhin Klauseln dazu, welche Regelungen vorrangig anwendbar sind, etwa in Art. 7 des BIT zwischen Benin und der BelgischLuxemburgischen Wirtschaftsunion (BLEU) 2001:71 „Lorsqu'une question relative aux investissements est regie à la fois par le present Accord et par la législation nationale de l'une des Parties contractantes ou par des conventions internationales en vigueur actuellement ou contractées dans l'avenir par les Parties, les investisseurs de l'autre Partie contractante pourront se prévaloir des dispositions qui leur sont les plus favorables." Dieses Abkommen enthält im Anschluss (in Art. 8) auch eine spezielle ,umbrella clause“, ${ }^{72}$ zum einen im Sinne eines Nebeneinander (Abs. 1: „Les investissements ayant fait l'objet d'un accord particulier entre l'une des Parties contractantes et des investisseurs de I'autre Partie seront régis par les dispositions du present Accord et par celles de cet accord particulier"), zum anderen als Schutz vor hoheitlichem Vertragsbruch (Abs. 2: „Chacune des Parties contractantes s'engage à assurer à tout moment le respect des obligations qu'elle aura contractées à l'egard des investisseurs de l'autre Partie contractante"). Im Hinblick auf ISDS-Verfahren werden teils eingehendere Vorgaben getroffen: So ist zwischen Benin und der BLEU vorgesehen, dass die zunächst anzustrebende Verhandlungslösung auch von statten gehen kann „en faisant éventuellement appel à l'avis specialisé d'un tiers, ou par la conciliation entre les Parties contractantes par la voie diplomatique“ (Art. 9 Abs. 1). Für die mangels einer einvernehmlichen Regelung folgende zweite Stufe bestimmt Art. 9 Abs. 2, die Streitigkeit werde nach Wahl des Investors unterbreitet ,soit à la juridiction compétente de l'Etat ou l'investissement a été realisé, soit à l'arbitrage international." Zu diesem Zweck gebe jede Vertragspartei „son consentement anticipé et irrevocable à ce que tout différend soit soumis à cet arbitrage“, was auch den Verzicht auf „l'épuisement des recours administratifs ou judiciaires internes" bedeute. Diverse Optionen für ein internationales Schiedsverfahren eröffnet dem Investor Art. 9 Abs. 3: ein ad hoc-Schiedsgericht, das nach den UNCITRAL-Regeln verfährt, den ICSID-Mechanismus, wenn beide Abkommensparteien Mitglied dieser Einrichtung sind, bzw. bis dahin eine Nutzung der ICSID Additional Facility, ${ }^{73}$ ferner das ICC-Schiedsgericht Paris ${ }^{74}$ und das einschlägige Institut der Stockholmer Handelskammer (SCC). ${ }^{75}$ Schließlich enthält dieser BIT in Art. 9 Abs. 5 noch eine Aussage zum anwendbaren Recht: „Le tribunal arbitral statuera sur la base du droit interne de la Partie contractante partie au différend sur le territoire de laquelle l'investissement a été realisé, y compris les règles relatives aux conflits de lois, ainsi que sur la base des dispositions du présent Accord, des termes de l'accord particulier éventuellement conclu au sujet

71 V. 18.5.2001, https://investmentpolicy.unctad.org/international-investment-agreements/treaty-files/ 327/download.

$72 \mathrm{Zu}$ deutschen BITs s. Übersicht in BT-Drs. 18/7753, 4 ff.; ferner Schill, Enabling private ordering, IILJ Working Paper 2008/9, 26 ff.

73 https://icsid.worldbank.org/resources/rules-and-regulations/additional-facility-rules/overview.

$74 \mathrm{https}$ //iccwbo.org/dispute-resolution-services/icc-international-court-arbitration/.

$75 \mathrm{https}: / / \mathrm{sccinstitute.com} /$. 
de l'investissement et des principes de droit international." Der zwischen Benin und den Niederlanden bestehende Vertrag (ebenfalls von 2001) ${ }^{76}$ verhält sich hingegen zu ISDS überaus wortkarg, sieht allein den Weg zum ICSID-Verfahren vor, wobei allerdings außer „arbitration“ auch Schlichtung (conciliation) aufgeführt und sodann speziell die Konstellation des Art. 25 Abs. 2 lit. b) des ICSID-Abkommens aufgegriffen wird, dass ,une personne morale ressortissante de l'une des Parties Contractantes et que, avant l'apparition du différend, est contrôlée par des ressortissants de l'autre Partie Contractante“, als Gesellschaft dieser letztgenannten Seite zu erachten sei (Art. 9). Kamerun und Italien haben $1997^{77}$ als Alternativen zu Streitbeilegung auf internationaler Ebene auch vorgesehen das Einlegen eines Rechtsbehelfs zu Verwaltungsbehörden oder Gerichten des (Vertrags-)Staates, in welchem die Investition getätigt wurde (Art. 7 Abs. 2 lits. a], b]). Für die Dauer eines Schiedsoder Gerichtsverfahrens verpflichten sich die Vertragsparteien dazu zu respektieren, dass die streitigen Fragen auf diese Weise (und nach dem je anwendbaren Völker- oder nationalen Recht) geklärt und nicht auf diplomatischen Weg behandelt werden (Abs. 4).

\section{BITs auch im afrikanischen Raum}

Sowohl zwischen OHADA-Mitgliedern als auch im Verhältnis zu diversen anderen afrikanischen Staaten wurden um die Wende zum 21. Jahrhundert zahlreiche BITs vereinbart, die sich inhaltlich weitgehend am Stand der allgemeinen Abkommenspraxis ${ }^{78}$ ausrichten und meist dem Investor die Wahl zwischen nationaler gerichtlicher und mehreren Formen internationaler Streitbeilegung zusichern, wenn eine einvernehmliche Lösung mit dem Gastland binnen angemessener Frist nicht zustande gekommen ist (so etwa die Verträge von Benin mit Mali ${ }^{79}$ in Art. 10, mit Ghana ${ }^{80}$ in Art. 9 - beide aus 2001 - oder mit Marokko $2004^{81}$ in Art. 9). Unterschiede sind meist nur redaktioneller Art, z.B. zwischen den jeweiligen Art. 9 der BITs zwischen Burkina Faso und Tunesien (1993), ${ }^{82}$ Kamerun und Ägypten $(2000)^{83}$

76 V. 13.12.2001, https://investmentpolicy.unctad.org/international-investment-agreements/treaty-file s/446/download.

77 V. 29.6.1999, https://investmentpolicy.unctad.org/international-investment-agreements/treaty-files/ 3227/download.

78 Oben, 3.

79 V. 18.5.2001, https:/investmentpolicy.unctad.org/international-investment-agreements/treaty-files/ 5771 download.

80 V. 18.5.2001, https:/investmentpolicy.unctad.org/international-investment-agreements/treaty-files/ 442/download.

81 V. 15.6.2004, https://investmentpolicy.unctad.org/international-investment-agreements/treaty-files/ 445/download.

82 V. 7.1.1993, https://investmentpolicy.unctad.org/international-investment-agreements/treaty-files/5 604/download.

83 V. 24.10 .2000 , https://investmentpolicy.unctad.org/international-investment-agreements/treaty-file s/587/download. 
oder den Komoren und Burundi (2001). ${ }^{84}$ Bis in das zweite Jahrzehnt des 21. Jahrhunderts schlägt sich diese allgemeine Haltung auch in Musterverträgen einzelner Staaten nieder, etwa von Burkina Faso 2012. ${ }^{85}$ Dessen Art. 9 sieht sowohl als Reihenfolge zuerst Verständigung (durch Konsultationen und Verhandlungen der Streitparteien) und, nach einem Scheitern, ein Wahlrecht des Investors zwischen Gerichten des Gastlandes und Schiedsverfahren vor, wobei neben ICSID- und UNCITRAL-Mechanismen (bei ad hoc-Tribunalen) auch eine „arbitrage par la Cour Commune de Justice et d'Arbitrage (CCJA) de l'OHADA“"86 (Abs. 2 b]) eröffnet ist, als auch den Ausschluss des Einwands einer Subrogation, die abschließende und bindende Natur der Entscheidung und die Verpflichtung jeder Vertragspartei, Schiedssprüche in Übereinstimmung mit innerstaatlichem Recht durchzusetzen. Im Hinblick auf das anwendbare Recht zeigt sich schon ein gewisser Wandel, wenn es in Abs. 4 heißt: „Le Tribunal arbitral statuera sur base du droit national de la Partie Contractante, partie au différend, sur le territoire de laquelle l'investissement est situé, y compris les règles relatives aux conflits de lois, des dispositions du présent Accord, des termes des accords particuliers qui seraient conclus au sujet de l'investissement ainsi que des principes de droit international. $“ 87$

\section{Zwischenfazit: Wesentliche Sachfragen}

In der Sache geht es in BITs und auch in den einschlägigen Vorschriften anderer ,international investment agreements" (IIAs) um fünf zentrale Punkte: ${ }^{88}$ Gegenstand von Förderung und Schutz sind meist beispielhaft aufgelistete Investitionen (covered investments), wobei zuweilen im Vertrag selbst oder in einem zugehörigen Protokoll ausgeklammerte Bereiche genannt werden. In der Regel erfolgt keine Einschränkung allein auf Direkt-Investitionen, sondern werden als Kapitalanlage Vermögenswerte aller Art einbezogen. ${ }^{89}$ Andererseits wird keine förmliche Abgrenzung gegenüber kommerziellen Transaktionen ${ }^{90}$ getroffen; maßgeblich ist allein der Zusammenhang mit der Investitionstätigkeit im Gebiet des anderen Vertragsstaates, der sich zu bestimmten Arten und Formen von ,promotion“ und ,protection" verpflichtet. Dies geschieht zwar formal einem anderen Völkerrechtssubjekt, dem „home State“, gegenüber, zielt freilich auf Schaffung und vor allem Erhaltung von Rechts-

84 V. 18.5.2001, https://investmentpolicy.unctad.org/international-investment-agreements/treaty-files/ 567/download.

85 https://investmentpolicy.unctad.org/international-investment-agreements/treaty-files/2828/downlo ad.

86 https://www.ohada.org/ccja-en-bref/; näher hierzu unten, D.IV.1.

87 Dazu auch unten, bei Fn. 440.

88 Vgl. eingehend Legum (ed.), Investment Treaty Arbitration Review, 5. Aufl., 2020, Kap. 1 - 4.

89 Lehavi/Licht, Yale Journal of International Law 36 (2011), 115 (128ff.); Okpe, Afe Babalola University. Journal of Sustainable Development Law and Policy 8 (2017), 133 ff.; Lentner, ICSID Review - Foreign Investment Journal 34 (2019), 569 ff.

90 Unten, D.II.1. 
positionen von diesem (als An-/Zugehörige) zugeordneten Kapitalanlegern/Investoren (covered investors). Speziell für diese Gruppe von natürlichen oder juristischen Personen („Gesellschaften“) ist dann auch der Weg zu internationaler Streitbeilegung eröffnet, neben dem herkömmlichen über eine diplomatische oder schiedsgerichtliche Klärung von zwischenstaatlichen Meinungsverschiedenheiten. Als Investoren werden nicht nur, wenngleich meist private Unternehmen (ungeachtet der Rechtsform) erfasst, sondern auch gemischte (mit heimat- oder gaststaatlicher Kapital-Beteiligung) und „öffentliche“ Unternehmen, wenn, soweit und solange diese privatwirtschaftlich auf Märkten agieren. Gerade dann, wenn Investitionen vom und im Gastland nur in Form einer Minderheitsbeteiligung an einer dort errichteten und ansässigen Unternehmung (und gegebenenfalls zudem mit einem staatlichen Inhaber der Mehrheit der Anteile) zugelassen werden, bewirkt internationales Investitionsschutzrecht hier freilich ein „Durchstoßen des körperschaftlichen Schleiers““, ${ }^{1}$ indem auf die ausländische Beteiligung als betroffene Investition abgestellt wird. Auch bei global agierenden, „transnationalen“ Unternehmen ist kein grundsätzlich anderer Ansatz ratione personae üblich oder nötig, sondern kann auf (meist übergeordnete) Einheiten (Konzernzentralen) abgestellt werden, denen als investierende „Gesellschaft“ insoweit nach allgemeinen Kriterien ${ }^{92}$ die Nationalität des anderen Vertrags-/Heimatstaats zukommt. Räumlich erfasst werden nicht nur Landgebiete (teils auch in Übersee, wie bei Frankreich oder den Niederlanden ${ }^{93}$ ), sondern auch maritime Bereiche, vor allem Küstengewässer und ausschließliche Wirtschaftszonen nach Maßgabe von UNCLOS III. ${ }^{94}$ Ratione temporis werden BITs durchweg (meist auf zehn Jahre ${ }^{95}$ ) befristet abgeschlossen, kommt vor dem dafür maßgeblichen Ratifizierungsdatum aber bereits eine vorläufige Anwendung ${ }^{96}$ in Betracht und verlängert sich die ursprüngliche Geltungsdauer, wenn nicht rechtzeitig vor Ende der

91 „Piercing the corporate veil“; vgl. Badia, Piercing the veil of State enterprises in international arbitration, 2014.

92 (Rechtlich) Gründung oder (tatsächlich) Hauptsitz; vgl. von Hein, http://hwb-eup2009.mpipriv.de/i ndex.php/Gesellschaftsrecht,_internationales.

93 Art. 13 BIT Benin - Niederlande 2001, https://investmentpolicy.unctad.org/international-investme nt-agreements/treaty-files/446/download; Art. 12 BIT Großbritannien - Elfenbeinküste 1995, https:/investmentpolicy.unctad.org/international-investment-agreements/treaty-files/851/downloa d.

94 Art. 1 Nr. 1, 5 BIT Frankreich - Äquatorial-Guinea 1982, https://investmentpolicy.unctad.org/inter national-investment-agreements/treaty-files/1141/download; Art. 1 Nr. 1, 5 BIT Frankreich Nigeria 1990, https://investmentpolicy.unctad.org/international-investment-agreements/treaty-files /1263/download; vgl. Khamsi, ICSID Review - Foreign Investment Journal 34 (2019), 666 ff.

95 Art. 12 Abs. 2 BIT Frankreich - Algerien 1993, https://investmentpolicy.unctad.org/international-i nvestment-agreements/treaty-files/46/download; Art. 10 Abs. 1 Komoren - Mali.2001, https://inve stmentpolicy.unctad.org/international-investment-agreements/treaty-files/5772/download; BT-Drs. $18 / 4523,4 \mathrm{ff}$.

96 Dazu auch unten, Fn. 163; vgl. etwa Besserglik v. Mozambique, 28.11.2019, ICSID Case No. $\operatorname{ARB}(\mathrm{AF}) / 14 / 2, \mathrm{Rn} .325 \mathrm{ff}$. 
Laufzeit gekündigt worden ist. ${ }^{97}$ Während der Vertragsdauer (bzw. schon vorher) rechtmäßig vorgenommene Investitionen genießen regelmäßig Schutz auch nach Außerkrafttreten, für einen Zeitraum bis zu 20 Jahren. ${ }^{98}$ In deutlichem Unterschied zu der Niederlassungsund Kapitalverkehrsfreiheit im EU-Binnenmarkt ${ }^{99}$ beziehen sich Förderung und Schutz durch IIAs nicht (schon) auf den freien Zugang zum Gebiet bzw. Markt des jeweiligen Gastlandes, sondern belassen insoweit dieser Vertragspartei die Entscheidung über eine Zulassung - dem Grundsatz, der Art und Form wie dem Umfang nach. Eine hierfür etwa vorgesehene „wohlwollende“ Prüfung ${ }^{100}$ betrifft dann lediglich die Ausübung gaststaatsrechtlich eröffneter Gestaltungs- oder Ermessensspielräume. Schutz beginnt erst mit der (rechtmäßigen) Zulassung für im je relevanten Zeitraum getätigte Investitionen, für deren Bestand, aber auch daraus resultierende Erträge bis zur (autonomen) Des-Investition; das Förderungsziel erstreckt sich hingegen auch auf Maßnahmen zur Schaffung und steten Verbesserung eines „guten“ Investitions-,Klimas“101, wofür aber keine Einzelmaßnahmen skizziert oder gar vorgeschrieben werden.Seit je her kritisch ist eine klare, handhabbare Abgrenzung zwischen legitimen Rechten eines Gastlandes zur Wirtschaftsregulierung und -aufsicht ${ }^{102}$ - in fairer, nichtdiskriminierender Weise - und dessen Bestand oder zumindest (Markt-)Wert verkürzenden, gleichwohl nicht rechtswidrigen oder doch gerechtfertigten Eingriffen in Eigentumsrechte (gerade oder auch) von ausländischen Investoren. Durch in den letzten Jahrzehnten zunehmend verwendete Vorschriften soll dieser Interessenkonflikt so weit wie möglich ausgeräumt werden, was angesichts der Weite und Unbestimmtheit einschlägiger Klauseln nur teilweise gelingen kann. Beispielhaft besagt hierzu etwa Art. 11 des BIT zwischen Benin und Mauritius 2001: ${ }^{103}$ „Aucune disposition du présent Accord ne pourra être interprétée comme empêchant une Partie Contractante de prendre toute mesure nécessaire à la protection de ses intérêts essentiels en matière de sécurité, ou pour des motifs de santé publique ou de prévention des maladies affectant les animaux et les végétaux".

97 BIT UK - Elfenbeinküste 1995, Art. 14 Abs. 1; https://investmentpolicy.unctad.org/internationalinvestment-agreements/treaty-files/851/download.

9815 Jahre in Art. 12 Abs. 3 BIT Frankreich - Algerien 1993; 10 Jahre nach Art. 10 Abs. 4 BIT Komoren - Mali 2001.

99 Vgl. aber auch Mitteilung der EU-Kommission, Protection of intra-EU investment, COM(2018) $547 / 2,10 \mathrm{ff}$.

100 Vgl. schon oben, 2.

101 Vgl. nur Irtsyshcheva et al., Building favorable investment climate for economic development, 7.6.2020, http://www.growingscience.com/ac/Vol6/ac_2020_57.pdf. Zum Ziel eines ,attractive investment climate" auch Art. 1 des Trade and Investment Framework Agreement zwischen USA und ECOWAS, 5.8.2014.

102 „Right to regulate“; vgl. de Mestral/Vonhonaekker, How best to protect the right to regulate The WTO or ISA?, CIGI Papers No. 145, Sept. 2017, 2 ff.

103 V. 18.5.2001, https://investmentpolicy.unctad.org/international-investment-agreements/treaty-file s/444/download. 
Solche aus dem WTO-Recht bekannte Formulierungen (vgl. Art. XX, XXI GATT ${ }^{104}$ ) sind interpretationsbedürftig, sie belassen daher der jeweiligen Streitbeilegungsinstanz einen erheblichen Spielraum für die Entscheidung im Einzelfall, was der Rechtssicherheit kaum förderlich ist.An dieser Stelle eröffnen solche vertraglich verankerte Regulierungsvorbehalte allerdings auch die rechtliche Möglichkeit, nicht nur der anderen Abkommenspartei gegenüber, sondern generell gefasste Beschränkungen und Kontrollen im Hinblick auf ausländische Direkt-Investitionen in einzelne Wirtschaftsbranchen, vornehmlich mit Bedeutung für national wesentliche Infrastrukturen, einzurichten, wie sie aktuell (und zunehmend) auch in westlichen Industriestaaten erfolgen. ${ }^{105}$ Je nach Reichweite derartiger Klauseln bewirken diese auch eine Begrenzung der bilateral vereinbarten Meistbegünstigungsregelungen (zugunsten dritter Staaten), was eine politisch gewollte restriktive Zielrichtung gegen Unternehmen nur aus bestimmten einzelnen Ländern ${ }^{106}$ weniger angreifbar machen kann. Ob dies beabsichtigt oder sinnvoll ist, steht auf einem anderen Blatt.

\section{Neuere Regelungen und Konzepte}

\section{Kündigungen und inhaltlicher Wandel}

Schon vor dem Zusammenbruch einer sozialistisch geprägten „Zweiten Welt“, aber insbesondere seit den 1990er Jahren kam es zu einer rapiden Zunahme von IIAs zwischen außereuropäischen Staaten, auch Kontinent übergreifend. Davor hatten insbesondere einige „Ostblock"-Ländern ebenfalls mit politisch befreundeten Entwicklungsländern BITs klassischen Typs geschlossen, ${ }^{107}$ und nach dem weltpolitischen Umschwung setzten vor allem die später auch der EU beitretenden osteuropäischen Staaten diese vertragsförmige Außenwirtschaftspolitik fort. ${ }^{108}$ Ein erster Wandel zeigte sich dann Anfang des neuen Jahrtausends,

104 Dazu Mitchell (Jan. 2013), https://www.researchgate.net/publication/324167607_General_and_S ecurity_Exceptions_under_the_GATT_1994_and_the_GATS; allgemein bereits Gramlich, RiA 2020, 3 (9 ff.).

105 Vgl. unten, III.2.; für Deutschland § 4 Abs. 1 Nr. 4, 4a, § 5 Abs. 2, 3, § 14a und § 15 AWG i.d.F. von Art. 1 des Gesetzes v. 10.7.2020 (BGBl. 2020 I, 1637); dazu BT-Drs. 19/18700 v. 21.4.2020, 11 f., 18 ff.; ferner Herrmann, ZEuS 2019, 429 (432 ff.).

106 Vgl. Bierwagen/von Wistinghausen, Betriebs-Berater 2020, 1986 (1988).

107 BIT Rumänien - Senegal 1980, https://investmentpolicy.unctad.org/international-investment-agr eements/treaty-files/2207/download; mit Kamerun, https://investmentpolicy.unctad.org/internatio nal-investment-agreements/treaty-files/595/download; BIT Bulgaria - Ghana 1989, https:/invest mentpolicy.unctad.org/international-investment-agreements/treaty-files/530/download.

108 Etwa BIT Ungarn - Marokko 1991, https://investmentpolicy.unctad.org/international-investmentagreements/treaty-files/530/download, mit Ägypten 1996, https://investmentpolicy.unctad.org/int ernational-investment-agreements/treaty-files/16/download. Liste aller von EU-Staaten geschlossenen BITs in ABl. EU Nr. C 198 v. 13.6.2019, 1 ff. 
als zunächst lateinamerikanische Staaten ${ }^{109}$ und sodann auch Südafrika ${ }^{110}$ (später von Tansania gefolgt ${ }^{111}$ ) von den vorgesehenen Beendigungsklauseln ${ }^{112}$ diverser BITs Gebrauch machten und gerade nicht gewillt waren, ein „moderneres“ Anschlussabkommen zu treffen. Ähnlich wie bei einer Beendigung der Mitgliedschaft bei ICSID (nach Art. 71 des Übereinkommens ${ }^{113}$ ) hat dies allerdings keine unmittelbare Auswirkung auf schon wirksam entstandene Rechte, Ansprüche oder Verpflichtungen im jeweiligen völkervertraglichen Kontext. Dass insoweit Südafrika eine wesentliche Rolle spielt, ist wenig verwunderlich, entspricht dieser neue Ansatz doch weithin dem von zwei anderen BRICS-Staaten, ${ }^{114}$ nämlich Brasilien ${ }^{115}$ und Indien, ${ }^{116}$ und hat in der zweiten Dekade des 21. Jahrhunderts auch darüber hinaus Anhänger gefunden, über Marokko und Kolumbien (mit entsprechend aktuellen Muster-Verträgen ${ }^{117}$ ) bis hin zu Kanada, ${ }^{118}$ während andererseits Russland ${ }^{119}$ und überwiegend auch die Volksrepublik China ${ }^{120}$ bislang weitgehend an herkömmlichen Inhalten und Streitbeilegungsmechanismen festhalten. Ein Schwergewicht liegt dabei in der Einbezie-

109 Etwa Bolivien: https://investmentpolicy.unctad.org/international-investment-agreements/countrie s/24/bolivia-plurinational-state-of.. Vgl. UNCTAD, IIA Issues Note 2, Dez. 2010, $1 \mathrm{ff}$.

110 Vgl. Auflistung unter: https://investmentpolicy.unctad.org/international-investment-agreements/c ountries/195/south-africa.

111 Vgl. https://investmentpolicy.unctad.org/international-investment-agreements/countries/222/unite d-republic-of-tanzania; bisher nicht gegenüber Deutschland (s. folgende Fn.).

112 Etwa Art. 14 Abs. 2 BIT Deutschland - Tanzania v. 30.1.1965 (BGBl. 1966 II, 873); bereits oben, I.6.

113 Vgl. Tzanakopoulos, in: Hofmann/Tams (eds.), International Investment Law and General International Law, 2011, $75 \mathrm{ff}$.

114 https://infobrics.org/.

115 Ratifiziert wurden lediglich 2015 geschlossene Verträge mit Angola und Mexiko, https://investm entpolicy.unctad.org/international-investment-agreements/countries/27/brazil.

$116 \mathrm{Zu}$ diversen Kündigungen: https://investmentpolicy.unctad.org/international-investment-agreeme nts/countries/96/india.

117 Marokko 2019: https://investmentpolicy.unctad.org/international-investment-agreements/treaty-fi les/5895/download; noch weithin herkömmlich dagegen Kolumbien 2011: https://investmentpolic y.unctad.org/international-investment-agreements/treaty-files/3559/download; dazu allgemein Clodfelter, ICSID Review - Foreign Investment Law Journal, Vol. 24 (2009), 165 ff.; Brown (ed.), Commentaries on Selected Model Investment Treaties, 2013; zu Indien Hanessian/Duggel, ICSID Review - Foreign Investment Journal.32 (2017), 216 ff.

118 Model treaty 2004, https://investmentpolicy.unctad.org/international-investment-agreements/treat y-files/2820/download.

119 Vertragsmuster v. 30.9.2016, https://investmentpolicy.unctad.org/international-investment-agree ments/treaty-files/6009/download.

120 Etwa Tanzania 2013, https://investmentpolicy.unctad.org/international-investment-agreements/tre aty-files/5488/download; Mali 2009, https://investmentpolicy.unctad.org/international-investmen t-agreements/treaty-files/4923/download; hingegen zumindest betr. Streitbeilegung Konzepten der anderen Partei folgend gegenüber Kanada 2012, https://investmentpolicy.unctad.org/internati onal-investment-agreements/treaty-files/3476/download. Dazu Sheppard/Bret, 11.4.2019, https:// globalarbitrationreview.com/review/the-middle-eastern-and-african-arbitration-review/the-middl e-eastern-and-african-arbitration-review-2019/article/mining-arbitrations-in-africa. 
hung von Investorenpflichten bzw. einer ,investor responsibility“ in IIAs, die anders als Konzepte einer gesellschaftlichen Verantwortung von Unternehmen (corporate social responsibility ${ }^{121}$ ) rechtsverbindlich ausgestaltet sind; ${ }^{122}$ eine weitere inhaltliche Vertiefung in Form eines multilateralen Übereinkommens mit normativ bindenden Regelungen für transnationale Korporationen - und damit zugleich das Gros relevanter Investitionen wie Investoren erfassend - ist auf dem Wege. ${ }^{123}$ Allgemein macht sich auch im Investitionsschutzrecht der umfassende Anspruch der UN Sustainable Development Goals ${ }^{124}$ bemerkbar, etwa bei der Bewertung von Umwelt-, Gesundheits- und Beschäftigtenschutzbelangen, ${ }^{125}$ so dass eine angemessene Einordnung in den größeren Kontext eines Entwicklungsvölkerrechts ${ }^{126}$ naheliegend, wenn nicht gar notwendig erscheint. Zum gegenwärtigen Zeitpunkt ist das gewandelte ,investment policy framework ${ }^{* 127}$ allerdings (noch) allenfalls partiell in die Staatenpraxis umgesetzt, am ehesten noch in neueren nationalen Investitionsgesetzen oder -,,kodizes“ auch in einigen afrikanischen Staaten, ${ }^{128}$ und sind auf völkerrechtlicher Ebene die meisten klassischen Standards auch heute wesentlicher Vertragsinhalt. Bilaterale Abkommen neuen Typs finden sich nur vereinzelt, bei Marokko bisher allein im Verhältnis zu Nigeria, ${ }^{129}$ im Nord-Süd-Verhältnis insbesondere in BITs zwischen Kanada und (auch)

121 Vgl. Levashova, Utrecht Law Review 14 (2018), $40 \mathrm{ff}$.

122 Vgl. dazu die Beiträge in. Gathii/Puig, Symposium on Investor responsibility: The next frontier in international investment law, American Journal of International Law Unbound 113 (2019), $1 \mathrm{ff}$.; Semenko, Duties of investors in international investment law; analysis of model BITs of the latest generation, 2018, $5 \mathrm{ff}$.

123 Vgl. International Commission of Jurists, Proposal for Elements of a Legally Binding Instrument on Transational Corporations and Other Business Enterprises, Okt. 2016.

124 UNCTAD, Investment policy framework for sustainable development, 2015, 28 ff.; ferner Guiding principles for investment policymaking for the countries of the D-8 Organization of Economic Cooperation, Jan. 2020, mit dem zehn Kernprinzipien übergreifenden Ziel ,investment for sustainable development"; vgl. schon Bernasconi-Osterwalder/Johnson, International Investment Law and Sustainable Development, Key cases from 2000 - 2010, 2011; ferner UNCTAD, SDG Investment Trends Monitor, 2019; UNCTAD, The IPA Observer, Promotion investment for sustainable development in cities, Issue 7, März 2019, $1 \mathrm{ff}$.

125 Vgl. schon Ceyssens/Sekler (Fn. 48), 39 ff.; Moloo/Jacinto, Berkeley Journal of International Law 29 (2011), $1 \mathrm{ff}$.

126 Dazu bereits Schill, ZaöRV 72 (2012), $261 \mathrm{ff}$.

127 UNCTAD‘s Reform Package for the International Investment Regime, 2018, 32 ff.; UNCTAD, World Investment Report 2019, 104 ff.; UNCTAD, IIA Issues Note 1, Mai 2018, 1 ff. (Recent developments in the international investment regime); UNCTAD, IIA Issues Note 3, Juni 2019, $1 \mathrm{ff}$. (Taking stock of IIA reform: recent developments).

128 Auflistung unter: https://investmentpolicy.unctad.org/investment-laws.

129 V. 3.12.2016, https:/investmentpolicy.unctad.org/international-investment-agreements/treaty-file s/5409/download; dazu Ejims, ICSID Review - Foreign Investment Law Journal 34 (2019), 62 ff.; „klassisch“ hingegen mit Ruanda, 19.10.2016, https://investmentpolicy.unctad.org/internat ional-investment-agreements/treaty-files/5417/download. 
afrikanischen Ländern, ${ }^{130}$ während das Projekt der Niederlande ${ }^{131}$ wohl erst einmal die Frage der Grenzziehung zwischen EU- und mitgliedstaatlichen Kompetenzen lösen muss, wie sie vor allem im Rahmen von CETA (zwischen der Europäischen Union und Kanada) ${ }^{132}$ diskutiert und nicht abschließend geklärt ist. ${ }^{133}$ Welche Neuerungen dabei relevant sind oder werden, mag das Beispiel des BIT zwischen Kanada und Mali (2014) ${ }^{134}$ zeigen. Dort sind bei den „substantive provisions“ (Art. 2 - 18) herkömmliche Regeln in der Mehrzahl, freilich ergänzt durch Vorbehalte, besondere und allgemeine Ausnahmen, ein Transparenzgebot sowie eine (einzige) Vorschrift (Art. 15) zu ,health, safety, environmental measures and corporate social responsibility." Vor der Regelung zu Streitigkeiten zwischen den Vertragsparteien (Art. 37) wird in Kap. C. eine ebenso ausführliche wie eingehende eigenständige Regelung für „settlement of disputes between an investor and the host party" getroffen, die zwar weithin auch schon anderweitig vorhandene Vorgaben aufgreift, aber zudem etwa Bestimmungen über ,public access to hearings and documents“ (Art. 30) oder ,expert reports“ (Art. 33) trifft. Bemerkenswert ist zudem Anhang IV über „Exclusions from Dispute Settlement", der besagt: „A decision by Canada following a review under the Investment Canada Act is not be subject to the dispute settlement provisions under Section C (Settlement of Disputes between an Investor and the Host Party) or Section D (State-to-State Dispute Settlement Procedures) of this Agreement". Fast zur selben Zeit beinhaltet allerdings ein BIT zwischen Mali und Marokko (2014) ${ }^{135}$ fast ausschließlich herkömmliche Klauseln. Auch Marokko hat mit vielen anderen Staaten Afrikas, nicht zuletzt mit den meisten OHADA-Mitgliedern, ${ }^{136}$ am Fokus allein auf ,alte“ Standards festgehalten; erst nach Konzeption eines modernen Vertragsmuster zeigen sich in BITs mit Brasilien (2019) ${ }^{137}$ und Japan

130 Z.B. mit Guinea 2015, https://investmentpolicy.unctad.org/international-investment-agreements/t reaty-files/5095/download; mit Kamerun 2014, https://investmentpolicy.unctad.org/internationalinvestment-agreements/treaty-files/3163/download; dabei wird weithin dem Vertragsmuster von 2004 (Fn. 118) gefolgt.

131 Model Treaty v. 22.3.2019, https://investmentpolicy.unctad.org/international-investment-agreeme nts/treaty-files/5832/download; dazu auch https://www.iarbafrica.com/en/news-list/17-news/1146 -new-model-bit-negotiations-to-commence-soon 2018.

132 Comprehensive Economic and Trade Agreement, 30.10.2016, ABl. EU Nr. L 11 v. 14.1.2017, 23; zu Investitionen Kap. 8.

$133 \mathrm{Zu}$ Übergangsregeln in der EU-,,grandfathering“-Verordnung (Nr. 1019/2012 v. 12.12.2012, ABl. EU Nr. L 351 v. 20.12.2012, 40) vgl. BT-Drs. 18/7753, 13.

134 V. 28.11.2014, https://investmentpolicy.unctad.org/international-investment-agreements/treaty-fil es/3239/download.

135 V. 21.2.2014, https://investmentpolicy.unctad.org/international-investment-agreements/treaty-file s/4710/download.

136 Außer mit Mali (vorige Fn.) z.B. mit der Republik Kongo 2018, https://investmentpolicy.unctad. org/international-investment-agreements/countries/142/morocco.

137 V. 13.6.2019, https://investmentpolicy.unctad.org/international-investment-agreements/treaty-file s/5889/download. 
$(2020)^{138}$ Modifizierungen und Erweiterungen, kaum allerdings bei der im eigenen Modell ${ }^{139}$ (in Art. 28 - 43) detailliert ausgestalteten Investor-Staat-Streitbeilegung. Ein eigenes Kapitel im Vertrag mit Brasilien widmet sich zwar der „gouvernance institutionelle“ und „prévention des différends“ (Art. 14 - 19); führt dies (etwa durch Tätigwerden von Ombudspersonen, Art. 15) zu keiner Lösung, ist aber allein ein „règlement des différends“ direkt zwischen den Vertragsparteien vorgesehen (Art. 20). Im Verhältnis zu Japan handelt die ausführliche, 15 Absätze umfassende ISDS-Regelung (Art. 16) nur von gängigen internationalen Schiedsverfahren nach ICSID- oder UNCITRAL-Bestimmungen; traditionell ist auch die Beilegung von Meinungsverschiedenheiten zwischen den Vertragsparteien (in Art. 17) gestaltet. Kein BIT besteht bisher übrigens mit Kanada. Schließlich hat Südafrika bis 2010 herkömmliche BITs - ebenfalls vielfach mit afrikanischen Ländern, von Guinea bis Gabun und Senegal ${ }^{140}$ - abgeschlossen, die sich an einem 1998 erstellten Vertragsmuster ${ }^{141}$ ausrichten. Erst dann erfolgte eine Neupositionierung, zum einen 2012 im größeren Rahmen der Southern African Development Community (SADC), ${ }^{142}$ zum andern innerstaatlich durch Erlass des Protection of Investment Act 2015. ${ }^{143}$

\section{Spezifischer (regionaler) Bezug in Afrika}

Ausgeprägter als anderswo zeigt sich in Afrika die geänderte Grundhaltung oft in auf stärkere und engere (sub)regionale (wirtschaftliche) Kooperation ausgerichteten multilateralen Verträgen.

Recht frühe Reformansätze enthält der „Supplementary Act“ von 2008 zum ECOWASVertrag, ${ }^{144}$ in dem die für Mitglieder bzw. Parteien aus dieser Wirtschaftsgemeinschaft geltenden Vorschriften über „dispute settlement procedures“ (Art. 33 ff.) grundsätzlich sowohl Staat-Staat- als auch Staat-Investor- oder Investor-Staat-Streitigkeiten erfassen sollen (vor-

138 V. 8.1.2020, https://investmentpolicy.unctad.org/international-investment-agreements/treaty-files/ 5908/download.

139 Oben, Fn. 117.

140 Guinea, 25.9.2007, https://investmentpolicy.unctad.org/international-investment-agreements/treat y-files/3499/download; Gabun, 2.8.2005, https:/investmentpolicy.unctad.org/international-invest ment-agreements/treaty-files/3498/download; Senegal, 19.6.1998, https://investmentpolicy.uncta d.org/international-investment-agreements/treaty-files/5908/download.

141 https://investmentpolicy.unctad.org/international-investment-agreements/treaty-files/2845/downl oad.

142 Model BIT Template, Juli 2012; https://investmentpolicy.unctad.org/international-investment-agr eements/treaty-files/2875/download; zum Gründungsvertrag unten, Fn. 269.

143 Act No. 22 of 2015, https://investmentpolicy.unctad.org/investment-laws/laws/157/south-africa-i nvestment-act; dazu Naud et al., 11.4.2019, https://globalarbitrationreview.com/review/the-middl e-eastern-and-african-arbitration-review/the-middle-eastern-and-african-arbitration-review-2019/ article/recent-trends-in-investment-arbitration-in-africa.

144 V. 19.12.2008, https://investmentpolicy.unctad.org/international-investment-agreements/treaty-fil es/3266/download. 
behaltlich der Bereichs-Ausnahmen nach Art. 37, 38) und primär die spezifischen „Community rules" das anwendbare Recht bilden, nur subsidiär ,any other national, Community, and international law/rules agreed upon by the parties" (Art. 36). Höchst interessant ist zudem die in Art. 18 vorgenommene Verbindung von ,investor's liability“ und Streitbeilegung. So hindert etwa vor gaststaatlichen Gerichten nachgewiesene Korruption (Art. 13) eine Befassung der Streitbeilegungsinstanzen mit diesem Umstand (Art. 18 Abs. 1), und Art. 18 Abs. 6 normiert: „In accordance with the applicable domestic law, a host State or private person or organization, may initiate action for damages under the domestic law of the host member State, or the domestic law of the home member State, where such action relates to the specific conduct of the investor, for damages arising from an alleged breach of the obligation set out in this Supplementary Act. The proceedings in the domestic law court shall conform to the procedures applicable in the Community Court of Justice." Dabei geht es offensichtlich nicht um dessen (bis auf weiteres ebenfalls bestehende) Rolle als „arbitration tribunal“ nach Art. 16 des revidierten ECOWAS-Vertrags (1993), ${ }^{145}$ sondern um die zentrale richterliche Funktion des supranationalen Gerichts gemäß Art. 15, so dass die Verweisung letztlich auf die Vorschriften des einschlägigen Protokolls (nach Art. 15 Abs. 2 des Vertrags) ${ }^{146}$ abzielt. Bereits von 2006 datiert ein SADC-Protokoll „on finance and investment ${ }^{\text {" }}{ }^{147}$ In diesem Dokument selbst ist nur eine zwischenstaatliche Streitbeilegung normiert (Art. 24); jedoch gehören zum Regelwerk auch mehrere Anhänge, wovon der erste (in Ausgestaltung von Art. 3 des Protokolls) zahlreiche Vorschriften über „cooperation on investment" enthält. Angeregt wird eine Beteiligung an ,international conventions and practices" (Art. 21), wobei das ICSID- und das MIGA-Abkommen ${ }^{148}$ sowie das New Yorker Übereinkommen von $1958^{149}$ ausdrücklich genannt sind, sowie der Abschluss von BITs mit dritten Staaten (Art. 26); beides findet sich unverändert auch in der Neufassung von $2016^{150}$ (Art. 19 bzw. 24). Dies gilt auch für weitere, als Verpflichtung gefasste Vorgaben zu regionaler Kooperation (Art. 22/20), mitgliedstaatliche ,investment promotion agencies" (Art. 23/21) und Beziehungen zu anderen regionalen und internationalen Organisationen in Investitionsfragen (Art. 25/23). Art. 25 (früher Art. 27) hält SADC-Mitglieder

145 V. 24.7.1993, https://investmentpolicy.unctad.org/international-investment-agreements/treaty-file s/5476/download.

146 http://prod.courtecowas.org/wp-content/uploads/2018/11/Protocol_AP1791_ENG.pdf; http://prod .courtecowas.org/wp-content/uploads/2018/11/Supplementary_Protocol_ASP.10105_ENG.pdf.

147 https://www.sadc.int/files/4213/5332/6872/Protocol_on_Finance_Investment2006.pdf; zur Originalfassung 2007 Muchlinski, The COMESA Common Investment Area: Substantive Standards and Procedural Problems in Dispute Settlement, SAOS Research Paper No. 11/2010, 1 ff.

148 Convention establishing the Multilateral Investment Guarantee Agency v. 11.10.1985, https://ww w.miga.org/sites/default/files/archive/Documents/MIGA\%20Convention\%20(April \%202018).pdf.

149 V. 10.6.1958, http://www.newyorkconvention.org/11165/web/files/original/1/5/15457.pdf; dazu bereits Gramlich, RiA 2020, 3 (24).

150 https://www.sadc.int/files/7114/9500/6315/Agreement_Amending_Annex_1__CCooperation_on_ investment_-_on_the_Protocol_on_Finance_Investment_-_English_-_2016.p $\overline{\mathrm{df}}$. 
dazu an, zu gewährleisten, ,that investors have the right of access to the courts, judicial and administrative tribunals, and other authorities competent under the laws of the host State for redress of their grievances in relation to any matter concerning their investment." Für Investor-Staat-Streitbeilegung sah die Erstfassung des Anhangs in Art. 28 noch vor, die beiden Parteien könnten sich insoweit entweder auf das SADC Tribunal, ${ }^{151}$ ein ICSID- oder ein ad hoc-Schiedsverfahren nach UNCITRAL-Regeln verständigen; die revidierte Nachfolgeregelung in Art. 26 kennt hingegen nur eine Staat-Staat-Streitbeilegung im Rahmen des Protokolls über das Tribunal. Das Investment Agreement für die COMESA Investment Area von $2007^{152}$ hält zunächst die Mitglieder an, an ,international multilateral agreements" mitzuwirken, Art. 6 nennt außer den drei auch im SADC-Protokoll aufgeführten noch das Agreement establishing the African Trade Insurance Agency ${ }^{153}$ sowie jeden weiteren mehrseitigen Vertrag ,designed to promote or protect investment" (definiert in Art. 1 Nr.9). Teil III über „,dispute settlement“ behandelt nach „negotation and mediation“ (Art. 26, auch im Investor-Staat-Verhältnis) sowie zwischenstaatlichen Meinungsverschiedenheiten (Art. 27) in Art. 28 ,investor-State disputes“ und eröffnet dabei dem Unternehmen nach Scheitern einvernehmlicher Lösung die Wahl zwischen ,the competent court of the member State in whose territory the investment has been made", „the COMESA Court of Justice“ (gemäß Art. 28 [b] des COMESA-Vertrags ${ }^{154}$ ) oder mehreren Formen von ,international arbitration"; nur wenn weder nach ICSID- (einschließlich der Additional Facility Rules) oder nach UNCITRAL-Regeln verfahren wird, bedarf es dazu einer Vereinbarung mit der (gast)staatlichen Seite. Neben einigen konkreten prozeduralen Vorschriften (etwa zu amicus curiae-Eingaben ${ }^{155}$ ) wird explizit die Wahl eines bestimmten Forums als endgültig erachtet, dem Investor also die Einschaltung einer weiteren Stelle ,relating to the same subject matter or underlying measure" verboten. Im Hinblick auf die Demokratische Republik Kongo und die Komoren erlangt dies spezielle Bedeutung wegen deren gleichzeitiger Zugehörigkeit zum OHADA-Raum (und bezüglich des dort eingerichteten CCJA ${ }^{156}$ ). Für alle Schiedsverfahren des Teils III gelten Vorschriften zur ,enforceability of final awards" nach mitgliedstaatlichem Recht (Art. 29), über eine vom COMESA-Sekretariat zu führende Liste von qualifizierten ,arbitrators“ (Art.30) und schließlich zum ,governing law in disputes"; gemäß Art. 31 entscheidet das Schiedsgericht ,in accordance with this Agreement, the COMESA treaty, national law of the host State, and the general principles of international law". Eine (noch nicht in Kraft getretene) revidierte und erweiterte Fassung

151 https://www.sadc.int/about-sadc/sadc-institutions/tribunal/; s. auch unten, Fn. 480.

$152 \mathrm{https} / / /$ investmentpolicy.unctad.org/international-investment-agreements/treaty-files/3092/downl oad.

153 http://www.ati-aca.org/.

154 V. 5. 11.1993, https:/www.comesa.int/wp-content/uploads/2020/07/Comesa-Treaty.pdf.

155 Unten, Fn. 394.

156 Näher unten, D.IV.1. 
des Abkommens ${ }^{157}$ soll einer Anpassung an den von der Afrikanischen Union vorangetriebenen Pan African Investment Code ${ }^{158}$ dienen und damit letztlich auch an ein ,investment

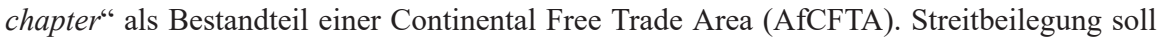
dann bevorzugt auf nationaler und regionaler Ebene erfolgen. Dies ähnelt weitgehend dem Mustervertrag der East African Community (EAC) von 2016, ${ }^{159}$ wo zu Art. 23 zunächst verlautet, die ,preferred option“ sei „,not to include investor-State dispute settlement", sodann aber ein ausführliches Regelungsmodell enthalten ist, in dem als ,applicable arbitration rules" außer denen von ICSID und UNCITRAL auch eine Befassung des EAC Court of Justice vorgesehen wird (anknüpfend an dessen Kompetenz nach Art. 32 EAC-Vertrag ${ }^{160}$ ).

\section{Aushandlung und Umfeld von internationalen Investitionsabkommen}

Anscheinend werden zahlreiche BITs und IIAs zwar nach Aushandlung der Texte unterzeichnet, die im Hinblick auf ihre Inhalte regelmäßig vor einer Ratifizierung (durch das Staatsoberhaupt) und das endgültige Inkrafttreten gebotene parlamentarische Billigung (in Gesetzesform) ${ }^{161}$ kommt aber nicht bei allen Parteien zustande; ${ }^{162}$ gleichwohl kann hier dann vorübergehend eine vorläufige Anwendung ${ }^{163}$ vereinbart sein und stattfinden. Da der Volksvertretung allerdings nach Paraphierung von Abkommensdokumenten nur noch eine Wahl zwischen Zustimmung oder Ablehnung möglich ist, geht der internationale Trend hier in Richtung frühzeitiger Einbeziehung von Parlamentariern bereits in den Aushandlungsprozess, was zugleich diesen eröffnete hinreichende Information über den aktuellen Stand einschließlich noch offener Punkte voraussetzt und damit ein Mindestmaß an Transparenz. ${ }^{164}$ Insbesondere im vergangenen Jahrzehnt haben Regierungen auch eine angemessene Beteiligung der „Zivilgesellschaft“ bzw. von Nichtregierungsorganisationen (NGOs) bei Freihandels- und Investitionsabkommen als „wichtiges Anliegen“ gekennzeichnet, ${ }^{165}$ um sachlich gute und zugleich weithin akzeptable Lösungen zu finden. Ähnlichen Zwecken dienen die Einrichtung von „consultative committees“ in Economic Partnership Agreements

157 https://www.comesa.int/plans-afoot-to-publicize-common-investment-area-agreement/.

158 Dazu bereits Gramlich, RiA 2020, 3 (15 f.).

159 https://www.eac.int/documents/category/investment-promotion-private-sector-development; vgl. schon Gramlich, RiA 2020, 3 (18f.).

160 https:/www.eacj.org//wp-content/uploads/2012/08/EACJ-Treaty.pdf.

161 Vgl. dazu unten, C.III.1.b).

162 Details im International Investment Agreements Navigator, https://investmentpolicy.unctad.org/.

163 Vgl. oben, bei Fn. 96; zu CETA BT-Drs. 18/8583 v. 30.5.2016, 4 f. (Bundesregierung, Probleme der vorläufigen Anwendung von CETA).

164 Zur Transparenz und „confidentiality“ bei Streitbeilegungsverfahren unten, Fn. 396; allgemein anhand der TIPP-Diskussion Bundesregierung, BT-Drs. 18/7299 v. 29.1.2016, $1 \mathrm{ff}$.

165 Vgl. Bundesregierung, Die Beschlüsse von Bali, bilaterale Handels- und Investitionsschutzabkommen und die Auswirkungen auf Entwicklungsländer, BT-Drs. 18/632 v. 20.2.2014, 8 . 
(EPA), ${ }^{166}$ die AKP-EU-Follow-Up Committees ${ }^{167}$ oder die Schaffung eines ,enabling environment“ auch für die ,civil society“ in Art. 127 EAC-Vertrag. ${ }^{168}$ Angesichts der Langzeitwirkungen von IIAs ist auch die Institutionalisierung einer permanenten „Begleitung“ eines Vertrags und dessen Durchführung in der Praxis als Instrument flexibler Anpassung an sich ändernde externe und interne Verhältnisse immer wichtiger geworden und wird daher insbesondere in neuere Freihandelsabkommen der EU (deep and comprehensive free trade agreements) ${ }^{169}$ regelmäßig aufgenommen, auch in CETA (Art. 26.1. - Joint Committee). In BITs scheinen sich generelle Konsultationsklauseln ebenfalls zunehmend zu verfestigen, wie das Abkommen zwischen Marokko und Brasilien ${ }^{170}$ zeigt. Dort wird zudem auch ein Bezug zu „nationalen Kontaktpunkten“ normiert, als zentrale Anlaufstelle für die Unterstützung ausländischer Investoren im Gastland, und damit ein von der OECD ${ }^{171}$ vorangetriebener prozeduraler Ansatz einer Konfliktprävention genutzt. ${ }^{172}$

\section{Verfeinerungen durch Richterrecht?}

Verschiedene Themen und Konzepte neuerer IIAs sind auch bereits in Schiedssprüche eingeflossen, weniger im Hinblick auf modernisierte oder reformierte Vorschriften in solchen Abkommen als durch eine ,progressive“, durch die Vorgaben zum je anwendbaren Recht aber durchaus abgedeckte arbitrale Rechtsprechung oder auch Rechtsfortbildung. ${ }^{173}$ Freilich bleibt diese diffus, weil es insoweit weder hinreichende personelle noch institutionelle Verschränkungen gibt und Einzelfallentscheidungen jedenfalls nur weitaus schwächer als in hierarchischen und zentralisierten Justizstrukturen Präzedenzwirkung entfalten können. Immerhin umfassen einschlägige „awards“ ein recht breites Spektrum, von Anwendungsbereich (sowie Ausnahmen vor allem im Steuersektor) und Definitionen der Abkommen über

166 Etwa zur CARIFORUM-EU-Wirtschaftspartnerschaft Art. 232 des Abkommens v. 15.9.2008; dazu BT-Drs. 18/8297 v. 2.5.2006, 77; https://www.eesc.europa.eu/en/sections-other-bodies/other/c ariforum-eu-consultative-committee; ferner Bundesregierung, Neuverhandlungen von Handelsabkommen mit Afrika, BT-Drs. 18/13393 v. 24.6.2017, 1 ff. Das EPA zwischen EU und deren Mitgliedstaaten einer-, ECOWAS und UEMOA (Westafrika“") andererseits sieht sowohl einen Paritätischen Beratungsausschuss (Art. 97) als auch einen Gemeinsamen Parlamentarischen Ausschuss (Art. 96) vor (Rat der EU, Dok. Nr. 13370/14, 2.12.2014).

167 https:/www.eesc.europa.eu/en/sections-other-bodies/other/eu-acp-follow-committee.

168 Oben, Fn. 160

169 Vgl. schon Bundesregierung, Private Schiedsgerichte in Freihandelsabkommen, BT-Drs. 18/6315 v. 13.10.2015, $2 \mathrm{ff}$.

170 Oben, Fn. 137, insbesondere Art. 14; zu ,ombudsmen“ aaO, Art. 15.

171 Vgl. Teil II der OECD Guidelines for Multinational Enterprises, 2011, http://www.oecd.org/daf/i nv/mne/48004323.pdf.

$172 \mathrm{Zu}$, ,contact points“ auch Art. 26.5 CETA; dazu ferner unten, D.V.

173 Vgl. UNCTAD, IIA Issues Note No. 4 (Juli 2019), 1 ff. (Review of ISDS decisions in 2018: Selected IIA reform issues); als Bsp. etwa Cortec Mining et al. v. Kenya, ICSID Case No. ARB/15/29, 22.10.2018, https://www.italaw.com/sites/default/files/case-documents/italaw10051. pdf. Ein Annullierungsantrag ist anhängig. 
wesentliche Maßstäbe zu Behandlung und Schutz (wie Nichtdiskriminierung, FET oder „expropriation“174), die Reichweite von „umbrella clauses“ bis zu Begrenzungen durch „public policy“/“ordre public“ und Notstand (necessity defense ${ }^{175}$ ). Gleiches gilt für prozedurale Fragen unter Einschluss (von Optionen) des Zugangs zur internationalen Streitbeilegung nach dem ISDS-Schema.

\section{Nationales Investitionsrecht}

\section{Bezüge zur internationalen Ebene}

Regelungen über Zulassung und Behandlung von Investitionen im Inland auch (oder speziell) durch Investoren aus anderen Staaten mögen sich schon im Hinblick auf die Situation beim Gesetzeserlass in diversen Rechtsakten finden, in Bundesstaaten zumeist auf der zentralstaatlichen Ebene. Nicht nur, aber überwiegend in afrikanischen wie anderen Entwicklungsländern wird hier allerdings oft die Form einer Kodifikation in einem einzigen Regelwerk gewählt, weil die dadurch erreichte Übersichtlichkeit - vor allem wenn sie durch ähnlich straffe und kompakte Vollzugsorganisation komplettiert wird - bereits einen wichtigen Anreiz für die Vornahme von Kapitalanlagen und die damit verbundenen zahlreichen Einzelvorgänge bildet. ${ }^{176}$ Zudem dienen solche Kodizes auch der Ergänzung lückenhafter oder als Ersatz fehlender (oder nicht mehr bestehender) völkerrechtlicher Förderungs- und Schutz-Verpflichtungen und zugleich als normative Basis, mit Investoren wirksame Schiedsklauseln durch Vertrag nach innerstaatlichem Recht (contract) ${ }^{177} \mathrm{zu}$ vereinbaren. Wegen der Form als (verfassungsgemäßes) Gesetz ist überdies durchweg eine ausreichende Legitimation durch das Parlament gewährleistet. Im OHADA-Raum sind insoweit etwa ,investment codes" von Niger oder Togo ${ }^{178}$ zu nennen, ein anderes Beispiel ist die tansanische Regelung von 1997. ${ }^{179}$ Speziell den je aktuellen ,investment policies“ einzelner Staaten widmet sich die UNCTAD im Rahmen von ,reviews“ ${ }^{180}$ So haben von dieser UN-Ein-

174 Vgl. den Bericht über die Ergebnisse der öffentlichen Online-Konsultation zu TTIP, Commission Staff Working Document, SWD (2015) 3 final v. 13.1.2015, $16 \mathrm{ff}$.

175 Vgl. Alvarez/Khamsi, The Argentine crisis and foreign investors, IILJ Working Paper 2008/5, 15 ff., 43 ff.; Franke, in: Hofmann/Tams (Fn. 113), 121 ff.; BVerfGE 118, 124 (134 ff.).

176 Als wesentlicher Teil eines günstigen Investitionsklimas; so auch UNCTAD, World Investment Report 2019, 86 ff.

177 Vgl. Crawford, Arbitration International 24 (2008), 351 ff.; Wackernagel, Das Verhältnis von treaty und contract claims in der internationalen Schiedsgerichtsbarkeit, 2009, $5 \mathrm{ff}$.

$178 \mathrm{Zu}$ Togo (Loi No. 2012-001) https://investmentpolicy.unctad.org/investment-laws/laws/148/togo -investment-code; zu Niger (Loi No. 2014-09) https://investmentpolicy.unctad.org/investment-la ws/laws/206/niger-code-des-investissements-.

179 Act No. 26 of 1997, https://investmentpolicy.unctad.org/investment-laws/laws/60/tanzania-united -republic-of-investment-act.

$180 \mathrm{https} / /$ investmentpolicy.unctad.org/investment-policy-review. 
richtung beauftragte Experten für die Elfenbeinküste ${ }^{181}$ jüngst nicht nur den ,rechtlichen Rahmen" für Investitionen (außer speziellen Vorschriften für ausländische auch allgemeine, von der Unternehmensgründung bis zu „corporate governance“) analysiert, sondern auch ausländische Direktinvestitionen, Wettbewerbsfähigkeit und regionale Entwicklung näher beleuchtet und empfohlen, die Förderung besser zu koordinieren und dabei strategisch und proaktiv vorzugehen, insbesondere durch Stärkung der Rolle des Centre de Promotion des Investissements (CEPICI). ${ }^{182}$

\section{Investitions-Lenkung durch Förderung und Kontrolle}

Investment Promotion Agencies (IPAs) ${ }^{183}$ sind freilich ebenfalls keine nur in Entwicklungsländern anzutreffenden staatlichen oder staatsnahen Institutionen, sondern auch in OECDStaaten regelmäßig vorhanden, dort allerdings nicht speziell auf Anbahnung von Kontakten und Pflege des Umgangs mit ausländischen Investoren ausgerichtet. ${ }^{184}$ Viele Industrieländer (und auch die EU) betrachten allerdings angesichts weiter wachsender Globalisierung einzelne ausländische Investoren/Gesellschaften im Hinblick auf deren Herkunftsstaaten als zumindest überwachungsbedürftig und nutzen offenbar zunehmend ein (oft bisher zwar existentes, aber nur auf dem Papier stehendes) Arsenal von Eingriffsmaßnahmen zur Verhinderung von Übernahmen oder anderen spürbaren Einflussnahmen auf (bisher) inländische Unternehmen, deren Tätigkeiten für eine (im weiten, nicht nur genuin politische Aspekte umfassenden Sinne verstandene) nationale Sicherheit (nach außen wie im Innern) für wichtig erachtet werden. ${ }^{185}$ Solche Interventionen und Restriktionen auslösende Umstände lassen sich nicht nur aus Erwägungen zur Daseinsvorsorge für die eigene Bevölkerung begründen, sondern auch aus der Überlegung heraus, technische oder gesamtwirtschaftliche Infrastrukturen könnten vom Ausland her (aus politisch suspekten Staaten) manipuliert oder anders beeinträchtigt werden. ${ }^{186}$ Ein derart genereller Kontrollansatz birgt allerdings die Gefahr einer Rechtszersplitterung, da die Definition von „,national security“ letztlich selbst

181 2019, https://unctad.org/system/files/official-document/diaepcb2019d5_fr.pdf.

$182 \mathrm{AaO}$ (vorige Fn.), 9 ff., 47 ff. und insbes. 73 ff.

183 S. bereits oben, I.2.; aktuell UNCTAD, IPA Observer, Special Issue 9, Juli 2020 (PostCOVID-19: Investment promotion agencies and the ,new normal“).

184 Krit. dazu Drillisch/Sekler, Bilaterale Investitionsabkommen und Investitionsgarantien, 2004, $20 \mathrm{ff}$.

185 Vgl. etwa Gerhardt, Wirtschaftsdienst 2018, 814 ff.; Verordnung (EU) 2019/452 v. 19.3.2019, AB1. EU Nr. L 79I v. 21.3.2019, 1; allgemein UNCTAD. Investment Policy Monitor, Special Issue, Dez. 2019 (National security-related screening mechanisms for foreign investment); UNCTAD, World Investment Report 2019, 85 f., 89 ff.

186 Vgl. Mitteilung der EU-Kommission, Welcoming Foreign Direct Investment While Protecting Essential Interests, $\operatorname{COM}(2017) 494$ final v. 13.9.2017; Commission Staff Working Document, Foreign direct investment in the EU, SWD(2019) 108 final, 13.3.2019, 8 ff.; Bericht der Kommission über Handels- und Investitionshindernisse 2019, COM(2020) 236 final v. 15.6.2020, $14 \mathrm{f}$. 
in einem Staatenverbund wie der EU jedem einzelnen Staat überlassen ist und gerade nicht zum Gegenstand einer schiedsgerichtlichen Klärung gemacht werden kann. ${ }^{187}$

\section{Zwischenergebnis}

Klassisches wie modernes internationales Investitionsschutzrecht hat mithin auf völkerrechtlicher Ebene für vielfältige Abschnitte im Lebenszyklus einer Investition im Ausland Rahmenvorgaben getroffen, die trotz der je meist nur einzelne Vertragsparteien untereinander verpflichtenden Struktur der relevanten Rechtsquellen zwar weiter verbessert werden können und sollten, zu denen aber auch keine wirkliche Alternative ersichtlich ist. Eine entsprechend inhaltliche Vereinheitlichung des (Markt-)Zugangs ausländischen Kapitals kann - anders als die letztlich auch grundrechtlich verankerte (Eigentums-)Bestands- und Wertgarantie von schon getätigten Investitionen - jedenfalls derzeit nicht über eine räumliche Erweiterung von unternehmerischer Entfaltungs- oder allgemeiner Berufsfreiheit legitimiert werden, da diese auf internationaler Ebene gerade nicht in gleicher Weise verbürgt ist, vielmehr häufig durch Verfassungsrecht nur oder in besonderer Weise den je eigenen Staatsangehörigen gewährleistet wird. ${ }^{188}$ Bei Streitigkeiten zwischen (ausländischem) Investor und Gastland ist zwar die Festlegung des dabei maßgeblichen anwendbaren Rechts eine ganz zentrale Frage, aber nicht weniger auch (und dem vorausgehend) die Entscheidung darüber, wer überhaupt zu einer verbindlichen und endgültigen Streitbeilegung berufen ist. Wenn

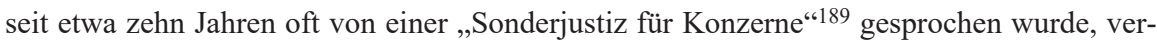
birgt sich hinter der polemischen Zuspitzung ein durchaus reales Problem, nämlich die Bestimmung von Reichweite und Inhalt des von jedem Staat infolge seiner existenziellen Befriedungsfunktion zu erfüllenden Justizgewährleistungsanspruchs, den ein partieller oder ganz gänzlicher Verweis auf alternative, nicht-gerichtliche Streitbeilegung notwendig verkürzt oder gar obsolet macht, wenn und solange diese nicht funktionell gleichartig und gleichwertig eingerichtet wird. Hierauf soll, ja, muss im Folgenden spezielles Augenmerk gelegt werden.

187 Ähnlich wie bei Art. XXI GATT; dazu Wang. Chinese Journal of International Law 18 (2019), 695 ff.; Voon, American Journal of International Law Unbound 113 (2019), 45 ff.

188 Etwa Art. 12 i.V.m. Art. 116 Abs. 1 und Art. 19 Abs. 3 GG.

189 Vgl. nur BT-Drs. 18/6818 v. 25.11.2015, 1 ff. (Antrag DIE LINKE, Für eine lebendige Demokratie - Fairer Handel statt TTIP und CETA); BT-Drs. 18/1457 v. 21.5.2014, 2 (ANTRAG BÜNDNIS 90/DIE GRÜNEN, Für ein starkes Primat der Politk). 


\section{Mechanismen zur Beilegung internationaler wirtschaftlicher Streitigkeiten mit Beteiligten in/aus mindestens zwei Staaten}

\section{Allgemeines}

\section{Grundlagen}

„Investment disputes“ bilden einerseits einen Ausschnitt aus dem größeren Bereich wirtschaftlicher Meinungsverschiedenheiten (im Unterschied zu rein privaten oder familiären), lassen sich zum andern jedoch von „commercial transactions" dadurch unterscheiden, dass hierbei nicht Waren-, Dienstleistungs- oder Personenverkehr im Mittelpunkt stehen, sondern Kapital-Anlagen (auch in Form von Niederlassungen) und damit verbundene (finanzielle) Transaktionen, auch wenn die Investorentätigkeit oft auch den Einsatz von Personal und Sachkapital mit sich bringen wird. ${ }^{190}$ Zwar können ferner bei Handelssachen auch staatliche bzw. öffentliche Unternehmen direkt als betroffene Vertragspartei beteiligt sein, jedoch wirken hier hoheitliche Maßnahmen unmittelbar als (störender) Faktor auf die Geschäftsbeziehung ein, indem deren Abwicklung rechtlich oder tatsächlich erheblich erschwert oder gar verunmöglicht wird; die Interventionen selbst sind hingegen nicht direkt Gegenstand des (Rechts-)Streits. Bei Behandlung oder Schutz von Investitionen ist demgegenüber das vertikale Verhältnis Investor/Unternehmen und (Gast-)Staat maßgeblich, nicht nur, wenn die staatliche Seite den für sie geltenden normativen Anforderungen nicht entspricht, sondern auch, wenn der Investor seinerseits völkerrechtlich und vor allem innerstaatlich normierten Verpflichtungen nicht gehörig nachkommt. „International“ ist (auch) eine Investitionsstreitigkeit nicht im Hinblick auf das für die rechtliche Bereinigung anwendbare (Völker-)Recht, maßgeblich für solche Einordnung sind vielmehr tatsächliche Anknüpfungspunkte, insbesondere dass an der Auseinandersetzung private oder auch gouvernementale Akteure aus mehr als einem staatlichen Territorium und/oder unterschiedlicher Staats an- bzw. -zugehörigkeit beteiligt sind. ${ }^{191}$ Je nach Status und Struktur der Streitparteien handelt es sich dann, wie dies der Blick auf zahlreiche IIAs zeigt, entweder um Staaten-Staaten- oder um Investor-Staat-Streitigkeiten, wobei bei jenen auch andere Völkerrechtssubjekte (wie die EU oder weitere Regional Economic Communities, $\mathrm{RECs}^{192}$ ) als Partei auftreten können und bei diesen Investoren nicht nur als Kläger agieren, sondern auch zumindest einer Widerklage der (gast)staatlichen Seite ausgesetzt sein können. ${ }^{193}$ Schließlich können Streitbeilegungseinrichtungen und -verfahren zum einen mittels eines

190 Daher Regelungen etwa zu „senior management, board of directors, and entry of personnel“; z.B. Art. 3 BIT Kanada - Guinea, https://investmentpolicy.unctad.org/international-investment-a greements/treaty-files/5095/download.

191 Zu anerkannten Anknüpfungspunkten vgl. Tanaka, The Peaceful Settlement of International Disputes, 2018, 8 ff.; BGH, 22.11.2016, II ZB 19/15, Der Betrieb 2017, 479 (480 f.); ferner Société civile immobilière de Gaeta v. Guinea, 21.15.2015, ICSID Case No. ARB 12/36, Rn. 106 ff.

192 In Afrika etwa SADC, COMESA oder OHADA.

193 Unten, Fn. 391. 
multi- oder bilateralen völkerrechtlichen Vertrags eingerichtet und ausgestaltet werden (intergouvernemental), aber auch auf (Organisations-)Verträgen nationalen Rechts (des jeweiligen Hauptsitzes) beruhen, was freilich kein Hindernis dafür bildet, dass bei solch' privat geregelter Streitbeilegung öffentliche Unternehmen oder selbst Staaten als solche auf der einen oder anderen Seite beteiligt sind. ${ }^{194}$ Ein dritter, hybrider Ansatz bedient sich eines speziellen staatlichen (bzw. gemeinschaftlichen) Rechtsaktes, durch den entweder innerhalb der normalen Gerichtsbarkeit Stellen geschaffen werden, die nicht nur als Justiz-, sondern auch als Schiedsorgane tätig werden dürfen, ${ }^{195}$ oder aber besondere Einrichtungen/Zentren gerade für internationale Mediation, Schlichtung oder „,arbitration“ (im Hinblick auf Handels- oder auch Investitionsangelegenheiten) eine auf sie zugeschnittene gesetzliche Grundlage erhalten. ${ }^{196}$ Kompetenzüberschneidungen sind dabei kaum vermeidbar, auch auf intergouvernemental-globaler Ebene, weil sich Streitgegenstand und Entscheidungsmaßstab oft überlagern. Eine klare Trennung ist hier wohl nur gegenüber ausschließlich zwischen Völkerrechtssubjekten als Hauptparteien durchgeführten dispute settlement-Verfahren möglich, wie im Rahmen der WTO; ${ }^{197}$ werden dort vor einem Panel oder dem Appellate Body Fragen der richtigen Auslegung von Vorschrift des GATS ${ }^{198}$ oder auch des TRIMs-Abkommens ${ }^{199}$ geklärt, so hat dies auch bei inhaltlichem Bezug zum Investitionsrecht jedenfalls nur argumentativ Bedeutung für ISDS-Verfahren, selbst wenn ein Staat Partei sowohl aller relevanten Übereinkommens als auch des WTO-Verfahrens war, in dem eine konkrete Interpretation erfolgte.

\section{Nicht-staatliche versus gouvernementale Streitparteien - ISDS}

Die Schaffung und Entwicklung des ISDS-Konzepts in der zweiten Hälfte des 20. Jahrhunderts hat nicht dazu geführt, dass bereits früher entstandene Regularien für eine intergouvernementale Beilegung von Meinungsverschiedenheiten nur und direkt zwischen Staaten ihre Bedeutung verloren haben, vielmehr werden diese fast durchweg in IIAs neben ISDS eigens genannt ${ }^{200}$ und reichen zudem in ihrem Anwendungsbereich weit über Investitions-

194 Bsp. Vattenfall AB v. Deutschland (2), ICSID Case No. ARB/12/12; dazu https://www.bmwi.de/ Redaktion/DE/Artikel/Energie/vattenfall-gegen-bundesrepublik-deutschland.html.

195 Nicht zuletzt in Afrika; unten, D.IV.; aber auch Güteverfahren vor Zivilrichtern in Deutschland nach $\S 278$ (insbes. Abs. 5) ZPO.

196 Näher unten, III.4.

197 Vgl. nur Tanaka (Fn. 191), 275 ff.

198 General Agreement on Services and Trade, Anhang I B zum WTO-Abkommen v. 15.4.1994, https://www.wto.org/english/docs_e/legal_e/26-gats_01_e.htm.

199 Agreement on Trade-Related Investment Measures, Anhang I A zum WTO-Abkommen, https:// www.wto.org/english/docs_e/legal_e/18-trims_e.htm.

200 Z.B. Art. 10, 11 BIT Deutschland - Madagaskar v. 1.8.2006 (BGB1. 2008 II, 478); Art. 8, 11 BIT Frankreich - Guinea, 26.7.2007, https://investmentpolicy.unctad.org/international-investment-agr eements/treaty-files/3397/download. 
schutzrecht hinaus. ${ }^{201}$ Gemeinsam ist dabei nicht nur die institutionelle Basis im Völker(vertrags)recht, sondern auch ein Streitgegenstand öffentlich-rechtlicher Natur mit Konsequenzen für das der Entscheidungsfindung zugrunde zu legende, „anwendbare“ Recht, das auch staatlichen Vertragsparteien Grenzen setzt. Dazu zählen zunächst die je vertraglich festgelegten Regelungen/Verhaltensstandards ${ }^{202}$ und zu deren Ergänzung oder auch Konkretisierung Normen aus anderen Völkerrechtsquellen, soweit diese allgemein (als Gewohnheitsrecht oder Rechtsgrundsatz) anerkannt sind oder aber Vertragsklauseln überlagerndes zwingendes Recht (ius cogens) darstellen, was zumindest einen Kern von Menschenrechten mit einschließt. ${ }^{203}$ Private Handelsschiedsgerichtsbarkeit hingegen vollzieht sich auch bei Mitwirkung staatlicher Parteien auf der Grundlage einer weithin freien Wahl nationalen privaten oder speziellen Handels-Rechts; selbst wenn einzelne Vorschriften einer internationalen lex mercatoria ${ }^{204}$ herangezogen werden (dürfen), wären diese ebenfalls Ergebnisse einer (legitimen) ,privaten Rechtsetzung“. Freilich ist der durch das anzuwendende Recht vorgegebene Prüfungsmaßstab in ISDS-Verfahren viel weniger detailliert und konkret als der normative Rahmen für (Vertrags-)Pflichterfüllung in nationalem Zivilrecht, selbst dann, wenn in BITs oder anderen Abkommen explizit auch die Einhaltung von Verpflichtungen zugesagt (und deren Rechtsverletzung als relevanter Vertragsverstoß behandelt) wird, welche sich erst aus dem anlässlich eines auf eine konkrete Investition eines ganz bestimmten Investors bezogenen „,contract“ (als Äquivalent einer gesetzlichen oder behördlichen Zulassung) ${ }^{205}$ mit dem Gaststaat ergeben (umbrella clause ${ }^{206}$ ). Ist also erst einmal ein förmliches außergerichtliches Verfahren in die Wege geleitet, so resultiert aus dem defizitären bzw. unscharfen Prüfungsmaßstab notwendig eine erhöhte Bedeutung der Entscheider-Persönlichkeit (ungeachtet der auch hier vorhandenen prozeduralen, oft justizähnlichen Regeln), nicht nur im Hinblick auf fachliche Qualifikation, sondern zudem auf eine Vielzahl eher „weicher“ Faktoren wirtschaftlicher wie kultureller Art, die erheblich vom Regelstatus eines staatlichen Richters abweichen. ${ }^{207}$ Selbst eine institutionelle oder prozedurale Konsolidierung wie bei ICSID bzw. UNCITRAL bewirkt nur ansatzweise oder punktuell die Herausbildung einer „ständigen Rechtsprechung“ über die jeweilige Einrichtung hinaus, zumal die personelle Zusammensetzung von Entscheidungsgremien sich (der Konstruktion geschuldet) laufend ändert. Harmonisierung, Vereinheitlichung oder gar Rechtsfortbildung über Mechanismen einer Justizhierarchie scheiden aus, Präjudizien mögen zwar mehr oder weniger häufiger gesucht (wieder je nach Persönlichkeit, Einsatzspek-

201 Generell Tanaka (Fn. 191), 105 ff.

202 Dazu bereits oben, B.

203 Vgl. Geldermann, Völkerrechtliche Pflichten multinationaler Unternehmen, 2009, $307 \mathrm{ff}$.

204 Vgl. Lehavi/Licht, Yale Journal of International Law 36 (2011), 115 (149 f.).

205 Zum Zweck einer „Stabilisierung“ geltenden Rechts Cotula, Foreign investment contracts, $2 \mathrm{f}$., https://pubs.iied.org/pdfs/17015IIED.pdf.

206 Vgl. schon oben, bei Fn. 41; zur Verknüpfung näher Wackernagel (Fn. 177), 15 ff.

207 Unten, C.IV.2.b). 
trum und Herkunft des Entscheiders) und als Begründungselemente herangezogen werden ("Homogenitätssicherung"208), aber das geschieht lediglich ad hoc und ohne rechtliche Notwendigkeit. ${ }^{209}$ Schon strukturell kommt schließlich ein ISDS-Verfahren erst dann ernsthaft in Betracht, wenn der angeblich rechtswidrig handelnde (Gast-)Staat nicht (aus freien Stücken oder) auf einen Rechtsbehelf zu den eigenen Verwaltungsbehörden oder Gerichten hin Abhilfe geschaffen hat - eine Parallele zum auch in allgemeinen wirtschaftlich-gesellschaftlichen Motiven gründenden Erfordernis eines Rechtsschutzbedürfnisses, wie es der Inanspruchnahme von knappen gerichtlichen Ressourcen vorgeschaltet ist. Die Voraussetzung, zunächst „local remedies“ erfolglos auszuschöpfen, wird aber auch in zahlreichen BITs explizit genannt. ${ }^{210}$ In ihrer Reichweite ist sie allerdings teils diffus, weil damit noch nicht klar wird, ob und welche (zeitliche, sachliche, sonstige) Qualitätsanforderungen der innerstaatliche Rechtsschutz erfüllen muss, ob dieser (effektiv) nur vor staatlichen Gerichten und durch diese gewährleistet werden kann und wie weit eine angerufene ISDS-Einrichtung den klägerischen Vortrag insoweit prüfen muss. Ähnlich verhält es sich bei einem „,denial of justice“:211 Sieht ein Gastland (etwa gegen Parlamentsgesetze) überhaupt keine (innerstaatliche) gerichtliche Kontrolle vor, so besteht nur noch bzw. sofort die Möglichkeit einer Prüfung durch internationale Gerichte oder Schiedsgerichte. Billigen jedoch Gerichte Maßnahmen ,ihres“ Staates, ohne sie einer (durch nationales [Verfassungs-]Recht geforderten) intensiven Kontrolle zu unterziehen oder begehen sie gravierende Verfahrensfehler oder stellen kaum nachvollziehbare rechtliche Erwägungen an, ohne dass eine übergeordnete Instanz dies korrigiert, wäre eine bereits vor abschließender Entscheidung auf nationaler Ebene eröffnete Anrufung einer (unparteiischen) internationalen Stelle auch zur Verhütung weiterer Beeinträchtigungen wichtig, wenn insoweit keine Ausnahme von der ,local remedies rule" gilt, unabhängig davon, ob im ISDS-Verfahren solches Verhalten dann zudem als „unfair“ oder „vollem Schutz“ nicht entsprechend gewertet würde. ${ }^{212}$ Umgekehrt ist es zumindest primär eine Frage nationalen Rechts, ob ein Staat durch diesbezügliche eigene Vorschriften (etwa in ,investment codes") Meinungsverschiedenheiten mit ausländischen Investoren unmittelbar oder doch unter gewissen Bedingungen der Befassung durch ISDSStellen öffnet; auf dieses grundlegende staatstheoretische und verfassungsrechtliche Problem ist später noch einzugehen. ${ }^{213}$ Hier soll zunächst nur vermerkt werden, dass angesichts vertikaler und horizontaler Verknüpfung von ISDS-Mechanismen (zunächst national/inter-

208 Vgl. bereits Tsatsos, Die Rechtsprechung der ICSID-Schiedsgerichte: Zwischen Homogenität und Heterogenität, Diss. HU Berlin 2008, 44 ff.; Lehavi/Licht, Yale Journal of International Law 36 (2011), 115 (161 f.).

209 Vgl. Gessel-Kalinowska vel Kalisz/Czech, in: Legum (Fn. 88), 200 ff.; oben, B.II.4.

210 Etwa bei Option für „,arbitrage international“ in Art. 12 Abs. 2 BIT Belgien - Togo 2009, https:// investmentpolicy.unctad.org/international-investment-agreements/treaty-files/420/download.

211 Vgl. Winkler, Denial of justice im internationalen Investitionsschutzrecht: Grundlagen und aktuelle Entwicklungen, 2015, 16 ff.; ferner unten, bei Fn. 373.

212 Zur Abgrenzung Winkler, aaO, 8 f., $23 \mathrm{ff}$.

213 Unten, C.IV.2.b), D.I.3; hierzu auch Hahn und Hatje, in: Ludwigs/Remien (Fn. 57), 201 ff., 237 ff. 
national, hier global, regional und subregional; aber auch bei räumlichen und sachlichen Überlappungen auf der gleichen Ebene) eine Bereinigung des vorhandenen „Flickenteppichs“ dringlich erscheint, was allerdings auch, wie im Verhältnis ICSID-Streitbeilegung und EU-Gerichtsbarkeit schon akut geworden, ${ }^{214}$ Konsequenzen für das Verhältnis zwischen gerichtlichen, gerichtsähnlichen und außergerichtlichen Institutionen und Verfahren hat und insoweit ebenfalls Reformbedarf zeitigt. Das Beispiel von CETA $^{215}$ verdeutlicht überdies, dass, wenn Kompetenzen für Investitionsförderung und -schutz zwischen einer regionalen Wirtschaftsgemeinschaft und ihren Mitgliedsländern aufgeteilt sind, die dadurch entstandene Dreiecksbeziehung gegenüber dritten Heimatländern von Investoren die Komplexität noch erhöht, wenn und weil materielles Recht und Streitbeilegung auf der Seite der REC (hier der EU) je verschiedenen Ebenen zugewiesen sind, dies aber mit den völkervertraglich getroffenen Regeln nicht abgestimmt ist (und auch nicht ohne weiteres gelöst werden kann, solange einschlägige multilaterale Übereinkünfte die volle Mitgliedschaft internationaler Organisationen nicht vorsehen ${ }^{216}$ ).

\section{Einrichtungen und Verfahren mit globaler Reichweite und Relevanz für Afrika \\ 1. ICSID}

a) Organisation, Kompetenzen und Verfahren

1965 nach mehrjähriger Diskussion abgeschlossen, im Jahre darauf in Kraft getreten, beinhaltet das allen Staaten, nicht nur Weltbank-Mitgliedern (Art. 67) zum Beitritt offen stehende Übereinkommen über das International Centre for Settlement of Investment Disputes (ICSID) in Kap. I Bestimmungen sowohl über Errichtung (Art. 1), Sitz (Art. 2) und Organe (Art. 3), nämlich Administrative Council (Art. 4 ff.) und Sekretariat (Art. $9 \mathrm{ff}$.), als auch über Panels (von „,conciliators“ wie von „,arbitrators“, Art. 12 ff.), ferner über Finanzierung (Art. 17) und für die für eine intergouvernementale Organisation typischen Status-, Privilegien- und Immunitäten-Regelungen (Art. 18 ff.). Dem Zweck von ICSID, nämlich ,to provide facilities for conciliation and arbitration of investment disputes between Contracting States and nationals of other Contracting States" (diese definiert in Art. 25 Abs. 2) im Einklang mit den Vorgaben des Übereinkommens (Art. 1 Abs. 2) tragen weitere Kapitel eingehender Rechnung. Allgemein steckt Kap. II zunächst die ,jurisdiction“ des Zentrums ab, die regelmäßig exklusiv ist (Art. 26 S. 1), wobei aber eine Erschöpfung innerstaatlicher Rechtsbehelfe als Voraussetzung für einen „consent to arbitration“ vorbehalten werden darf

214 Slowakei vs. Achmea BV (EuGH, Rs. C-284/16, 6.3.2018); vgl. Wiedmann, in: Ludwigs/Remien (Fn. 57), 225 ff.; UNCTAD, IIA Issues Note 3, Dez. 2018, 2 ff.; unten, IV.1.b), am Ende.

215 Vgl. BVerfGE 143, 65 ff. (13.10.2016); dazu Proelß, ZEuS 19 (2016), 401 ff.; Hoffmann, ZEuS 19 (2016), 459 ff.; EuGH, 30.4.2019, Rs. 1/17, ECLI:EU:C:2019:341.

216 Wichtigste Ausnahme ist die WTO, s. Art. XI Abs. 1 des WTO-Abkommens, https://www.wto.or g/english/docs_e/legal_e/04-wto_e.htm. 
(Art. 26 S. 2). Mittel diplomatischen Schutzes ${ }^{217}$ dürfen erst bei Nichterfüllung eines „award“ eingesetzt werden (Art. 27). Kap. III widmet sich dem Verfahren der inhaltlich in Art. 34 genauer beschriebenen „conciliation“; thematisiert werden das Verlangen nach Schlichtung (Art. 28), die Errichtung einer „conciliation commission“ (Art. 29 ff.) sowie der Ablauf der ,proceedings“ (Art. $32 \mathrm{ff}$.), die mit einem Bericht des Schlichters enden (Art. 34). Vorbringen in diesem Verfahren darf vorbehaltlich anderer Vereinbarung weder vor einem Schieds- noch einem ordentlichen Gericht noch sonst verwendet werden (Art. 35). Kap. IV befasst sich mit „,arbitration“ aufgrund eines „request“ (Art. 36), der Bestellung eines „tribunal“ (Art. 37 ff.), bestehend aus einer oder einer ungeraden Zahl von Personen, der Normierung von dessen ,powers and functions“ (Art. $41 \mathrm{ff}$.), basierend auf einer Kompetenz-Kompetenz (Art. 41 Abs. 1), und schließlich dem Schiedsspruch (Art. $48 \mathrm{f}$.) sowie Regeln betreffend dessen „,interpretation “, „revision“ und ,annulment" (Art. 50 ff.); am Schluss werden „recognition“ (Art. 53) und „enforcement“ (Art. 54 f.) behandelt. Kap. V. - VII. enthalten allgemeine Vorschriften über „replacement“ und „disqualification“" von Schlichtern oder Schiedsrichtern (Art. 56 ff.), Verfahrenskosten (Art. 59 ff.) und Verfahrensort (Art. 62 f.). Vor Kapiteln zu Übereinkommensänderungen (IX., Art. 65 f.) und Schlussbestimmungen (X., Art. 67 ff.) normiert Kap. VIII (Art. 64) für Beilegungen von Meinungsverschiedenheiten zwischen Übereinkommensparteien nach Scheitern von Verhandlungen und mangels anderweitiger Vereinbarung eine Zuständigkeit des Internationalen Gerichtshofs (ICJ). ${ }^{218}$ Auf Grundlage von Art. 6 Abs. 1 des Übereinkommens hat der „administrative council“" zum einen allgemeine ,administrative and financial regulations“ erlassen (lit. a]), ${ }^{219}$ vor allem aber (gemäß lit.b]) für Schlichtungs- und Schiedsverfahren relevante ,institution rules“220 (zum „request") und daran anknüpfende „,conciliation rules“ und „arbitration rules“. ${ }^{221}$ Ebenfalls gestützt auf Art. 6 ist 1978 eine zweite Säule in Gestalt der ICSID Additional Facility ${ }^{222}$ errichtet worden, die den Anwendungsbereich der Konvention erweitert, zum einen auf Verfahren (betr. „conciliation“ wie „arbitration“), bei denen eine Seite keine Übereinkommenspartei oder deren ,national“ ist, sodann auf Konstellationen, bei denen die Streitigkeit nicht unmittelbar aus der Investition herrührt, und

217 Hierzu Resolution 62/67 der U.N.-Generalversammlung v, 6.12.2007, https://www.un.org/Depts/ german/gv-62/band1/ar62067.pdf.

218 https://www.icj-cij.org/en. Vgl. Art. 93 U.N.-Charta; Art. 34 ff. des ICJ Statute, https://www.icj-c ij.org/en/statute; dazu Tanaka (Fn. 191), 128 ff.

219 Stand 2006, https://icsid.worldbank.org/resources/rules-and-regulations/convention/administrativ e-and-financial-regulations.

220 Stand 2003, https://icsid.worldbank.org/resources/rules-and-regulations/convention/institution-rul es.

221 Stand 2006, https://icsid.worldbank.org/resources/rules-and-regulations/convention/conciliation-r ules, https://icsid.worldbank.org/resources/rules-and-regulations/convention/arbitration-rules; dazu bereits oben, Fn. 60; ferner unten, D.III.2. -4 .

$222 \mathrm{http} / /$ icsidfiles.worldbank.org/icsid/icsid/staticfiles/facility-archive/facility-en.htm; zu spezifischen ,rules“ oben, Fn. 73. 
schließlich auf ,fact-finding proceedings“ ${ }^{223}$ Dieser zusätzliche Mechanismus vollzieht sich ebenfalls nach (den regulären weithin nachempfundenen) administrativ-finanziellen, Schlichtungs- und Schiedsverfahrensregeln sowie speziellen Vorschriften zum Verfahren der Tatsachenermittlung (in vier Anhängen zu den Additional Facility Rules), jeweils unter Verwaltung des ICSID-Sekretariats (Art. 2 i.V.m. Art. 1 Abs. 3 dieser Rules). Eine erneute Reform der Durchführungsregeln ist im Gange. ${ }^{224}$ Neben und ergänzend zu ICSID wurde Ende der 1980er Jahre ebenfalls als Teil der Weltbankgruppe die Multilateral Investment Guarantee Agency ${ }^{225}$ geschaffen. Das 2010 leicht geänderte Übereinkommen ${ }^{226}$ unterstreicht schon in der Präambel, dass ,the flow of foreign investment to developing countries would be facilitated and further encouraged by alleviating concerns related to non-commercial risks. "Zur Erfüllung dieser in Art. 2 Abs. 1 (allgemeiner) formulierten Hauptaufgabe soll die ebenfalls rechtsfähige Weltbank-"Schwester"-Einrichtung (Art. 1 Abs. 2) nach Art. 2 Abs. 2 einerseits ,issue guarantees" gegen ,non-commercial risks" (beschrieben in Art. 11) in Bezug auf bestimmte ,eligible“ Investitionen (Art. 12) in einem Mitgliedstaat (Art. 61), die aus anderen Mitgliedsländern fließen (s. Art. $13 \mathrm{ff}$.), andererseits „carry out appropriate complementary activities to promote the flow of investments to and among development member countries" (klassifiziert in Anhang A, Art. 3 lit.c] des Übereinkommens); präzisiert wird dies in Art. 23. Art. 19 sieht eine Zusammenarbeit mit und Abrundung von einschlägigen Aktivitäten nationaler Investitionsgarantie-Stellen ${ }^{227}$ vor, die auch nach Art. 21 mit privaten Erst- und Rückversicherern erfolgen soll; Art. 18 normiert bei Leistungen den Eintritt in die Rechte des entschädigten Investors (subrogation ${ }^{228}$ ). Bestimmungen über Streitbeilegung betreffen sowohl das Verhältnis von MIGA und Mitgliedstaaten (auch im Fall der Rechtsnachfolge, Art. 57 Abs. 2) als auch in Bezug auf ,holders of a guarantee or insurance"; hierfür sieht Art. 58 ein Schiedsverfahren vor, während in der zuerst genannten Beziehung Anhang II zum MIGA-Übereinkommen auf ein Vorgehen im Wege von „,negotiation“ (Art. 2), „conciliation“ (Art. 3) und „arbitration“ (Art. 4) verweist, wobei Schlichtung entweder statt oder vor einem Schiedsverfahren erfolgen kann. Der Maßstab für die rechtliche Beurteilung durch Schiedsrichter deckt sich weithin mit dem des ICSID-Übereinkommens, ist jedoch teils breiter gefasst, enthält aber keine Abstufung zwischen von den Parteien Vereinbartem und weiteren heranzuziehenden Regelungen. Denn Art. 4 lit. g) des Anhangs zu Art. 57 der MIGA-Konvention besagt: „The Tribunal shall, in any dispute within the scope of this Annex, apply the provisions of this Convention, any relevant agreement between the parties to the dispute, the Agency's by-laws and regulations,

223 Näher dazu unten, D.III.1.

$224 \mathrm{https} / /$ icsid.worldbank.org/resources/rules-and-regulations/icsid-rules-and-regulations-amendme nt-working-papers; dazu https://africaarbitration.org/2018/10/04/commentary-on-icsids-new-ruleproposals-by-cherie-blair-cbe-qc-and-sophia-louw/.

2251988 Inkrafttreten des Gründungsabkommens (Fn. 148), https://www.miga.org/miga-convention.

226 Durch Entschließung Nr. 86 des Gouverneursrats v. 30.7.2010 (BGB1. 2011 II, 1261).

227 Vgl. bereits oben, B.III.2.

228 Dazu auch oben, bei Fn. 38 und 53. 
the applicable rules of international law, the domestic law of the member concerned as well as the applicable provisions of the investment contract, if any" (S. 1), während es in Art. 42 Abs. 1 des ICSID-Übereinkommens heißt: „The Tribunal shall decide a dispute in accordance with such rules of law as may be agreed between the parties. In the absence of such agreement, the Tribunal shall apply the law of the Contracting State party to the dispute (including its rules of the conflict of laws) and such rules of international law as may be applicable." Bei weiteren Punkten herrscht hingegen inhaltliche Übereinstimmung: „Without prejudice to the provisions of this Convention, the Tribunal may decide a dispute ex aequo et bono if the Agency and the member concerned so agree" (Art. 4 lit.g] S.2, entsprechend Art. 42 Abs. 3); „The Tribunal may not bring a finding of non liquet on the ground of silence or obscurity of the law" (Art. 4 lit. g] S. 3, analog Art. 42 Abs. 2). Weitere inhaltsgleiche Bestimmungen gelten für Anerkennung und Vollstreckung von Schiedssprüchen (Art. 4 lit. j] bzw. Art. 53 - 55). ${ }^{229}$

\section{b) ICSID und Afrika}

Von den 155 ICSID-Vertragsparteien (Stand 2020) kommt fast ein Drittel aus Afrika. Davon sind drei Länder (Äthiopien, Namibia und Guinea-Bissau) nur Signatarstaaten, und (bisher) nicht nach Art. 67 ff. am Übereinkommen teil nehmen lediglich Angola, Libyen und Südafrika. ${ }^{230}$ Bei den allerersten Schiedsverfahren waren (auf Beklagtenseite) afrikanische Staaten beteiligt (Marokko, Elfenbeinküste, Gabun, Kamerun etc.), ${ }^{231}$ wobei deren Zustimmung in der Regel noch auf einem Vertrag mit dem Investor und nicht auf einem IIA beruhte. Bis heute sind Länder aus Afrika in großem Umfang Partei von ICSID-Verfahren (im Herbst 2020 neu etwa Ägypten, Nigeria, Südsudan, Tansania) ${ }^{232}$, auch auf Basis der Additional Facility (Foresti v. Südafrika) ${ }^{233}$, ferner zuweilen im Rahmen von Schlichtungen

229 Näher dazu unten, E.II.

230 Aktuelle Liste: https://icsid.worldbank.org/sites/default/files/ICSID-3.pdf.

231 Holiday Inns S.A. and others v. Morocco (ICSID Case No. ARB/72/1); Adriano Gardella S.p.A. v. Côte d'Ivoire (ICSID Case No. ARB/74/1); Gabon v. Société Serete S.A. (ICSID Case No. ARB/76/1); Klöckner Industrie-Anlagen $\mathrm{GmbH}$ and others v. United Republic of Cameroon and Société Camerounaise des Engrais (ICSID Case No. ARB/81/2); ferner etwa AGIP S.p.A. v. People's Republic of the Congo (ICSID Case No. ARB/77/1); Le Cannu, ICSID Review - Foreign Investment Law Journal 33 (2018), $456 \mathrm{ff}$.

232 Gesenu S.p.A. v. Arab Republic of Egypt (ICSID Case No. ARB/20/45); Eni International B.V., Eni Oil Holdings B.V. and Nigerian Agip Exploration Limited v. Federal Republic of Nigeria (ICSID Case No. ARB/20/41); Qatar National Bank (Q.P.S.C.) v. Republic of South Sudan and Bank of South Sudan (ICSID Case No. ARB/20/40): Nachingwea U.K. Limited (UK), Ntaka Nickel Holdings Limited (UK) and Nachingwea Nickel Limited (Tanzania) v. United Republic of Tanzania (ICSID Case No. ARB/20/38).

233 ICSID Case No. ARB(AF)/07/1; bezogen u.a. auf den BIT mit der BLEU 1998, https://investmen tpolicy.unctad.org/international-investment-agreements/treaty-files/414/download. 
(zuletzt 2018 betreffend Gabun) ${ }^{234}$. Da zugleich Zahl und Teilnehmerkreis von BITs und anderen Abkommen mit Investitionsschutzregeln immer noch zunehmen, bewirkt auch diese Entwicklung eine hohe Inanspruchnahme der ICSID-Mechanismen. Zudem bietet das Washingtoner Zentrum auch (unterschiedlich intensive administrative) Dienstleistungen im Hinblick auf Schiedsverfahren nach UNCITRAL- und anderen Nicht-ICSID-Vorschriften an. ${ }^{235}$ Insofern kann hier durchaus von einem globalen Erfolgsmodell gesprochen werden, das jedoch nicht unumstritten ist. Die schiere Zahl von mehr als 1.050 bislang entschiedenen oder sonst erledigten Fällen (cases) ${ }^{236}$ besagt noch nicht zwingend etwas über die Qualität der konkreten Lösung und eine dauerhafte Befriedung von Konflikten, solange nicht für alle (potenziellen) Parteien von ,international investment disputes“ gleichwertige Alternativen verfügbar sind. Kritisch betrachtet wird insbesondere die in der Praxis unzulängliche Diversität von Schiedspersonen und -orten. ${ }^{237}$

\section{UNCITRAL}

Im Unterschied zu ICSID als Organisation mit eigener Völkerrechtspersönlichkeit erarbeitet die UN Commission on International Trade $\mathrm{Law}^{238}$ primär Regelungsentwürfe, entweder als Modell (und damit Harmonisierungsinstrument) für staatliche Gesetzgebung oder als „draft" eines multilateralen völkerrechtlichen Abkommens. Eine weitere Option ist die Einbeziehung von UNCITRAL Rules durch je individuelle Vereinbarung der Parteien an einer Meinungsverschiedenheit. Die jeweiligen Texte werden regelmäßig von Anwendungsempfehlungen begleitet. Insoweit beansprucht das Model Law for International Commercial Arbitration von 1985/2006 239 Anwendung auf „,kommerzielle“ Beziehungen jeder Art, ob vertraglich oder nicht (Fußnote $2 \mathrm{zu}$ Art. 1 Abs. 1), wobei allerdings Investitionsstreitigkeiten nicht explizit genannt sind. Dieser weite Anwendungsbereich ähnelt dem der New Yorker Konvention 1958 (in Art. II Abs. 1), während die Mauritius Convention on Transparency $(2014)^{240}$ sich auf „treaty-based investor-State arbitration“ bezieht, ebenso wie die „rules on transparency“ vom selben Jahr. ${ }^{241}$ Daneben existieren mehrere Dokumente zu (weit ge-

234 Société d‘Energie et d'eau du Gabon v. Gabonese Republic, ICSID Case No. CONC/18/1. Überblick in jährlichen ICSID Caseload Statistics (Special Focus - Africa), sowie der halbjährlichen Gesamtstatistik, zuletzt Issue 2020-2, 12.

235 Näher dazu unten, D.III.1.

2361023 Ende 2019, https://investmentpolicy.unctad.org/investment-dispute-settlement; dazu UNCTAD, IIA Issues Note - International Investment Agreements, Juli 2020, 1 ff.; 1061, davon 707 abgeschlossen zum 21.7.2020.

237 Dazu auch unten, G.I.

238 https://uncitral.un.org/; vgl. bereits Gramlich, RiA 2020, 3 (11 ff.).

239 https://uncitral.un.org/en/texts/arbitration/modellaw/commercial_arbitration.

240 https://uncitral.un.org/en/texts/arbitration/conventions/transparency.

$241 \mathrm{https} / /$ uncitral.un.org/en/texts/arbitration/contractualtexts/transparency. 
fasster, Schlichtung einbeziehender) Mediation, ${ }^{242}$ ältere „,conciliation rules“ $(1980)^{243}$ und ein 2018 reformiertes Modellgesetz „on international commercial mediation and international settlement agreements resulting from mediation" ${ }^{244}$ sowie die Singapore Convention on Mediation ebenfalls von 2018; ${ }^{245}$ auch hier werden ,commercial disputes“ in einem weiten Sinn erfasst. Sowohl das Mauritius- als auch das Singapurabkommen lassen als Vertragspartei auch ,regional economic integration organizations“ zu (Art. 8 bzw. Art. 12), ermöglichen andererseits einen Beitritt unter Vorbehalt und damit flexible Lösungen.Auf UNCITRAL-Vorschriften, insbesondere die Arbitration Rules, wird auch in IIAs oft Bezug genommen, als eine von mehreren Verfahrensoptionen, ebenso in nationalen ,investment codes" ${ }^{246}$ Darüber hinaus sind diese gouvernementalen Muster aber auch geeignet als Grundlage privater, nicht-gouvernementaler internationaler Streitbeilegung zwischen nichtstaatlichen Beteiligten. Ihre Generierung im allgemeinen UN-Kontext und daher unter umfassender Mitwirkung aller Betroffenen macht sie gerade auch für Staaten generell akzeptabel. Im Unterschied zu ICSID fehlt es aber an einer Institutionalisierung auf Dauer; Mediation wie Arbitrations Rules beschränken sich auf Verfahrens- und Entscheidungsregelungen.

\section{Weitere global ausgerichtete Stellen}

Für Interessenten weltweit werden Streitbeilegungsdienstleistungen auch über eigentliche Schiedsverfahren hinaus von einigen weiteren, teils schon langjährig aktiven Einrichtungen offeriert, die überwiegend nicht auf intergouvernementaler Grundlage beruhen. Anders ist dies nur beim Permanent Court of Arbitration (PCA) mit Hauptsitz in Den Haag, ${ }^{247}$ der durch zwei multilaterale Abkommen von 1899 und $1907^{248}$ geschaffen wurde, zunächst für die friedliche Beilegung internationaler Streitigkeiten zwischen Staaten (Art. 15/20 ff. bzw. Art. 37/41 ff.). Diverse allgemeine und spezifische Arbitration Rules, gestützt auf eine weite Auslegung von Art. 47 Abs. 1 des Übereinkommens von 1907 und heute inhaltlich angelehnt an die UNCITRAL-Regeln von $2010,{ }^{249}$ beziehen jedoch seit vielen Jahrzehnten die

242 Dazu näher unten, D.III.2.

243 https:/uncitral.un.org/en/texts/mediation/contractualtexts/conciliation.

244 https:/uncitral.un.org/en/texts/mediation/modellaw/commercial_conciliation.

$245 \mathrm{https} / /$ uncitral.un.org/en/texts/mediation/conventions/international_settlement_agreements.

246 Etwa in ICC-Verfahren gegen Libyen, z.B.Cengiz İnşaat Sanayi ve Ticaret A.S. v. Libya, https://i nvestmentpolicy.unctad.org/investment-dispute-settlement/cases/776/cengiz-v-libya; im Rahmen der ICSID Additional Facility und eines BIT mit Österreich hingegen Strabag SE v. Libya (ICSID Case No. ARB(AF)/15/1).

247 https://pca-cpa.org/en/home/.

248 https://docs.pca-cpa.org/2016/01/1899-Convention-for-the-Pacific-Settlement-of-International-Di sputes.pdf, https://docs.pca-cpa.org/2016/01/1907-Convention-for-the-Pacific-Settlement-of-Inter national-Disputes.pdf.

249 So die Einführung zu den Arbitration Rules 2012, https://docs.pca-cpa.org/2015/11/PCA-Arbitrat ion-Rules-2012.pdf. 
„gemischte“ Konstellation mit ein, dass „a State, State-controlled entity, or intergovernmental organization has agreed with one or more States, State-controlled entities, intergovernmental organizations, or private parties that disputes between them in respect of a defined legal relationship, whether contractual, treaty-based, or otherwise" in einem Schiedsverfahren nach PCA-Regeln beigelegt werden sollen (Art. 1 Abs. 1, Fassung 2012). Verhandelt wurden hier in der Regel „contract-based arbitrations“ gegen Äthiopien, Djibouti, Mauritius und Kap Verde. ${ }^{250}$ Bei der International Chamber of Commerce mit Sitz in Paris $^{251}$ ist seit 1923 ein ICC Court of Arbitration eingerichtet, als unabhängige Schiedseinrichtung, ${ }^{252}$ deren Statut in Anhang I der ICC Arbitration Rules (2017) ${ }^{253}$ festgelegt ist (Art. 1 Abs. 1), die aber nicht selbst über Streitigkeiten entscheidet; ,, it administers the resolution of disputes by arbitral tribunals, in accordance with the Rules of Arbitration of the ICC... The Court is the only body authorized to administer arbitrations under the Rules, including the scrutiny and approval of awards rendered in accordance with the Rules. It draws up its own internal rules, which are set forth in Appendix II (the "Internal Rules")" (Art. 1 Abs. 2). Die Arbitration Rules befassen sich nach einleitenden Vorschriften mit dem Beginn des Verfahrens, einer Mehrzahl von Parteien oder (zugrunde liegenden) Verträgen sowie einer Konsolidierung, dem Schiedsgericht und dem Verfahrensablauf, dem Schiedsspruch, den Kosten (zusammen mit Anhang III) und schließlich Verschiedenem. Weitere drei Anhänge (außer den bereits erwähnten) behandeln „case management techniques“, „emergency arbitrator rules“ und (neu) „expedited procedure rules“. Empfohlen werden auch eine Standard ICC Arbitration Clause für den Normalfall sowie zwei Modifikationen. Zum anwendbaren Recht besagt Art. 21: „The parties shall be free to agree upon the rules of law to be applied by the arbitral tribunal to the merits of the dispute. In the absence of any such agreement, the arbitral tribunal shall apply the rules of law which it determines to be appropriate“; das Schiedsgericht soll dabei „take account of the provisions of the contract, if any, between the parties and of any relevant trade usages", jedoch ,the powers of an amiable compositeur" nur ausüben oder „ex aequo et bono“ entscheiden, wenn es von den Parteien hierzu ermächtigt wurde. Sehr viel knappere Mediation Rules $(2014)^{254}$ sehen die Bestellung eines neutralen Dritten, des Mediators, vor, der die Parteien bei der Beilegung ihres Streits unterstützt (Art. 1 Abs. 2), wenn sich diese nicht rechtzeitig auf eine an-

250 https://pca-cpa.org/en/cases/; ferner (im Rahmen des OIC Investment Agreement 1981, unten, Fn. 286) Kontinental Conseil Ingénierie v. Gabonese Republic, PCA Case No. 2015-25, https://w ww.italaw.com/cases/7560.

251 https://iccwbo.org/.

252 Oben, Fn. 74. Zu afrikanischen Fällen etwa Pan African Minerals v. Burkina Faso, http://www.mi ning.com/pan-african-minerals-2-2b-claim-giant-manganese-deposit-contract-dismissed-court/; Dig Oil v. Demokratische Republik Kongo, https://www.businesslive.co.za/bd/companies/energy /2019-03-07-drc-ordered-to-pay-sa-oil-company-617m-for-failing-to-honour-contracts/.

253 https://iccwbo.org/dispute-resolution-services/arbitration/rules-of-arbitration/. 2021 wird eine neue Fassung in Kraft treten, https://iccwbo.org/dispute-resolution-services/arbitration/rules-of-ar bitration/rules-of-arbitration-2021/.

254 https://iccwbo.org/dispute-resolution-services/mediation/mediation-rules/. 
dere Art von „settlement procedure“ einigen (Art. 1 Abs. 3). Für die Durchführung besagt Art. 7: „In establishing and conducting the mediation, the Mediator shall be guided by the wishes of the parties and shall treat them with fairness and impartiality. Each party shall act in good faith throughout the mediation" (Abs. 3, 4). Hierfür werden ebenfalls diverse Musterklauseln angeboten. Investitionsstreitigkeiten betrafen hier etwa Burkina Faso, Senegal und Äquatorial-Guinea. ${ }^{255}$ Das Arbitration Institute der Stockholmer Handelskammer (SCC) als Teil dieser privaten Organisation schwedischen Rechts ${ }^{256}$ bezeichnet sich selbst als zweitgrößte Einrichtung für die Beilegung von Investitionsstreitigkeiten, die in ca. 120 BITs als zuständiges Forum vorgesehen sei. Maßgeblich hierbei sind eigene Arbitration und auch Mediation Rules (von 2007 bzw. 2014), ${ }^{257}$ die teils mit den UNCITRAL-Regeln verknüpft sind. Bedeutung erlangte dies etwa gegenüber Benin. ${ }^{258}$ Der seit 1981 unter diesem Namen agierende London Court of International Arbitration (LCIA) ${ }^{259}$ hat eine dreiteilige Struktur, zu der neben dem LCIA Arbitration Court noch eine gemeinnützige Gesellschaft (company) und ein Sekretariat gehören. Der LCIA Court bietet ohne weitere Voraussetzungen sowohl ,arbitration“ als auch „mediation“ und andere „ADR services“ (etwa ,expert determination" oder ,,adjudication") an und verfügt für die ersten beiden Bereiche über aktuelle ,rules“. Auch besteht eine Kooperation mit dem Dubai International Financial Cent$\mathrm{re}^{260}$ in Form eines gemeinsam betriebenen Arbitration Centre (DIFC - LCIA) ${ }^{261}$ seit 2008, während eine ähnliche Zusammenarbeit mit Mauritius (LCIA - MIAC) 2018 beendet wurde, ${ }^{262}$ im Hinblick auf Indien verwendet der LCIA Court seit 2016 spezielle Schieds- und Mediationsregeln. ${ }^{263}$

255 Sanderson, 13.3.2019, https://globalarbitrationreview.com/icc-panel-dismisses-claim-against-bur kina-faso; Ballantyne, 14.2.2020, https://globalarbitrationreview.com/icc-panel-decides-australia n-battle-over-senegal-oilfield; Fitzpatrick v. Equatorial Guinea, 23.11.2011, https://jusmundi.com /en/document/decision/en-fitzpatrick-equatorial-guinea-limited-v-the-republic-of-equatorial-guin ea-award-friday-23rd-december-2011\#decision_6823.

256 Oben, Fn. 75.

257 https://sccinstitute.com/media/293614/arbitration_rules_eng_17_web.pdf; https://sccinstitute.com /media/49819/medlingsregler_eng_web.pdf.

258 https://investmentpolicy.unctad.org/investment-dispute-settlement/cases/820/puma-energy-v-beni n.

259 https://www.lcia.org/.

260 https://www.difc.ae/.

$261 \mathrm{http}: / /$ www.difc-lcia.org/.

$262 \mathrm{http}: / / \mathrm{miac} . \mathrm{mu} /$.

$263 \mathrm{http}: / /$ www.lcia-india.org/. 


\section{Einrichtungen und Verfahren mit regional/räumlich begrenzter Reichweite}

\section{Genereller Bedarf}

Bereits die Aktivitäten des LCIA zeigen, dass langjährige tätige Streitbeilegungseinrichtungen in europäischen Staaten ihren Fokus stärker auf Partnerschaften mit in anderen Kontinenten verankerten Partnerinstitutionen legen, nicht nur, weil zumindest eine Partei eines Konflikts dort ihren Sitz oder geschäftlichen Schwerpunkt hat, sondern auch wegen der für ein belastbares Ergebnis wichtigen Einbindung in jeweilige lokale Rechtskultur und gesamtgesellschaftlichen Kontext. Zudem sind insoweit personelle und finanzielle Überlegungen überaus bedeutsam, deren Berücksichtigung ebenfalls dazu beitragen dürfte, dass eine nachhaltige und faire Lösung aus Sicht der Parteien wie dritter Betroffener gefunden werden kann. Im Folgenden soll diese Regionalisierungstendenz, die sich bislang vornehmlich im nicht-gouvernementalen Bereich zeigt, wieder speziell für Afrika etwas detaillierter beleuchtet werden.

\section{Fokus auf Afrika}

Nur auf dem afrikanischen Kontinent und vorerst noch ohne einen institutionellen Überbau, dafür aber durch einige Mehrfach-Mitgliedschaften verkompliziert, widmen sich diverse völkerrechtliche Regelungen der Ausgestaltung (sub)regionaler Wirtschaftsgemeinschaften (RECs); einige davon haben supranationale Gerichtshöfe geschaffen, denen neben richterlichen Aufgaben auch weitere im Bereich außergerichtlicher Streitbeilegung, vornehmlich „arbitrage“/,arbitration“ übertragen sind. Dies ist zwar nicht der Fall beim Cour de Justice de 1'UEMOA, ${ }^{264}$ dessen Träger ausschließlich (acht) Staaten bilden, die auch der OHADA angehören, ${ }^{265}$ jedoch durchaus beim ECOWAS Court of Justice, der seine Basis sowohl in einigen (auch anderen) OHADA-Ländern als auch weiteren westafrikanischen Staaten hat. Nach dem maßgeblichen (geänderten) Protokoll (Art. 9 Abs. 5) ${ }^{266}$ übt dieser Gerichtshof bis auf weiteres auch die Kompetenzen des im revidierten Gründungsvertrag (Art. 16) ${ }^{267}$ vorgesehenen Arbitral Tribunal aus.Die Komoren und die Demokratische Republik Kongo sind hingegen Parteien des COMESA-Abkommens ${ }^{268}$ und der SADC, ${ }^{269}$ nur letztere auch der Economic Community of the Great Lakes Countries (ECGLC) ${ }^{270}$, während eine größere Zahl von OHADA-Mitgliedern auch (neben weiteren Staaten) in der Communauté Écono-

264 https://courdejusticeuemoa.org/.

265 Côte d'Ivoire, Senegal, Mali, Burkina Faso, Benin, Niger, Togo und Guinea-Bissau.

266 Zusatzprotokoll v. 19.1.2005, http://prod.courtecowas.org/wp-content/uploads/2018/11/Suppleme ntary_Protocol_ASP.10105_ENG.pdf; vgl. Naud et al. (Fn. 143).

267 V. 24.7.1993, https:/www.ecowas.int/wp-content/uploads/2015/01/Revised-treaty.pdf.

268 Common Market for Eastern and Southern African Countries, Gründungsvertrag 1993 (oben, Fn. 154).

269 V. 17.8.1992, https://www.sadc.int/files/8613/5292/8378/Declaration_Treaty_of_SADC.pdf.

270 http://www.cepgl.org/cepgl. 
mique des États d'Afrique Centrale (CEEAC) ${ }^{271}$ zusammengeschlossen ist und sechs zugleich an der Communauté Économique et Monétaire de l'Afrique Centrale (CEMAC) ${ }^{272}$ teilnehmen.Bei CEMAC hat ein Cour de Justice Communautaire eine dreifache Funktion: ,juridictionnelle, consultative et d'administration des arbitrages dans les matières relevant du droit communautaire de la CEMAC “ (Art. 22 des Errichtungsabkommens 2009273); in der letztgenannten handelt er aber nicht selbst als Entscheider, sondern „,nomme et confirme les arbitres, est informée du déroulement de l'instance et examine les projets des sentences conformément à son Règlement d'Arbitrage" (Art. 35 Abs. 3), dessen Normierung der Konferenz der Staatschefs obliegt (Art. 36 i.V.m. Art. 12 des revidierten Gründungsvertrags). Ein CEEAC-Gerichtshof, obschon bereits in Art. 16 des Errichtungsabkommens $1983^{274}$ vorgesehen, existiert bislang nicht, ebenso wenig sind ihm durch die Konferenz der Staatsund Regierungschefs nicht-richterliche Kompetenzen (nach Art. 16 Abs. 4 i.V.m. Art. 8 ff.) übertragen worden. Hingegen wurde 2005 auf der Grundlage des SADC-Vertrags ${ }^{275}$ ein Tribunal errichtet, welches nach Art. 16 Abs. 1 befugt ist ,to adjudicate upon such disputes as may be referred to it." Das gemäß Art. 16 Abs. 2 erlassene Protokoll beschränkt auch nach mehreren Reformen dessen Kompetenz allerdings auf richterliche und rechtsgutachterliche Angelegenheiten. ${ }^{276}$ Das „Protocol on finance and investment“" $(2006)^{277}$ sieht in Art. 24 nur Streitbeilegung zwischen Mitgliedstaaten vor, trifft in Anhang I jedoch Regeln speziell über „settlement of investment disputes“. Scheitert eine einvernehmliche Lösung und sind innerstaatliche Rechtsbehelfe ausgeschöpft, so eröffnet Art. 28 (Abs. 2) den Streitparteien Optionen, sich entweder auf die Einschaltung des SADC Tribunal oder ein ICSIDVerfahren (auch nach der Additional Facility) oder ein ad hoc-Verfahren nach UNCITRALRegeln zu einigen, wobei bei erfolglosem Verstreichen einer Frist die je aktuellen UNCITRAL Arbitration Rules gelten sollen (Abs. 3). Bis auf weiteres wird das SADC Tribunal freilich nicht tätig. ${ }^{278}$ Der revidierte COMESA-Vertrag sieht als Organe des Gemeinsamem Marktes u.a. einen Court of Justice mit zwei Instanzen vor (Art. 7 Abs. 1 lit. c]), dem sodann ein eigenes Kapitel (V.) gewidmet ist (Art. 19 - 36). Art. 28 weist dieser supranationalen Einrichtung auch ,jurisdiction under arbitration clauses“ (und „special agreements") $\mathrm{zu}$, wobei auf eine vertragliche Schiedsklausel abgestellt wird ,to which the Common Market or any of its institutions is a party“ (lit. a]). Die gemäß Art. 38 des Vertrags von diesem

271 https://ceeac-eccas.org/.

272 http://www.cemac.int/.

$273 \mathrm{http} / / /$ www.cemac.int/sites/default/files/inline-files/Convention_CJ.pdf, als Konkretisierung von Art. 48 des revidierten CEMAC-Vertrags (ebenfalls) v. 30.1.2009, http://www.cemac.int/sites/def ault/files/inline-files/traite_revise.pdf.

274 https://www.wipo.int/edocs/lexdocs/treaties/en/eccas/trt_eccas.pdf.

275 Oben, Fn. 269.

276 V. 7.8.2000, Art. 14 f., https://www.sadc.int/files/1413/5292/8369/Protocol_on_the_Tribunal_and _Rules_thereof2000.pdf.

277 V. 18.8.2006, https://www.sadc.int/documents-publications/show/1009.

$278 \mathrm{https} / / /$ www.sadc.int/about-sadc/sadc-institutions/tribun/. 
Gericht erlassenen, vom COMESA-Ministerrat (Art. 7 Abs 1 lit. b] i.V.m. Art. 9 - 12) gebilligten Arbitration Rules (2018), ${ }^{279}$ die vorbehaltlich zwingenden Rechts für alle Sachverhalte des Art. 28 lit. a) gelten (Art. 2 Abs. 2 lit. a]), befassen sich nach einer Einleitung mit Zusammensetzung und ,jurisdiction" des Schiedsgerichts, sodann mit Schiedsverfahren und -spruch sowie den Kosten; zwei Anhänge enthalten Regeln für ,emergency arbitration procedure" sowie (zwei) Formulare. Art. 31 sieht als ,anwendbares Recht vorrangig die Regeln an "designated by the Parties as applicable to the substance of the dispute" (Abs. 1), ansonsten ,the law determined by the conflict of law rules, which it considers applicable“ (Abs. 2). Das Schiedsgericht darf nur dann als ,amiable compositeur“ oder ,ex aequo et bono" handeln, wenn es hierzu von den Parteien ausdrücklich ermächtigt wurde und das anwendbare Schiedsverfahrensrecht dies zulässt (Abs. 3), und entscheidet in allen Fällen ,in accordance with the terms of the contract" und unter Beachtung der ,usages of the trade applicable to the transaction". Andererseits normiert Kap. XXVI des COMESA-Vertrags über ,investment promotion and protection“ nur diverse Verpflichtungen der Mitgliedstaaten, so dass aus dem Beitritt zu ICSID, MIGA und anderen ,multilateral investment agreements" (Art. 162) auch allein für sie Vorgaben zur Streitbeilegung folgen, und dies ebenfalls für die Umsetzung des Art. 159 gilt, also für (völkervertragliche wie innerstaatliche) Maßnahmen ,to encourage and facilitate private investment flows into the Common Market." Letztlich erweist sich damit die OHADA im Hinblick auf Schieds- und Mediationsverfahren wie auch auf die Rolle eines regionalen inter- bzw. supranationalen Gerichtshofs ${ }^{280}$ hierbei als ein spezieller Fall, dem am ehesten noch die Rechtslage in der East African Community $(\mathrm{EAC})^{281}$ ähnelt, wo der East African Court of Justice nach Art. 32 des Gründungsvertrags ebenfalls aufgrund von ,arbitrations clauses" tätig wird - die nach lit. c) auch in einem ,commercial contract or agreement“ enthalten sein können „,in which the parties have conferred jurisdiction on the Court" - und der Gerichtshof gemäß Art. 42 entsprechende Arbitration Rules erlassen hat. Die African Continental Free Trade Area (AfCFTA) als „Dach“, das zahlreiche RECs als Bausteine überdeckt (so beschrieben auch in der Präambel des Übereinkommens ${ }^{282}$ ), kennt vorerst eine Streitbeilegung nur zwischen Mitgliedstaaten (Art. 20 Abs. 1), deren Einzelheiten in einem besonderen Protokoll festgelegt sind (Abs. 2, 3). Ein „Protocol on investment" ist erst für die zweite Phase vorgesehen (Art. 7, 8, 23 Abs. 2); ob dort auch eine Investor-Staat-Streitbeilegung normiert wird und in welcher Weise, ist derzeit noch ungewiss.

279 https://comesacourt.org/wp-content/uploads/2019/11/COMESA-COURT-ARBITRATION-RUL ES-2018.pdf.

280 Dazu näher unten, D.IV.1.

281 Dazu schon Gramlich, RiA 2020, 3 (18 ff.); ferner Ssemmanda/Shikoli, African Journal of Commercial Law 1 (2019/2020), $173 \mathrm{ff}$.

282 V. 21.3.2018, https://au.int/sites/default/files/treaties/36437-treaty-consolidated_text_on_cfta_-_e n.pdf. 
3. Mechanismen mit größerer territorialer Reichweite, aber hoher Relevanz für Afrika

Zahlreiche afrikanische Staaten, davon 13 aus dem OHADA-Raum, ${ }^{283}$ sind Mitglieder der Organization for Islamic Cooperation (OIC) ${ }^{284}$ und insbesondere Parteien des Agreement on Promotion, Protection and Guarantee of Investments zwischen den Mitgliedsländern (1981). ${ }^{285}$ Nach Art. 16 (Abs. 1) des Übereinkommens muss jeder Gaststaat einem Investor (Art. 1 Nr. 6) das Recht auf Zugang zu seinem Gerichtssystem auch gegen solche eigenen Maßnahmen gewähren, die gegen die Investoren schützende Regeln verstoßen; alternativ ist ein Schlichtungs- bzw. Schiedsverfahren nach Art. 17 (i.V.m. Art. 16 Abs. 2) vorgesehen. Eine Kompetenz hierfür ist allerdings nicht dem Internationalen Islamischen Gerichtshof mit Sitz in Kuwait ${ }^{286}$ zugewiesen, vielmehr müssen einschlägige Verfahren vor anderen Einrichtungen durchgeführt werden, etwa dem PCA. ${ }^{287}$ Aus dem OHADA-Kreis gehören die Komoren, darüber hinaus aber auch diverse weitere (west- und nord)afrikanische Länder der Arabischen Liga ${ }^{288}$ an; auch in deren Rahmen besteht bezogen auf „Arab investors“ (Art. 1 Nr. 6, 8) ein Arab Investment Agreement (1980), ${ }^{289}$ das 2013 neu gefasst wurde. ${ }^{290}$ Bei Meinungsverschiedenheiten können sich die Streitparteien an gaststaatliche Gerichte oder an den Arab Investment Court wenden (Art. 21 - 23, 25). ${ }^{291}$ Sie können aber auch andere außergerichtliche Streitbeilegungsverfahren wählen, sowohl Mediation oder Schlichtung als auch „arbitration“ (Art. 24 i.V.m. Anhang). In diesem Fall haben sie die Option, ICSID-, ICC- oder jede andere Schiedsgerichtsbarkeit zu befassen, im Zweifel bilden die einschlägigen UNCITRAL-Regeln das anwendbare Recht. Hingegen spielte der Energy

283 Nicht Guinea, Zentralafrikanische Republik, Republik Kongo sowie Demokratische Republik Kongo.

$284 \mathrm{https} / / /$ www.oic-oci.org/states/?lan=en.

285 http://ww1.oic-oci.org/english/convenion/Agreement $\% 20$ for $\% 20$ Invest $\% 20 \mathrm{in} \% 20 \mathrm{OIC} \% 20 \% 20 \mathrm{E}$ n.pdf.

$286 \mathrm{http} / / / \mathrm{ww} 1$. oic-oci.org/english/convenion/1987/statute_of_the_international_islamic_court_ofju stice_en.pdf.

287 Etwa KCI v. Gabun, PCA Case No. 2015-25, 23.12.2016, https://jusmundi.com/en/document/dec ision/fr-kontinental-conseil-ingenierie-v-gabonese-republic-sentence-finale-friday-23rd-decembe r-2016\#decision_14; zur Ablehnung einer Annullierung unten, Fn. 449.

288 Vgl. Masters/Sergie, https://www.cfr.org/backgrounder/arab-league; zum Gründungsvertrag 1945 https://avalon.law.yale.edu/20th_century/arableag.asp.

289 26.11.1980, https://investmentpolicy.unctad.org/international-investment-agreements/treaty-files/ 2394/download.

$290 \mathrm{http} / / /$ arbitrationblog.practicallaw.com/investment-arbitration-in-the-middle-east-basic-trends-an d-developments-part-2/; https://www.oecd.org/mena/competitiveness/oecd\%20study_amended $\%$ 20arab\%20league\%20investment\%20agreement\%20(english).pdf; Ziadé, Arbitration 83 (2017), 48 ff.; Hasaan, ICSID Review - Foreign Investment Journal 34 (2019), 107 ff.; zur politischen Dimension von Investititionsschutzrecht Fink, ZaöRV 72 (2012), 483 ff.

291 Tanmiah v. Tunisia, Arab Investment Court, 2004, https://www.italaw.com/cases/1072; dazu Ben Hamida, Journal of World Investment \& Trade 7 (2006), 699 ff.; ferner https://www.iflr.com/artic le/b11sps7r4ktcdm/egypt-the-arab-investment-court; Gaffney, Columbia FDI perspectives, No. 181, Aug. 2016, $1 \mathrm{f}$. 
Charter Treaty (ECT), ${ }^{292}$ dem formal weltweit Staaten und RECs beitreten können (Art. 39, 41), für Afrika bislang in der Praxis keine Rolle, und damit auch nicht dessen Art. 26 über „settlement of disputes between an investor" (Art. 1 Abs. 7) und ,a Contracting Party“. Ob sich dies ändern wird, wenn eine vielfach vorgeschlagene Reform zustande kommt ${ }^{293}$ und zudem die aktuelle Streitbeilegung an neuere Konzepte angepasst wird, ist noch nicht absehbar.

\section{Vielfalt und Vielzahl von (Schieds-)Einrichtungen in und für Afrika}

Eine aktuelle nicht-amtliche Liste nennt insoweit fast 80 Institutionen. ${ }^{294}$ Diese werden in der Regel von privaten bzw. nicht-gouvernementalen Stellen oder Vereinigungen getragen, auch wenn ihre Gründung auf ein (besonderes) Gesetz zurückgeht, wie etwa in Ruanda der Kigali International Arbitration Centre (KIAC) ${ }^{295}$ oder in Kenia der Nairobi Centre for International Arbitration (NCIA). ${ }^{296} 40$ Jahre bereits besteht das Cairo Regional Centre for International Commercial Arbitration (CRCICA), ${ }^{297}$ gestützt auf ein Abkommen zwischen der ägyptischen Regierung und dem Asian African Legal Consultative Committee, ${ }^{298}$ und 1990 wurde in dessen Rahmen das Institute of Arbitration and Investment errichtet, dem u.a. 2001 als weiterer Teil ein Mediation and ADR Centre folgte. ${ }^{299}$ Bedeutsam ist auch die Arbitration Foundation of Southern Africa (AFSA), ${ }^{300}$ die Streitigkeiten verschiedenen ,divisions“" zuordnet, nämlich ,domestic“, ,international" und SADC, aber auch eine eigene „mediation division“ eingerichtet hat. Die Erweiterung auf grenzüberschreitende „,disputes“ erfolgt nach Erlass des südafrikanischen International Arbitration Act 2017. ${ }^{301}$ Jedoch wird bereits seit 2016 in Marokko ein Casablanca International Mediation and Arbitration Centre (CIMAC) ${ }^{302}$ tätig, als unabhängige private Institution ebenfalls mit dem Anspruch, ein ,unique dispute resolution centre“ für den ganzen Kontinent zu werden. Dem

292 Oben, Fn. 15; Nacimiento, in: Legum (Fn. 88), 349 ff.

293 Vgl. Nyamongo/Naymongo, Energy Infrastructure Investments in East Africa and the Relevance of the Energy Charter Treaty, 2019, 32 ff., https://www.energycharter.org/fileadmin/DocumentsM edia/Occasional/Energy_Infrastracture_Investment_in_East_Africa.pdf; ferner https://www.iisd.o $\mathrm{rg} /$ projects/energy-charter-treaty.

294 https://africaarbitration.org/resources/africa-arbitration-institutions/.

295 https://kiac.org.rw/new/.

296 https://ncia.or.ke/; ferner https://afaa.ngo/page-18097/9170468; Muigua, Promoting International Commercial Arbitration in Africa, 2013, $13 \mathrm{f}$.

297 https://crcica.org/; dazu auch https://www.iarbafrica.com/en/news-list/17-news/1110-crcica-recen t-caseload-1 st-quarter-2019.

298 https://crcica.org/History.aspx; http://www.aalco.int/about (Ziff. K.).

299 http://www.aalco.int/arbitrationCRCICA; z.

300 https://arbitration.co.za/.

301 Act No. 15 of 2017, https://www.gov.za/sites/default/files/gcis_document/201712/41347internati onalarbitrationact15of2017.pdf.

$302 \mathrm{http}: / / \mathrm{cimac} \cdot \mathrm{ma} /$. 
dienen sollen Rules of Arbitration (2018) 303 $^{303}$ it breitem sachlichen und persönlichen Anwendungsbereich und die Einrichtung eines Court of Arbitration innerhalb des CIMAC mit der Aufgabe „to ensure the proper application“ der Regeln, wofür ihm „all the necessary powers to do so" eingeräumt sind (Rule $1.1 \mathrm{der}$ für diesen geltenden ,internal rules “). ${ }^{304} \mathrm{In}$ mehreren Staaten gibt es drei, in Nigeria und Südafrika sogar sechs Schiedszentren. ${ }^{305}$ Etwas unscharf kennzeichnet sich der 2019 errichtete Cour Africaine de Médiation et d'arbitrage (CAMAR) $)^{306}$ selbst als gemeinützige Organisation „,de droit international “, obwohl auch diese Stelle dem Recht des Sitzlandes (Marokko) unterliegt; die in elf Kammern gegliederte Einrichtung will ermöglichen, afrikaweit afrikanische Fälle auf afrikanischem Boden durch international anerkannte afrikanische Schiedsrichter beilegen zu lassen. Zudem gibt es Kooperationen sowohl im MENA-Raum (Nordafrika und Mittlerer Osten, etwa das Dubai International Arbitration Centre ${ }^{307}$ als deren „Herz“ sowie die gemeinschaftliche Einrichtung von LCIA und Dubai International Financial Centre, das DIFC-LCIA ${ }^{308}$ ) als auch mit asiatischen Stellen; AFSA etwa hat mit einem Schiedszentrum aus Shanghai das China-Africa Joint Arbitration Centre (CAJAC) in Johannesburg installiert. ${ }^{309}$ In etwas anderer Richtung besteht eine vertraglich besiegelte Zusammenarbeit zwischen OHADA (CCJA) und ICC (Court of Arbitration). ${ }^{310}$ In Mauritius, wo eine der beiden Schiedseinrichtungen, der Arbitration and Mediation Center der dortigen Industrie- und Handelskammer $(\mathrm{MARC})^{311}$ vielfältige weitere Dienste offeriert, sind ebenfalls Kontakte mit Hongkong/ China vorhanden, ${ }^{312}$ während das Mauritius International Arbitration Centre (MIAC) einerseits eine vorübergehende Verbindung mit dem LCIA beendet hat, ${ }^{313}$ seit 2018 jedoch im

303 http://cimac.ma/wp-content/uploads/2019/03/CIMAC_Reglement_GB_04052018.pdf.

304 Vgl. El Mernissi/Salem, https://www.dlapiper.com/en/morocco/insights/publications/2017/12/le-r eglement-darbitrage-de-centre/.

305 http://arbitrationblog.kluwerarbitration.com/2019/10/01/arbitration-centres-in-africa-too-many-co oks/.

$306 \mathrm{https} / / /$ northafricapost.com/29792-african-mediation-arbitration-court-opens-in-morocco.html; http://arbitrationblog.kluwerarbitration.com/2019/06/04/african-dynamism-morocco-as-a-potenti al-hub-for-international-arbitration/.

$307 \mathrm{http} / / / \mathrm{www}$. diac.ae/idias/.

308 Vgl. schon oben, Fn. 261.

309 https://cajacjhb.com/. Weitere Sitze befinden sich in Shanghai, Peking und Shenzhen sowie in Nairobi und im OHADA-Raum, http://www.keatingchambers.com/wp-content/uploads/2017/12/ DW-CAJAC.pdf, https://cajacjhb.com/wp-content/uploads/2020/10/CAJAC_Rules_2020.pdf.

310 https:/iccwbo.org/media-wall/news-speeches/icc-ohada-partner-to-boost-arbitration-practices-inafrica/.

311 https://www.marc.mu/en.

312 https://www.marc.mu/en/about-us/our-partners.

313 Oben, Fn. 262; dazu auch https://africaarbitration.org/2018/07/20/lcia-exits-mauritius-and-a-newmiac-emerges-by-duncan-bagshaw. 
Rahmen des lokalen Gesetzes über internationale Schiedsgerichtsbarkeit ${ }^{314}$ mit dem PCA als Partner operiert. ${ }^{315}$

\section{Gemeinsamkeiten und Verknüpfungen}

Bei schieds- und anderer außergerichtlicher Streitbeilegung haben Kläger oder beide Streitparteien gemeinsam die Wahl, vor welcher Einrichtung, wo und nach welchen Verfahrensregeln sie eine Lösung anstreben. Meist wird auch explizit mit der Entscheidung für eine bestimmte Stelle diese ausschließlich zur Entscheidung berufen, ein zweites oder weiteres Verfahren über denselben Streitgegenstand würde daher als unzulässig behandelt. ${ }^{316}$ Probleme treten hingegen bei der Konkretisierung des je anwendbaren Rechts (insbesondere für die Entscheidung in der Sache) auf, wenn ein mit der spezifischen (Investitions-)Angelegenheit befasstes Gremium eine Mehrzahl von Rechtsquellen zu beachten hat. Wird auf Völkerrecht Bezug genommen, geht es allerdings oft um Verträge, so dass sich deren persönlicher, räumlicher oder auch zeitlicher Anwendungsbereich nach jeweils spezifischen Regeln voneinander abgrenzen lässt. ${ }^{317}$ Nicht nur zweiseitige, sondern multilaterale Abkommen enthalten zuweilen auch sachbezogen unterscheidende Zuordnungen, wie etwa Art. 11 des BIT zwischen Deutschland und Guinea 2006, ${ }^{318}$ Art. 18, 19 des AfCFTA-Übereinkommens $^{319}$ oder Art. 3 PAIC. ${ }^{320}$ Allgemein können auch Schiedsrichter generelle methodische Ansätze heranziehen, z.B. nach der späteren und/oder spezielleren Vorschrift fragen, dispositive Bestimmungen an zwingenden (ius cogens) messen und auch ansonsten auf die in der Wiener Vertragsrechtskodifikation (WVRK) ${ }^{321}$ niedergelegten Regeln zurückgreifen. Ein besonderes Problem werfen Meistbegünstigungsklauseln auf, deren Reichweite auch bei ,investment disputes“ in ICSID-Verfahren bereits zu analysieren war, nämlich in der Sache Menzies v. Senegal. ${ }^{322}$

314 Aktuelle Fassung des International Arbitration Act 2008, http://miac.mu/wp-content/uploads/201 9/01/mauritian-international-arbitration-legislation-handbook-updated-2016.pdf.

315 https://miac.mu/news/miac-commences-operations/.

316 Vgl. etwa Söderlund/Burova, ICSID Review - Foreign Investment Journal 33 (2018), 525 ff.

317 Dazu unten, IV.1.a); Waibel und Markert, in: Hofmann/Tams (Fn. 113), 29 ff., 53 ff.

318 Oben, Fn. 42.

319 21.3.2018, https://www.tralac.org/documents/resources/cfta/1963-agreement-establishing-the-afc fta-consolidated-text-signed-21-march-2018/file.html; vgl. Gramlich, RiA 2020, 3 (14 f.).

320 Oben, Fn. 158; vgl. bereits Gramlich, RiA 2020, 3 (15 f.); ferner Art. 105 EPA EU - ECOWAS/ UEMOA.

321 Wiener Übereinkommen v. 23.5.1969 über das Recht der Verträge (WVRK), BGB1. 1985 II, 926.

322 ICSID Case No. ARB/15/21, v. 21.7./1.8.2016, Rn. 129 ff., dazu Peréz-Aznar, ICSID Review Foreign Investment Journal 34 (201), 190 ff.; Nikièma, 12.12.2016, https://www.iisd.org/itn/2016 /12/12/mfn-clause-wto-gats-importing-consent-arbitration-third-party-bit-menzies-middle-east-af rica-aviation-handling-services-international-senegal/, zur Relevanz des GATS, ferner Allen/ Soave, in: Huerta-Goldman et al. (eds.), WTO litigation, investment arbitration, and commercial 


\section{Investitionsschutz an der Schnittstelle von Völker(vertrags)recht und staatlichem (Verfassungs-) Recht}

1. Allgemein

a) Geltungsanspruch und Anerkennung internationaler Rechtsquellen

Das in der Praxis vorfindliche Nebeneinander von völkerrechtlichen und innerstaatlichen Vorschriften sowie von intergouvernementalen und privaten, nicht-gouvernementalen Einrichtungen auch und gerade bei der Investor-Staat-Streitbeilegung nötigt dazu, Art, Inhalt und Umfang der Beziehungen zwischen internationaler und nationaler normativer Ebene genauer zu bestimmen, denn letztlich ergibt sich auch aus Art. 27 und Art. 46 WVRK, dass hierbei Völker- und Verfassungsrecht ineinandergreifen und die Maxime ,pacta sunt servanda“ (Art. 26 WVRK) nur dann gilt, wenn Zustandekommen und Inhalt einer Vereinbarung im internationalen Bereich unbedenklich sind. Hinzu kommt die erstmals in Europa seit 1951 (mit der Errichtung der Europäischen Gemeinschaft für Kohle und Stahl ${ }^{323}$ ) entwickelte Struktur supranationaler Organisationen, die trotz fehlender Staatsqualität (mangels Souveränität und Kompetenz-Kompetenz) ähnlich wie in Bundesstaaten rechtsverbindliche Vorschriften nicht nur für die Mitgliedsländer, sondern auch unmittelbar für deren Bürger und andere dort ansässige Personen treffen können. ${ }^{324}$ Auch hier setzt eine solche Legislativfunktion aber deren Anerkennung (dem Grunde wie ihrem Ausmaß nach) durch die „Herren“ des Organisationsverfassungsvertrags voraus, die ihrerseits intern-verfassungsrechtlich ausreichend legitimiert sein muss; ${ }^{325}$ wie bei allgemeinem Völkerrecht muss daher auch hier dem Geltungsanspruch eine nationale Öffnung für ,fremdes“ (und dem eigenen gegenüber vorrangiges) Recht gegenüberstehen und entsprechen. Dieser Ansatz ist nicht auf die EG, heute EU beschränkt, wo er sich etwa im Zusammenspiel von unionsrechtlichem Anwendungsvorrang und Art. 23 Grundgesetz (GG) zeigt, ${ }^{326}$ sondern generell auf das Verhältnis aller, auch der afrikanischen RECs zu ihren Mitgliedstaaten übertragbar. $^{327}$

arbitration, 2013, 45 ff.; generell zur Multilateralisierung durch MFN-Klauseln Schill, Berkeley Journal of International Law 27 (2009), $497 \mathrm{ff}$.

323 V. 18.4.1951, https://eur-lex.europa.eu/legal-content/DE/TXT/PDF/?uri=CELEX:11951K/TXT\& from $=\mathrm{DE}$.

324 Art. 288 Abs. 1, 2, 4 AEUV.

325 Unten, 2.a).

326 BVerfGE 89, $155 \mathrm{ff}$;; zuletzt BVerfG, 13.2.2020, 2 BvR 739/17, Rn. $119 \mathrm{ff}$., ECLI:DE:BVerfG:2020:rs20200213.2bvr073917.

327 Unten, 2.a). 
b) Übereinstimmung mit nationalem Verfassungsrecht

Dass eine völkerrechtlich korrekte Vorgehensweise (Verhandlungen, Paraphieren eines endgültigen Textes durch zur Außenvertretung befugte Personen, schließlich Ratifizierung durch das Staatsoberhaupt; s. Art. 6 ff. WVRK) allein nicht ohne weiteres die Schaffung und das Handeln Internationaler Einrichtungen vollumfänglich legitimieren kann, ist heute dem Grunde nach kaum zweifelhaft. Denn auch auf internationaler Ebene gilt für Verfassungsstaaten prinzipiell ein Zusammenwirken der (drei) verschiedenen Staatsfunktionen und wird damit auch, ja, in erster Linie eine maßgebliche Mitwirkung des unmittelbar demokratisch legitimierten Parlaments ${ }^{328}$ - gegebenenfalls sogar eine Form direkter Demokratie wie ein Volksentscheid ${ }^{329}$ - oder auch weiterer Verfassungsorgane erforderlich. Durch ein Zustimmungs- bzw. Vertragsgesetz wird jedoch auch eine normhierarchische Festlegung getroffen, sofern nicht höherrangiges innerstaatliches Recht, also die jeweilige Verfassung, hierzu abweichende Vorgaben enthält. Überdies ist auch die „Dritte Gewalt“ involviert, sei es, indem - passender Weise zwischen Unterzeichnung und Ratifikation - eine Kontrolle (regelmäßig durch ein Oberstes oder ein Verfassungsgericht) auf VerfassungsmäBigkeit hin erfolgt, sei es, dass die Vereinbarkeit von Vertragsregelungen mit der aktuellen Konstitution gutachterlich geklärt wird. Im einen wie im anderen Fall darf die endgültige Billigung des Vertragsschlusses durch die betreffende Partei der anderen Seite gegenüber erst stattfinden, wenn festgestellte Divergenzen durch eine Nachjustierung der Verfassung bereinigt worden sind. In der Zwischenzeit kommt dann lediglich eine (gegebenenfalls partielle) vorläufige Anwendung des Abkommens in Betracht. ${ }^{330}$ Ungeachtet der allgemeinen Verpflichtung jeder Partei, völkervertraglich eingegangene Bindungen in innerstaatliches Recht umzusetzen oder deren direkte Anwendbarkeit nicht zu behindern, bleibt es Aufgabe nationalen (Verfassungs-)Rechts, den dafür generell geeigneten Rang in der nationalen Rechtsordnung zu fixieren, der sich auch nicht notwendig mit der Einordnung von Völkergewohnheitsrecht (oder von allgemeinen Rechtsgrundsätzen) decken muss. Die Spannweite reicht hier vom Gleichrang mit einfachen (parlamentarischen) Gesetzen (und in der Folge einer möglichen Aufhebung der Sache nach durch spätere Gesetzgebung) über eine Zwischenstellung oberhalb des Gesetzes, aber unterhalb der Verfassung bis hin zu einem Gleichrang mit dieser oder gar einer Einordnung darüber, an der Spitze einer (internen) Vorschriftenpyramide. Im EU-Raum verdeutlichen dies Art. 216 und 218 AEUV bezüglich in der Zuständigkeit (auch) der Union fallender Übereinkommen; ${ }^{331}$ Deutschland unter-

328 Insoweit instruktiv BT-Drs. 18/6201 v. 30.9.2015, 1 ff. (Antrag BÜNDNIS 90/DIE GRÜNEN: Dem CETA-Abkommen so nicht zustimmen); BVerfG (Fn. 326), Rn. 133, 140.

329 Wie etwa in der Schweiz, s. Art. 141a i.V.m. Art. 140 Bundesverfassung der Schweizerischen Eidgenossenschaft 1999, https://www.admin.ch/opc/de/classified-compilation/19995395/202001 010000/101.pdf.

330 Etwa bei CETA ab September 2017; dazu BT-Drs. 18/8391 v. 10.5.2016, 1 f., https://ec.europa.eu /trade/policy/in-focus/ceta/ceta-explained/index_de.htm.

331 Vgl. EuGH, 27.2.2018, Rs. C-266/16, Rn. 45 ff. 
scheidet hier im Rahmen eigener Kompetenzen zwischen Verträgen über Gegenstände der Bundesgesetzgebung (Art. 59 Abs. 2 S. 1 GG) - bei BITs folgt diese (als ausschließliche) aus Art. 73 Abs. 1 Nr. 1, 5 - und „allgemeinen Regeln des Völkerrechts“ (Art. 25), bei denen auch eine besondere Art der Normenkontrolle durch das Bundesverfassungsgericht (BVerfG) vorgesehen ist (Art. 100 Abs. 2 GG). ${ }^{332}$ Vor allem frankophone afrikanische Staaten (aus dem OHADA-Raum) lehnen sich insoweit an das Muster der französischen Verfassung von $1958^{333}$ an. So besagt etwa die Verfassung Gabuns ${ }^{334}$ von 1991, „commercial“ und ,international organization treaties“ bedürften gesetzlicher Billigung (Art. 114 Abs. 1), (nur) Veränderungen des Staatsgebiets betreffende Regeln allerdings eines Volksentscheids (Abs. 4); gemäß Art. 113 Abs. 1 obliegt die Aushandlung von Staatsverträgen und anderen Abkommen dem Präsidenten der Republik, eine Ratifizierung durch diesen setzt aber ein zustimmendes Votum in Gesetzesform und eine „verification of their constitutionality“ durch das Verfassungsgericht voraus. Fast identisch damit sind die Vorschriften in Art. 95, 96 Abs. 1 - 3 und 97 der senegalesischen Verfassung (2001) (35 $^{33}$ und in Art. 213, 214 und 216 der Konstitution der Demokratischen Republik Kongo (2011). ${ }^{336}$ Zum innerstaatlichen Rang besagen diese Verfassung in Art. 215 und diejenige Senegals in Art. 98, ordnungsgemäß ratifizierte oder gebilligte ,traités“ oder ,accords“ erlangten mit ihrer Publikation „une autorité supérieure à celle des lois, sous réserve, pour chaque accord ou traité, de son application par l'autre partie“. Innerhalb der Europäischen Gemeinschaft sind (in der Regel mit oder auch zwischen Staaten, die diesem Verbund erst nachher beigetreten sind) ebenfalls nicht selten „Intra“-BITs geschlossen worden; ${ }^{337}$ der Europäische Gerichtshof hat insoweit Vertragsverletzungsklagen der Kommission im Hinblick auf die auch Drittländern gegenüber verbürgte Kapitalverkehrsfreiheit (heute Art. 63 ff. AEUV) mehrfach stattgegeben, wegen Unvereinbarkeit mit dem vorrangigen Primärrecht;; 382020 wurde

332 Vgl. etwa BVerfG, 3.7.2019, 2 BvR 824/15, Rn. 16 ff., ECLI:DE:BVerfG:2019:rk20190703.2bvr082415.

333 Art. 52 - 55, https://www.conseil-constitutionnel.fr/sites/default/files/as/root/bank_mm/constituti on/constitution.pdf.

334 https://www.refworld.org/pdfid/3ae6b53510.pdf, Fassung 2011.

$335 \mathrm{https} / / /$ www.sec.gouv.sn/lois-et-reglements/constitution-du-s\%C3\%A9n\%C3\%A9gal, Fassung 2016.

336 http://aceproject.org/ero-en/regions/africa/CD/rdc-constitution-2011/view; ferner Art. 149 - 151 der Verfassung von Guinea 2010, https://www.constituteproject.org/constitution/Guinea_2010.pd f?lang=en.

337 Etwa zwischen den Niederlanden und Ungarn 1987; dazu Emmis International Holding B.V. et al. v. Ungarn, ICSID Case No. ARB/12/02, 16.4.2014; ferner Sackmann, SchiedsVR 2015, 15 (17).

338 EuGH, 3.3.2009, Rs. C-205/06 (ECLI:EU:C:2009:118) und Rs. C-249/06 (ECLI:EU:C:2009:119); dazu etwa Lehavi/Licht, Yale Journal of International Law 36 (2011), 115 (157 f.). 
daher ein Abkommen zur Beendigung solcher Verträge geschlossen. ${ }^{339}$ Seit deren Gestaltwandel zur Europäischen Union wären neue BITs der Unionsmitglieder auch im Verhältnis zu Drittstaaten kein Gegenstand nationaler Kompetenz mehr ${ }^{340}$. Allerdings hat sich gezeigt, dass sich dies auf das Verhältnis von EU-Gerichtsbarkeit und anderen Streitbeilegungsentscheidungen auswirkt: Der Europäische Gerichtshof hat einen auf die Schiedsklausel im BIT zwischen der Slowakei und den Niederlanden (1991) gestützten Spruch eines Schiedsgerichts $^{341}$ mit Sitz innerhalb der EU (Frankfurt/Main) als mit der in Art. 267 und Art. 344 AEUV verbürgten Autonomie des EU-Rechts unvereinbar angesehen, ${ }^{342}$ so dass der Bundesgerichtshof diesen ,award“ wegen Fehlens einer gültigen Schiedsvereinbarung aufhob (§ 1059 Abs. 2 S. 1 lit. a] ZPO). ${ }^{343}$ Ob dies auch für die Konstellation eines BIT mit Drittstaaten bzw. einem Schiedsort außerhalb der EU (wie bei ICSID) ebenso bewertet würde, erscheint jedenfalls nicht zwingend. ${ }^{344}$

\section{Speziell in Bezug auf Gerichtsbarkeit und Streitbeilegung}

a) „Hochzonen“ von „Hoheitsrechten“

Wenngleich einige europäische Staaten insoweit Vorreiter waren, als sie in ihre (neuen) Verfassungen in der Zeit nach Ende des Zweiten Weltkriegs moderne, differenziertere Regelungen über das Verhältnis zu internationalen Organisationen und speziell zur „Übertragung“ bisher auf nationaler Ebene wahrgenommener „Hoheitsrechte“ auf diese aufgenommen hatten (wie etwa Art. 24 GG 1949 ${ }^{345}$ ), sind angesichts der immer weiter verbreiteten und vertieften Mehrebenenstrukturen in der internationalen (Staaten-)Gemeinschaft solche Bestimmungen heute auch in anderen Kontinenten nicht selten und haben nicht zuletzt afrikanische Länder entsprechende Vorschriften in ihre Konstitutionen einbezogen. Nahezu identisch verlauten die Verfassungen Malis (1992, Art. 117), ${ }^{346}$ des Senegal (Art. 96 Abs. 4)

339 5.5.2020, ABl. EU Nr. L 169 v. 29.5.2020, 1; in Kraft getreten am 29.8.2020 (ABl. EU Nr. 281 v. 28.8.2020,1).

340 In die zwischen EU und Mitgliedstaaten geteilte Zuständigkeit fallen lediglich bloße PortfolioInvestitionen, die Investor-Staat-Streitbeilegung sowie darauf bezogene andere Regelungen, EuGH, 16.5.2017, Gutachten 2/15, Rn. 78 ff. (zum Freihandelsabkommen der EU mit Singapur 2015).

341 Achmea B.V. (formerly Eureko B.V.) v. The Slovak Republic (I) (PCA Case No. 2008-13), 7.12.2012, https://www.italaw.com/sites/default/files/case-documents/italaw3206.pdf.

342 Slowakische Republik v. Achmea, Rs. C-284/16, Urt. v. 6.3.2018, Rs, auf Vorlage des BGH, Beschl. v. 3.2.2016, I ZB 2/15, ECLI:DE:BGH:2016:030316BIZB2.15.0.

343 Beschl. v. 31.10.2018, I ZB 2/15, ECLI:DE:BGH:2018:311018BIZB2.15.0; krit. Reuter, RaöRV 80 (2020), 379 ff.; Carducci, ICSID Review - Foreign Investment Law Journal 33 (2018), 582 ff.

344 Vgl. BT-Drs. 19/2174 v. 15.5.2018, 3 f. (Bundesregierung, Urteil des Gerichtshofs der EU zu Schiedsklauseln in Investitionsschutzabkommen); ferner CMC v. Mozambique, 24.10.2019, ICSID Case No. ARB/17/23, Rn. 296 ff.

345 Vgl. schon Gramlich, Europäische Zentralbank und Art. 24 Abs. 1 GG, 1979.

346 https://www.constituteproject.org/constitution/Mali_1992.pdf?lang=en. 
oder der Demokratischen Republik Kongo (Art. 217), das jeweilige Land sei befugt zum Abschluss „avec tout Etat africain des accords d'association ou de communauté comprenant abandon partiel ou total de souveraineté en vue de réaliser l'unité africaine“. Freilich bleibt eine solche Ermächtigung, die bis zur völligen Aufgabe der eigenen Souveränität geht und damit auch eine Integration in einen gesamtafrikanischen (Bundes-)Staat erfasst, andererseits sehr pauschal, selbst wenn sie - vergleichbar Art. 24 Abs. 1 GG - auch einen Verzicht auf (und den gleichzeitigen „Transfer“" von) einzelne(n) „Hoheitsrechte“(n)“347 gestattet (anders als dort aber räumlich beschränkt), denn weder werden, wie im 1993 novellierten Art. 23 Abs. 1 GG, ${ }^{348}$ inhaltliche Anforderungen aufgestellt, denen die „übernehmende" Gemeinschaft strukturell genügen muss, noch werden wie in Art. 24 Abs. 2 GG einzelne Sachgebiete besonders genannt oder ähnlich wie bei Art. 24 Abs. 3 GG eine international(isiert)e Streitbeilegung speziell adressiert. ${ }^{349}$ Aus dem jeweiligen Regelungskontext ergibt sich ferner, dass das bei einem solchen ,abandon“ einzuschlagende Verfahren das generell bei völkerrechtlichen Verträgen übliche ist, also nicht von vornherein ein höheres Zustimmungsquorum oder gar eine Volksabstimmung gefordert wird.

b) Grundlagen und Grenzen außergerichtlicher Streitbeilegung

Selbst nachdrückliche Protagonisten einer speziellen internationalen Investor-Staat-Streitbeilegung ${ }^{350}$ lassen aber keinen Zweifel daran, dass internationales Investitionsschutzrecht als Teil des öffentlichen Rechts ${ }^{351}$ und Investitionsschiedsgerichtsbarkeit als Ausübung öffentlicher Gewalt ${ }^{352}$ zu kategorisieren sei, um zu begründen, warum in der Praxis vergleichend dieses Rechtsgebiet maßgeblich $\mathrm{se}^{353}$ und insbesondere dort entwickelte, akzeptierte allgemeine Grundsätze den Kontroll- und Entscheidungsmaßstab bildeten. ${ }^{354}$ Gerade dann wird jedoch die Frage aufgeworfen, wie es sich (allgemein und in je konkreten nationalen Verfassungen) mit einem staatlich-gouvernementalen Justizmonopol bzw. (aus Sicht des auf solche Dienste angewiesenen „Kunden“) einem Justizgewährungs- oder zumindest -gewährleistungsanspruch ${ }^{355}$ verhält, einem Problem, das überdies nicht erst bei der zwangs-

347 Zur rechtlichen Konstruktion des Vorgangs vgl. BVerfGE 22, 293 (296), 37, 271 (279 ff.).

348 Gesetz v. 21.12.1992 (BGBl. 1992 II, 2086), Art. 1 Nr. 1, bezogen allein auf die europäische Integration; dazu BVerfGE 123, 267 (344 ff.).

349 Vgl. schon Gramlich/Conen, ZöR 70 (2015), 779 ff.

350 Zugleich als Schiedsrichter aktiv (etwa im Fall Ramot und Levy v. Bulgarien, ICSID Case No. ARB/18/47); dazu auch BT-Drs. 18/3862 v. 28.1.2015, 5, zu BT-Drs. 18/3729 v. 13.1.2015, $1 \mathrm{f}$. (DIE LINKE, Interessengeleitetes Gutachten zu Investorenschutz zurückweisen).

351 Schill, ZaöRV 71 (2011), 247 (259 ff.); ferner Petersmann, ZaöRV 74 (2014), 753 (782 ff.).

352 Schill, aaaO, $266 \mathrm{ff}$.

353 Schill, aaO, $273 \mathrm{ff}$.

354 Schill, aaO, $277 \mathrm{ff}$.

355 BVerfGE 54, 277 (291), 97, 169 (185), 107, 395 (401 ff.); vgl. etwa Broß, Freihandelsabkommen, einige Bemerkungen zur Problematik der privaten Schiedsgerichtsbarkeit, Jan. 2015, $13 \mathrm{ff}$. 
weisen Durchsetzung gerade von Justizentscheidungen (als Gewaltmonopol i.e.S.) aufgeworfen wird, sondern bereits auf der Stufe des (gerichtsförmigen) Erkenntnis- als erstem, wegweisenden Schritt eines Verfahrens friedlicher Streitbeilegung. Die Judikative wird regelmäßig in Verfassungen wie im Primärrecht supranationaler Organisationen (etwa Art. 19 EUV, Art. $251 \mathrm{ff}$. AEUV) als eigenständige, von Rechtsetzung/Legislative und Exekutive getrennte „Gewalt“ aufgeführt (z.B. Art. 1 Abs. 3, 20 Abs. 3 GG), und ihrer Organisation wie den jeweiligen (Rechtsprechungs-)Aufgaben ist durchweg ein eigener Teil (Titel, Kapitel) gewidmet. Vielfach werden die verschiedenen Gerichtszweige oder (oberste) Instanzen explizit genannt; die nähere Ausgestaltung der (staatlicher oder supranationalen) Gerichts„Verfassung“ obliegt sodann dem (parlamentarischen) Gesetzgeber. In dieser generellen Konzeption sind größere Unterschiede zwischen modernen Verfassungsstaaten nur im Detail erkennbar, etwa im Verhältnis von Art. 92 ff. GG zu Art. 116 ff. der Verfassung von Niger (2010) $)^{356}$ oder Art. 67 ff. der gabunischen Konstitution (2018). ${ }^{357}$ Damit handelt es sich freilich um eine originäre und genuine Staats- bzw. Hoheitsfunktion, die wegen dieser Rechtsnatur nur bedingt, partiell bzw. nicht in ihrem Kern auf Private übertragen werden oder von diesen ausgeübt werden darf. Eine „Privatisierung“ der Streitbeilegung ist vielmehr allenfalls dann mit dieser prinzipiellen Zuordnung vereinbar, wenn beide bzw. alle Betroffenen/Beteiligten eine außergerichtliche, abschließende Klärung ihrer (rechtlichen) Meinungsverschiedenheiten aus freien Stücken anstreben, weil hier die regelmäßig grundrechtlich verbürgte Privatautonomie eingreift, welche auch insoweit staatlicher Einmischung in die Lösung privater Angelegenheiten sowohl dem Grunde („ob“) als auch dem Umfang (,wie“) nach Schranken setzt. ${ }^{358}$ Ein öffentliches Interesse an friedlicher Lösung von Streitigkeiten ist trotz deren formeller Begrenzung auf die jeweiligen Streitparteien stets vorhanden (und zeigt sich nicht zuletzt im Prinzip der Öffentlichkeit des Verfahrens, Art. 6 Abs. 1 EMRK, ${ }^{359}$ Art. 14 Abs. 1 UN-Pakt über bürgerliche und politische Rechte ${ }^{360}$ ); jedoch zwingt dieser Umstand nicht stets zum Einsatz staatlichen Personals oder öffentlicher (finanzieller) Ressourcen, weil ein faires, neutrales, unabhängiges Vorgehen mit einem gerade wegen solch“ organisatorisch-prozeduraler Legitimation ,guten“ Ergebnis auch auf andere Weise sichergestellt werden kann. Daher (und dann) stellt auch Verfassungsrecht Mechanismen einer „alternative dispute resolution“ kein generelles Hindernis in den

356 https://www.constituteproject.org/constitution/Niger_2017.pdf?lang=en, Fassung 2017.

357 Loi No. 001/2018, http://www.ilo.org/dyn/natlex/docs/ELECTRONIC/109499/135858/F8727439 88/GAB-109499.pdf.

358 Allgemein etwa Zhang, Liberalisierung, Internationalisierung und Renationalisierung in den Rechtsreformen der Schiedsgerichtsbarkeit, 2012, 31 ff.; ferner Krajewski und Schill, EuZW 2015, 105.

359 https://www.echr.coe.int/Documents/Convention_deu.pdf; vgl. Handbuch zu den europarechtlichen Grundlagen des Zugangs zur Justiz, 2016, $51 \mathrm{ff}$.

360 https://www.ohchr.org/Documents/ProfessionalInterest/ccpr.pdf. 
Weg ${ }^{361}$ kommen sie nicht zuletzt auch bei Handelssachen und zwischen privaten, mündigen bzw. geschäftserfahrenen Rechtspersonen (wirtschaftliches Handeln des Staates „wie“ ein Unternehmen eingeschlossen) in Betracht und sind daher innerstaatlich wie im grenzüberschreitenden Kontext weit verbreitet. ${ }^{362}$ Anders verhält es sich aber, wenn es um eine unparteiische Kontrolle von Gesetzen oder anderen iure imperii getroffenen Maßnahmen geht, auch innerhalb des gerichtlichen Instanzenzugs. So ist es nur konsequent, wenn auch bei intergouvernemental eingehegten ,investor-State investment disputes“ die Rechtmäßigkeits-Kontrolle gaststaatlicher Hoheitsakte keine Befugnis zu deren Aufhebung umfasst und zudem eine Feststellung von Rechtsverletzungen auch nicht eine Verpflichtung (bzw. Verurteilung) zur Restitution zur Folge hat, sondern lediglich Geld-Schadensersatz durchgesetzt werden kann. ${ }^{363}$ Denn der dem Investor entgegen tretende (Gast-)Staat macht hierbei von seiner (territorialen) Souveränität Gebrauch, auch wenn er dabei völker(vertragsund zudem -gewohnheits)rechtlich reglementiert und beschränkt ist, und agiert auch gegenüber einem ausländischen Investor in einem vertikalen Über-/Unterordnungsverhältnis (wegen und in Bezug auf dessen inländische Investition). Sowohl dieser ,host State“ als auch der mit ihm für „seine“ Investoren ein IIA schließende „,home State“ bleiben auch bei externem, völkervertraglichem Handeln an ihre jeweiligen verfassungsrechtlichen Vorgaben für eine Wahrnehmung (und gegebenenfalls „Auslagerung“) von (gerichtlicher) Hoheitsgewalt gebunden (sowohl wenn dieser Transfer an andere inländische als auch wenn er an ausländische oder intergouvernementale/fremde Stellen erfolgt ${ }^{364}$ ), und sie können sich hiervon nicht über Abschluss und (pflichtgemäße) Erfüllung zwischenstaatlicher Verträge lösen, wenn und soweit nicht die nationalen Verfassungen solche alternative (nicht-richterliche) Streitbeilegung erlauben oder aber diese Normen (außerhalb ihres unantastbaren Kerns) zugleich förmlich geändert werden. Die zentrale Funktion der (klassischen) Judikative verhindert eine Diversifizierung von Mechanismen und einen vermehrten, die Eigenarten jeweiliger Themen und betroffener Personen(gruppen) beachtenden Einsatz nicht- oder vor-gerichtlicher Streitbeilegung allerdings dann nicht, wenn dies den Kern der „dritten“ Staatsfunktion unberührt lässt, und begegnet daher um so weniger Bedenken, je mehr und enger alternative dispute resolution (ADR) allgemein bewährten (fairen, neutralen) Verfahrensmaximen folgt (wozu auch weitgehend, aber nicht gänzlich unbegrenzte Transparenz

361 Classen, EuZW 2014, 611 (614 ff.); aber auch (rechtspolitisch) BT-Drs. 18/3747 v. 14.1.2015, 2, 4 (Antrag BÜNDNIS 90/DIE GRÜNEN, Konsultationsergebnisse beherzigen - Klageprivilegien zurückweisen).

362 In Deutschland Mediationsgesetz v. 21.7.2012 (BGB1. 2012 I, 1577) zur Umsetzung der Richtlinie 2008/52/EG v. 21.5.2008, ABl. EG Nr. L 136 v. 24.5.2008, 3; ferner Verordnung über OnlineStreitbeilegung in Verbraucherangelegenheiten (EU) Nr. 524/13 v. 21.5.2013, ABl. EU Nr. L 165 v. 18.6.2013, 1; Hilgers/Tobisch, Alternative Streitbeilegung, Deutscher Bundestag, Wissenschaftliche Dienste Nr. 20/2017, 17.8.2017.

363 Zu Fällen einer Naturalrestitution Krönke, ZaöRV 76 (2016), 97 (135ff.); ferner Hindelang und Kriebaum, in: Hofmann/Tams (Fn. 113), $161 \mathrm{ff.}, 201 \mathrm{ff}$.

364 Vgl. BVerfGE 58, 1 (27 ff.), 59, 63 (85 ff.). 
zählt ${ }^{365}$ ), persönlich und fachlich kompetente, unparteiische und (sonst) auch vom Verfahrensausgang nicht tangierte Personen als Entscheider oder anders maßgeblich mitwirken und schließlich der Einsatz von Zwangsmitteln zur Durchsetzung gegen säumige Parteien nicht ohne gouvernementale Unterstützung stattfinden kann (und darf). Hier erweist sich das staatliche Gewaltmonopol als Pendant der Friedenssicherungspflicht, ${ }^{366}$ aber rechtfertigt zugleich auch das Verbot der Selbsthilfe und die Reduzierung legitimen, ,außergesetzlichen“ Widerstands auf eine ultima ratio-Konstellation (so Art. 20 Abs. 4 GG). ${ }^{367}$ Zwangsweise Durchsetzung von gerichtlich wie außergerichtlich erzielten finalen Ergebnissen ist ihrerseits nur nach Maßgabe der (Geeignetheit und) Erforderlichkeit der konkret eingesetzten Mittel (also mittelbar der „guten“ Sache selbst) gestattet und bleibt einer gerichtlichen (Fremd-)Kontrolle unterworfen, die jedoch angesichts der arbeitsteiligen Kooperation mehrerer involvierter Stellen nur einen eingeschränkten Umfang zu haben braucht, damit unnötige Verzögerungen oder gar „Endlosschleifen“ vermieden werden. Nur der Vollständigkeit halber erwähnt sei, dass Sitz bzw. Standort einer Streitbeilegungseinrichtung sich wesentlich (und nicht nur ,informell“) auf konkrete Verfahren und deren Resultat auswirken (können), nicht nur im Hinblick auf das jeweilige rechtliche Umfeld, sondern auch auf Institutionen, Fachpersonal, (Rechts-)Sprache ${ }^{368}$ und Kultur. Die Orientierung am franko- oder anglophonen (oder auch einem anderen) Rechtskreis ${ }^{369}$ zeigt sich nicht allein an der jeweiligen Verfahrenssprache, sondern auch bei in bestimmten Rechtsordnungen verwurzelten Termini, Rechtsinstituten und Konzeptionen, die nicht bruchlos ,übersetzt“ werden können, sondern zwangsläufig zu einer erhöhten Bedeutung der Entscheiderpersönlichkeit(en) und deren juristisch-gesellschaftlicher Sozialisation führen.

\section{Komplexität}

Der durchaus funktionierende, aber hoch-, wenn nicht überkomplexe und auch daher reformbedürftige Status Quo wird so gekennzeichnet durch eine (im Hinblick auf die Diversität und Vielzahl von Hauptakteuren auf internationaler Ebene) notwendig vielfältige, aber nicht systematisch geordnete Struktur aus gouvernementalen wie privaten internationalen, gerichtsähnlichen und nicht-richterlichen Streitbeilegungsinstitutionen, deren Effektivität abhängt von der Einbeziehung nationalstaatlicher Gerichte, weniger in der Phase des Er-

365 Grenzen ziehen durch Berufsfreiheit (Art. $12 \mathrm{GG}$ ) verbürgte Betriebs- und Geschäftsgeheimnisse (BVerfGE 115, 205 [229ff.]) sowie das Recht auf informationelle Selbstbestimmung (Art. 2 Abs. 1 i.V.m. Art. 1 Abs. 1 GG, BVerfGE 65, 1 [41 ff.], 103, 21 [32f.]) als Rechtfertigung von Vertraulichkeit.

366 Vgl. etwa BVerfGE 69, 315 (355 ff.).

367 Dazu BVerfGE 5, 85 (377), 123, 267 (339).

368 Vgl. den Vorschlag zu $\S 114 a-114 c$ GVG (Kammern für internationale Handelssachen), mit Englisch als weiterer Gerichtssprache neben Deutsch (§184 GVG); vgl. BT-Drs. 19/1717 v. 18.4.2018, 7 ff., 16 f.; Rapp, GVRZ 3 (2020), 2 ff.

369 Vgl. differenzierend Honlet et al., in: Carter (ed.), International Arbitration Review, 11. Aufl. 2020, 1 (5 ff.). 
kenntnisverfahrens als bei der (Zwangs-)Vollstreckung von Entscheidungen gerade auch außerhalb des Gerichtsorts oder jeweiligen Gaststaats. Diese Feststellung gilt auch, ja, gerade für ,international investment disputes“.

\section{Mechanismen und Verfahren bei Beilegung internationaler wirtschafts-, speziell investitionsrechtlicher Streitigkeiten}

\section{Allgemein}

1. Anknüpfung gerichtlicher Zuständigkeit(en)

Wird ein Investor im Staat der von ihm getätigten Investition von (angeblich rechtswidrigen) Maßnahmen dieses Gastlandes betroffen, so stellt sich auch für eine aus einem anderen Staat(sgebiet) kommende Person oder Gesellschaft zuerst die Frage, ob und wie weit denn die örtliche (gaststaatliche) Judikative den Vorwurf der Rechtsverletzung prüfen und entscheiden darf oder muss, denn wenn und soweit solche „local remedies" verfügbar sind, ist andere Abhilfe jedenfalls vorerst rechtlich ausgeschlossen. ${ }^{370}$ Dem entspricht, dass eine internationale Zuständigkeit gaststaatlicher Gerichte an eine reale Aktivität auf dessen Ge-

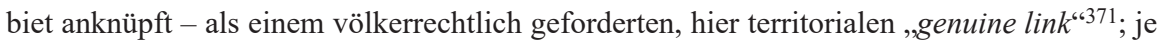
nach Art und (Rechts-)Form einer Investition kommt noch ein Eingreifen von ,personal jurisdiction “372 hinzu. Nach der Gastlandsrechtsordnung ist danach die örtliche, sachliche oder Gerichtszweig-Zuständigkeit zu beurteilen - und auch, ob gegen bestimmte Staatsakte (etwa der Legislative) überhaupt eine (zumindest verfassungs)gerichtliche Überprüfung eröffnet ist. Wenn dies - und sei dies auch nur in begrenztem Umfang bzw. anhand eines beschränkten Maßstabs - tatsächlich der Fall ist, dürfte der Umstand, ob das Justizwesen hinreichend effektiv, insbesondere unabhängig gegenüber den beiden anderen Staatsgewalten funktioniert, erst hernach, unter dem Aspekt einer „Rechtsschutzverweigerung“ (denial of justice $^{373}$ als etwaiges staatliches Fehlverhalten Bedeutung erlangen; lediglich offensichtlich funktionsuntaugliche gerichtliche Rechtspflege mag bereits per se einen Verzicht auf die (Zugangs-)Voraussetzung einer Erschöpfung eines (gast)staatlichen Rechtswegs durch internationale Stellen rechtfertigen.

\section{Abschichtung mehrerer internationaler Zuständigkeiten}

Zwar könnte eine internationale Zuständigkeit stattdessen auch im Hinblick auf die Gerichtsbarkeit im Heimatstaat eines Investors (bzw. dem Land der Errichtung oder des

370 Oben, bei Fn. 210 - 212; zum Aspekt der „,admissibility“ Nolan et al., in: Legum (Fn. 88), 51 ff.

371 BGH, 13.10.2004, I ZR 245/01, JZ 2005, 298 f(f.).

372 In Bezug auf die Staatsan-/-zugehörigkeit des Investors.

373 Oben, bei Fn. 211; ferner Philip Morris v. Uruguay, 8.7.2016, ICSID Case No. ARB/10/7, Rn. $483 \mathrm{ff}$. 
Hauptsitzes eines investierenden Unternehmens) vorliegen, ${ }^{374}$ sogar Anknüpfungspunkte für die Justiz in dritten Staaten könnten sich aus dessen Tätigkeiten dort ergeben, jedenfalls bei sachlichem Zusammenhang mit dem relevanten ,investment “ ${ }^{375}$ Hierfür stellt allerdings Völker(gewohnheits)recht hohe Hürden auf: Ein Staat, der sich das Handeln der Organe der eigenen Judikative zurechnen lassen muss, kann und darf Hoheitsakte eines fremden Staates nicht aufheben, sie nicht einmal zumindest in der Sache überprüfen, sondern hat solche acts of State schlicht als tatsächliche Gegebenheit hinzunehmen. ${ }^{376}$ Allerdings besteht eine solche Ausnahme nur für Handeln iure imperii, nicht auch dann, wenn der beteiligte Staat (angeblich) privatrechtlich-kommerziell, also iure gestionis (und nicht als „Sta-

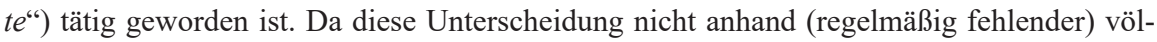
kerrechtlicher Maßstäbe erfolgen kann, stellt sich umgehend die bis heute kontrovers beantwortete Frage, welches nationale Recht denn für die jeweilige Qualifizierung bestimmend sei. ${ }^{377} \mathrm{Ob}$ ein vor ausländischen Gerichten verklagter Staat (oder ein anderes Völkerrechtssubjekt) sich auf einen solchen Prozess einlässt oder gar explizit auf Immunität verzichtet, ${ }^{378}$ ändert im Ergebnis nichts; auch bei einer Verteidigung unter Berufung auf Staatenimmunität müsste (lediglich in einem früheren Stadium) ebenfalls geklärt werden, ob diese, da diese nicht absolut, sondern relativ ist, zu Recht geltend gemacht wird. Damit sind die Aussichten einer im Ausland investierenden Person, vor Gerichten ihres Heimatstaates zu obsiegen, jedenfalls bei typischen ,investment disputes“ letztlich minimal, selbst wenn seine Vermögensposition im Gastland auf Grund eines ,contract" mit diesem erlangt worden ist, wenn und weil dessen Beendigung keine normale Kündigung, sondern einen (wirtschafts)politisch motivierten Eingriff darstellt. ${ }^{379}$

\section{Ungleichbehandlung von Investoren aufgrund der Nationalität?}

Die unterschiedlichen Probleme bei Befassung je zuständiger (inner-)staatlicher Gerichte belegen, dass Institutionen und Verfahren zur Gewährleistung subjektiver Rechte im Einzelfall insbesondere für nicht-souveräne ausländische Streitparteien durchaus sinnvoll sind. Rein tatsächlich führt die Investitionstätigkeit innerhalb eines bestimmten (staatlichen) Territoriums dazu, dass diese örtlich radiziert ist und demzufolge zunächst umfassend (rechtlich) in die Gastlands-Kompetenz fällt, weil diese sich auf alle im Gebiet anwesenden und vor allem ansässigen Personen erstreckt. Soweit weiterhin Menschen- und nicht nur auf die eigenen Staatsangehörigen abstellende Bürgerrechte im Völker- und internen Verfassungs-

374 Als Verbindung von personalen und territorialen ,links“.

375 Entweder wegen eines territorialen Bezugs oder nicht nur unerheblichen von der Tätigkeit ausgehenden Wirkungen (,effects").

376 Vgl. etwa Alderton, Melbourne Journal of International Law 12 (2011), 1 ff.; Akthar, British Journal of American Legal Studies 7 (2018), $205 \mathrm{ff}$.

377 Vgl. BVerfGE 64, 1 (23 ff.), BVerfG, 17.1.2014, 2 BvR 736/13, Rn. 19 ff.

378 Vgl. BVerfGE 117, 141 (152 ff.).

379 Oben, bei Fn. 177, 205. 
recht verbürgt werden, stellt jede Unterscheidung aufgrund der Herkunft zwar noch kein spezifisches Kriterium für (Nicht-)Diskriminierung dar, ${ }^{380}$ jedoch ist auch eine Besserstellung in Bezug auf (fremde) ,nationality“ nur rechtmäßig, wenn dafür (hinreichende) sachliche Gründe vorliegen. ${ }^{381}$ Generell vorhandene oder mutmaßlich nicht nur in Einzelfällen auftretende Rechtsschutzdefizite in einem anderen Staat wären daher nur dann relevant, wenn sie speziell oder in höherem Ausmaß ,foreign investors" betreffen. ${ }^{382}$ Wenn und solange die Zulassung einer ausländischen Investition bzw. der Eintritt eines fremden Unternehmens in den inländischen Markt nicht völker(vertrags)rechtlich gewährleistet wird, sondern eine Entscheidung des Gastlands nach Maßgabe innerstaatlichen eigenen Rechts bleibt (egal ob durch Gesetzes- oder Verwaltungsakt oder als „contract“ mit dem Investor), ist insoweit das allgemeine Investitionsklima maßgeblich und stehen der jeweilige Wirtschaftszweig samt dessen volkswirtschaftlich-gesellschaftlicher Bedeutung im Vordergrund, nicht die konkrete Person des Investors oder die (ausländische) Quelle der investierten Mittel. Wenn und soweit überdies bei der Behandlung von Investor und Investition für die gesamte Lebensdauer im Inland bestimmte Förder- und Schutzstandards zuerkannt werden, so entsprechen diese im Großen und Ganzen den nicht auf Inländer beschränkten, grundrechtlichen Garantien für Eigentum, Leben und Gesundheit („Sicherheit“) und dem allgemeinen Diskriminierungsverbot. ${ }^{383}$ Insbesondere im EU-Rahmen wird aus diesem Grunde schon längst eine im Hinblick auf eine durch personell auf fremde Unionsbürger oder -unternehmen fokussierende Anwendung wirtschaftlicher Grundfreiheiten entstehende „Inländerdiskriminierung “ 384 als durch Heranziehen mitgliedstaatlicher Grundrechte zu kompensierende (Schief-)Lage gewertet, und umgekehrt sind nationale Verfassungsgerichte bestrebt, durch erweiternde, unionsrechtskonforme Interpretation der Staatsverfassung Bürger und Unternehmen aus anderen EU-Ländern den eigenen gleichzustellen, z.B. als „Deutsche“ (Art. 116 Abs. 1) bzw. „,inländische“ juristische Personen (Art. 19 Abs. 3 GG) zu behandeln. ${ }^{385}$ Bis sich eine solche ,integrative“ Sichtweise allerdings über Europa hinaus festigt und letztlich ausdrücklich in Rechtsvorschriften ihren Niederschlag findet, bleiben intergouvernementale wie andere Mechanismen zur Beilegung grenzüberschreitender (Investitions-)Streitigkeiten schon mangels gleichwertiger Alternativen notwendig. Soweit jedoch schon heute (in zulässiger, wenn nicht gar gebotener Weise) Staaten zum einen zu einem effektiven Funktionieren des Erkenntnisverfahrens beitragen (etwa bei Bestellung von Schiedspersonen oder durch technische Unterstützung), zum anderen eine wirksame Um-

380 Vgl. nur Art. 3 Abs. 3, Art. 33 i.V.z. Abs. 1 GG; zur „sozialen“ Anknüpfung von „Herkunft“ vgl. BVerfGE 9, 124 (128f.).

381 Vgl. BVerfGE 78, 179 (196f.).

382 Zum Menschenrecht auf effektiven Rechtsschutz aus Art. 19 Abs. 4 GG vgl. BVerfGE 35, 382 (401); zu Art. 6 EMRK EGMR, 27.7.2006, No. 10523/02.

383 Oben, B.IV.

384 Vgl. BVerfG, 15.12.2016, 2 BvR 222/11, Rn. 47, ECLI:DE:BVerfG:2016:rk20161215.2bvr022211.

385 BVerfGE 129, 78 (94 ff.). 
und Durchsetzung von Schiedssprüchen wie von Resultaten anderer ADR-Verfahren auf nach wie vor Staaten vorbehaltene Mechanismen und Mittel angewiesen ist, hier jedoch der jeweilige ,ordre public“ nicht uneingeschränkt regional, kontinental oder gar global harmonisiert werden kann, muss gerade im Rahmen von ,enforcement“ ebenfalls nationales (Verfassungs-)Recht für angemessenen (Grund-)Rechtsschutz der Streitparteien (wie anderer wesentlich Betroffener) Sorge tragen. ${ }^{386}$

\section{Internationale private und intergouvernementale Streitbeilegung (ISDS) im Vergleich}

1. Strukturelle Gemeinsamkeiten und Unterschiede

Bevor einige wichtige Details mit Fokus auf EU/deren Mitgliedstaaten bzw. Unionsbürger/-unternehmen einerseits und afrikanische, primär OHADA-Staaten als Gegenpartei(en) näher beleuchtet werden, scheint es erforderlich, strukturelle und systematische Aspekte vorweg darzulegen.

Von anderen staatlichen Einrichtungen funktionell getrennte Gerichtssysteme, in deren Rahmen Rechtsstreitigkeiten zwischen verschiedenen Personen durch fachkundige, neutrale, aus öffentlichen Haushalten finanzierte Dritte abschließend entschieden werden, sind heute in allen staatlich organisierten Gemeinwesen anzutreffen, auf internationaler Ebene hingegen nur punktuell in mehreren RECs, auch in Afrika. ${ }^{387}$ Dabei sind für Staat-StaatStreitigkeiten (auch im Hinblick auf grenzüberschreitende Investitionen) regelmäßig ad hoc-Verfahren vorgesehen und es fehlt an einer allgemeinen oder sogar obligatorischen (schieds)gerichtlichen Institution, vor der intergouvernementale Meinungsverschiedenheiten rechtsförmlich ausgetragen werden könnten. ${ }^{388}$ Werden jedoch Staaten selbst oder mittels (formal verselbstständigter) öffentlicher Unternehmen wirtschaftlich wie Private bzw. als Marktteilnehmer tätig, so werden Streitigkeiten mit anderen (Gast-)Ländern nicht mehr als zwischenstaatliche, sondern als kommerzielle oder als ,,investment disputes“ eingeordnet. ${ }^{389} \mathrm{Je}$ nach dem hierfür maßgeblichen (völkervertraglichen oder innerstaatlichen) „Pflichtenheft“ können sowohl Unternehmen als auch (Gast-)Staaten auf Kläger- wie auf Beklagtenseite stehen, wenngleich in der Vergangenheit mangels justiziabler (strikter) Pflichten von ausländischen Investoren in der Mehrzahl der Fälle ein Staat oder dessen Einrichtungen verklagt wurde; ${ }^{390}$ allerdings sind des öfteren Widerklagen (,,counterclaims“)

386 Vgl. Schlussanträge GA'in Kokott, 25.2.2016, Rs. C-559/14, Rn. 41 ff.

387 Insbesondere OHADA, ECOWAS; unten, D.IV.

388 Tanaka (Fn. 191), 24.

389 Etwa die ICSID-Verfahren zwischen Vattenfall v. Deutschland, (oben, Fn. 194, und Case No.ARB/09/06, ,award“ mit ,settlement" agreement, 11.3.2011).

390 Jüngst etwa Genesu S.p.A. v. Ägypten, ICSID Case No. ARB/20/45; Eni International B.V. et al. v. Nigeria, ICSID Case No. ARB/20/41; Qatar National Bank v. Republik Südsudan und Bank of South Sudan, ICSID Case No. ARB/20/40. 
ausdrücklich vorgesehen. ${ }^{391}$ Praktisch ausgeschlossen ist derzeit ein Investitions-Streitbeilegungsverfahren nur zwischen mehreren Unternehmen, weil in diesem Verhältnis auch beim Konflikt zwischen aus- und inländischen Organisationen kein dem Gaststaat zurechenbares (Fehl-)Verhalten relevant werden dürfte. Staat-Investor- als Untergruppe von Investor-Staat-Streitbeilegung erübrigt sich dann, wenn die jeweilige staatliche Partei ihre Rechtsposition durch eigene Legislativ- oder Administrativakte behaupten und durchsetzen kann; hier kommt zunächst eine interne gerichtliche Kontrolle der Verfassungsmäßigkeit in Betracht, die aber regelmäßig nur auf ein diesbezügliches Vorgehen des Betroffenen/Investors hin erfolgt. Auch die eigentliche Vollstreckung ist nur auf dieser Ebene, durch nationale Gerichte überprüfbar und nötigenfalls zu stoppen. Schließlich rufen Rechtsstreitigkeiten bereits vor einer (gerichtlichen) Entscheidung und auch durch deren jeweiligen Inhalt externe Effekte, auch negativer Art, bei anderen Personen(gruppen) als den Streitparteien hervor, seien es Arbeitnehmer, Wettbewerber, (andere) Geschäftspartner/Kunden, einzelne Bevölkerungsgruppen und schließlich das (lokale, nationale, oft aber auch internationale) Publikum im Hinblick auf ,globale (öffentliche) Interessen“ oder „gemeinsame Güter“. Um solche „stakeholder" frühzeitig einzubinden, ohne aber die Grundstruktur einer (schieds)gerichtlichen Entscheidung zu desavouieren, sind verschiedene Optionen verfügbar - eine (fakultative) Partizipation an ,fremden“ Verfahren, mit der Einflussnahme auf deren Ablauf ermöglicht wird, aber auch eine Bindung an die dort getroffene Entscheidung einhergeht, ${ }^{392}$ die Einräumung treuhänderischer Klagerechte an sich für bestimmte Kollektivinteressen engagierende (private) Vereinigungen ${ }^{393}$ oder, wie auf internationaler Ebene des Öfteren, die Öffnung des Erkenntnisverfahrens für tatsächliches oder rechtliches Vorbringen Dritter (Unternehmen, Verbände, Private, weitere Personen), denen eine Rolle als amicus curiae zuerkannt wird, ${ }^{394}$ und nicht zuletzt , third party funding“ “ ${ }^{395}$ Ein zentraler, nicht nur quantitativer Unterschied zwischen außer- (einschließlich schieds-) und gerichtlicher Streitbeilegung ist weniger das Fehlen oder aber Vorhandensein einer Institutionalisierung auf Dau$\mathrm{er}^{396}$ als die Art und Weise sowie Dichte des dafür geschaffenen normativen Rahmens. So legen etwa das ICSID- oder die Gründungsabkommen des PCA lediglich organisatorischstrukturelle Fundamente, um auf der jeweiligen personellen, funktionellen und finanziellen

391 Maritime International Nominees Establishment v Republic of Guinea, 8.1.1988, ICSID Case No. ARB/84/4, auf Basis eines ,investment contract“, nicht eines „treaty“; dazu Naud et al. (Fn. 143); vgl. schon oben, Fn. 193.

392 Im deutschen Zivilprozess Haupt- und Nebenintervention sowie Streitverkündung, §§ 64 ff. ZPO, vor Verwaltungsgerichten Beiladung nach $\S 65$ VwGO.

393 Zivilprozessuale Musterfeststellungsklage, eingeführt durch Gesetz v. 12.7.2018 (BGBl. 2018 I, 1157); § 2 Umwelt-Rechtsbehelfsgesetz (Fassung 2017).

394 Vgl. etwa Levine, Berkeley Journal of International Law 29 (2011), 200 ff.; eingehend Wiik, Amicus Curiae before International Courts and Tribunals, 2017; ferner Segger-Piening, in: Ludwigs/ Remien (Fn. 57), 291 ff.; Born/Forrest, ICSID Review - Foreign Investment Law Journal 34 (2019), $626 \mathrm{ff}$.

395 Unten, F.II.

396 Oben, C.II., III. 
Basis das Agieren von insoweit „unabhängigen“ Einzelpersonen oder Gremien in (diversen) bestimmten Rollen einzuhegen, dem Resultat aber gerade auch rechtliche Entscheidungswirkung beizumessen. Die hierbei tätigen Individuen sind keine Mitarbeiter oder Angehörige der jeweiligen (Träger-)Einrichtung und gehören ebenso wenig (notwendig) dem öffentlichen Dienst eines beteiligten oder dritten Staates an, so dass ihr Aufwand auch anders honoriert wird als bei hauptamtlichen Richtern. Einschlägige fachliche, zumindest auch juristische Qualifikation ist unverzichtbar. Verfahrensöffentlichkeit, Publikation von Entscheidungen und ganz allgemein Transparenz sind bei gouvernementaler Streitbeilegung eng mit den Prinzipien von Rechtsstaatlichkeit und Demokratie verknüpft; ${ }^{397}$ andererseits gibt es auch hierfür Grenzen, die anders als bei (privaten) Unternehmen nicht nur aus dem gebotenen Grundrechtsschutz für Betriebs- und Geschäftsgeheimnisse ${ }^{398}$ resultieren, sondern auch auf wichtige Belange der öffentlichen Sicherheit oder Ordnung gestützt werden können („Staatsgeheimnisse“ unterschiedlicher Gewichtung ${ }^{399}$ ). Streitentscheidung kann außerhalb von (nationalen wie supra- und anderen intergouvernementalen) Gerichten in mehreren, nach Aufgaben und Funktion des Intermediärs gestuften Formen stattfinden, von einer reinen Klärung, ob Tatsachenbehauptungen zutreffen und das Ergebnis festzustellen (fact finding) über die Vermittlung (Mediation i.e.S.) zwischen zwei (oder mehr) unterschiedlichen Seiten (und Dokumentation der dabei erreichten Resultate) und die eine aktivere Rolle des Vermittlers implizierende Schlichtung (conciliation) bis hin zu einem gerichtsähnlichen Verfahren, bei dem jedoch an Stelle hoheitlich bestellter und eingebundener Gerichtspersonen ein oder mehrere Schiedsrichter tätig werden, für deren Entscheidung nicht immer oder zwingend nur rechtliche Maßstäbe wesentlich sind. ${ }^{400}$ Nur bei ,dispute settlement" innerhalb des WTO-Rechts, bei dem es sich aber um ein zwischenstaatliches Verfahren handelt, so dass auch bei investitionsrelevanten Streitpunkten wie im Bereich von GATS oder TRIPS-Abkommen ${ }^{401}$ eine direkte Beteiligung von Investoren nicht vorgesehen ist, ist das von einer Kommission, einem Panel oder dem Appellate Body gefundene Ergebnis noch nicht final, sondern bedarf noch einer Bestätigung durch ein nicht-richterliches (Haupt-)Organ, den Allgemeinen Rat als Dispute Settlement Body; ${ }^{402}$ dies erfolgt jedoch quasi-automatisch, da für das Gegenteil ein „,negativer Konsens“ aller (anwesenden) Teilnehmer dort notwendig wäre. ${ }^{403}$ Die bei außergerichtlicher Streitbeilegung angestrebte größere Flexibilität und Schnelligkeit macht sich aber auch darin bemerkbar, dass eine

397 Gramlich/Conen, SZIER 26 (2016), 391 (413 f.).

398 Oben, Fn. 365.

399 Vgl. §93 StGB (Definition) und §§ 95 ff. (Strafbarkeit verschiedener Arten der Weitergabe an Dritte); dazu etwa BVerfGE 28, 175 (183 ff.).

400 Unten, III.; Tanaka (Fn. 191), 28 ff.

401 Oben, C.II.1.a), II.3.

402 Art. IV Abs. 3 WTO-Übereinkommen (Fn. 216).

403 Vgl. Art. IX Abs. 1 WTO-Übereinkommen, Art. 16.4, Art. 17.14 WTO Dispute Settlement Understanding, Anhang II zum WTO-Abkommen, https://www.wto.org/english/tratop_e/dispu_e/ds u_e.htm. 
Überprüfung von Ergebnissen durch eine übergeordnete (gleichartige) Stelle (auf Rechtsmittel hin) nur unter bestimmten Voraussetzungen und allein im Hinblick auf Rechtsfragen ermöglicht wird; vorgegebene oder üblicherweise vereinbarte kurze Verfahrens- und Entscheidungsfristen ${ }^{404}$ mögen mit dazu beitragen, dass eine zweite umfassende Kontrolle bei intergouvernementalen wie bei privaten Schiedsverfahren nicht in Betracht kommt. Auch im Verhältnis von mehreren intergouvernementalen Gerichten zueinander muss schließlich geklärt werden, welcher Stelle die Letztentscheidung zukommt; der im EU-Primärrecht vorgesehene Beitritt auch der Union zur EMRK ${ }^{405}$ (und damit auch der Verpflichtungen, die dort normierten Vorschriften über die Zuständigkeiten des Europäischen Gerichtshofs für Menschenrechte [EGMR] zu beachten bzw. umzusetzen ${ }^{406}$ ), ist bisher daran gescheitert, dass der Europäische Gerichtshof $(\mathrm{EuGH})$ die ausgehandelte Vereinbarung insoweit für nicht unionsrechtskonform erachtet hat, weil dadurch die eigene in EUV und AEUV ausgestaltete Stellung und Aufgabe beschnitten werde. ${ }^{407}$ Eine durchaus ähnliche (Konflikt-)Konstellation wäre auch auf anderen Kontinenten verträglich zu bereinigen.

\section{Verfahrensgang}

Wesentliche Stufen des Verfahrensablaufs sind vor allem bei „arbitration“ in Anlehnung an staatliche Prozessordnungen aufgebaut und ausgestaltet, teils in Gründungsübereinkommen bzw. einschlägigen nationalen Gesetzen, teils dann in nachrangigen, sekundären Vorschriften der jeweiligen Institution. Zu Beginn geht es durchweg um „admissibility“, wobei auch schon die bindende Option für ein bestimmtes Regelwerk (und damit zugleich der Ausschluss anderer Alternativen) von Bedeutung ist; 408 eine Variante davon ist die in ICSID Arbitration Rules 41, 42 und 44 näher ausgestaltete „bifurcation“. ${ }^{409} \mathrm{Oft}$ findet sich auch als „Filter“ eine Vorabprüfung des Einwandes einer offensichtlichen Aussichtslosigkeit, etwa in ICSID Arbitration Rule 41 Abs. 5, was sich weithin mit dem Ziel einer Mini-

404 Das BVerfG leitet aus der persönlichen Unabhängigkeit von Richtern (Art. 97 GG) ab, diesen müsse ,ausreichend Zeit zu einer allein an Recht und Gesetz orientierten Bearbeitung des Falles“ zur Verfügung stehen, 14.7.2016, 2 BvR 661/16, Rn. 20, https://www.hrr-strafrecht.de/hrr/bverfg/ 16/2-bvr-661-16.php.

405 Art. 6 Abs. 2 EUV; vgl. Obwexer, EuR 2012, 115 ff.

406 Vgl. Art. 33, 46 EMRK; Entwurf des Beitrittsabkommens, BR-Drs. 563/11 v. 16.9.2011.

407 18.12.2014, Gutachten Rs. 2/13; strukturell ähnlich wie im Fall Achmea (Fn. 214).

408 Oben, Fn. 370; ferner Ping An Life Insurance Company of China Ltd. v. Belgien, 30.4.2013, ICSID Case No. Arb/12/29, Rn. 112 ff.; Getma v. Guinea, 16.8.2016, ICSID Case No. ARB 11/29, Rn. $110 \mathrm{ff}$.

409 Vgl. etwa ICSID Case No. ARB/12/3, 8.12.2013, Accession Mezzanine Capital L.P. v. Ungarn, https://www.italaw.com/sites/default/files/case-documents/italaw3132.pdf; Carlson, in: Legum (Fn. 88), 61 ff. Im Verhältnis zum CCJA Getma v. Guinea,29.12.2012, ICSID ARB 11/29, Rn. 19 ff., 94 ff. 
mierung von ,frivolous claims ${ }^{6410}$ deckt. Maßgebliche Bestimmungen befassen sich mit „evidence“ und „proof", neben Beweismitteln auch mit Beweislast und Anforderungen an die Qualität eines Nachweises (,standard of proof $f^{\prime 4}{ }^{411}$ Weiterhin sind regelmäßig vorläufige (provisorische) Maßnahmen vor Verfahrensabschluss vorgesehen. ${ }^{412}$ Während die Vorauswahl von (generell) geeigneten, auch „ethischen“ Anforderungen ${ }^{413}$ entsprechenden (Schieds-)Personen primär der Trägereinrichtung obliegt, sind Modalitäten für deren Bestellung und vor allem Ablehnung im konkreten Fall (mitsamt der hierfür relevanten Umstände und Gründe, vor allem Befangenheit ${ }^{414}$ ) wichtig für die Sicherstellung eines fairen Verfahrens und, wenn schon einem bloßen Anschein von Voreingenommenheit oder Parteilichkeit begegnet wird, auch eines für beide Seite akzeptablen Ergebnisses. Der Kontrolle durch das Publikum dienen ein ausgewogenes Verhältnis zwischen Transparenz und notwendige Vertraulichkeit (,confidentiality“) ${ }^{415}$ sichernde Vorschriften. Die dadurch erzeugte Rechenschaft auch gegenüber Wissenschaft und Fachöffentlichkeit hat zudem oft größere „consistency of rulings“ zur Folge, ohne dabei (mangels rechtlicher Basis) zu einer Bindung an ,precedents“ zu führen. ${ }^{416}$ Die häufige Veröffentlichung von Schiedssprüchen, zuweilen mit abweichenden Meinungen, ${ }^{417}$ fördert ebenfalls die rechtliche Konsolidierung. Bislang nur ansatzweise normiert ist ,third-party funding“, 418 angesichts der oft erheblichen Höhe der Verfahrenskosten ein höchst wichtiges Thema, weil damit zum einen erst ein Streitbeilegungsverfahren praktisch möglich werden mag, jedoch zugleich solche Dritten Verlauf

410 Vgl. Potestà/Sobat, Journal of International Dispute Settlement 3 (2012), 137 ff.; Pac Rim Cayman LLC v. Republik El Salvador, ICSID Case No. ARB/09/12, 26.2.2010, Rn. 114 ff., http://icsi dfiles.worldbank.org/icsid/ICSIDBLOBS/OnlineAwards/C661/DC4233_En.pdf.

411 Vgl. Wiebecke und Stojiljkovic/Favalli, in: Legum (Fn. 88), 122 ff., 128 ff. Zur Heranziehung von Experten vgl. Fukunaga, in: Huerta-Goldman et al. (Fn. 322), 134 ff.; zu „discovery“ KaufmannKohler/Bärtsch, SchiedsVZ 2014, 13 ff.

412 Sinclair/Repousis, ICSID Review - Foreign Investment Law Journal 32 (2017), 431 ff.; Fathallah/Weiss, in: Legum (Fn. 88), 88 ff.

413 Vgl. UNCITRAL/ICSID (draft) Code of conduct for adjudicators in investor-State dispute settlement, https://icsid.worldbank.org/sites/default/files/Draft_Code_Conduct_Adjudicators_ISDS.pd f, 1.5.2020; zum ,double hatting“ Nerea/Mutasa, https://africaarbitration.org/2019/09/02/doublehatting-in-international-arbitration-time-to-close-the-revolving-door/; ferner Fach Gómez, Key Duties of International Investment Arbitrators: A Transnational Study of Legal and Ethical Dilemmas, 2019.

414 Vgl. etwa zu B. Cremades Germa v. Guinea, 28.6.2012, ICSID Case No. ARB 11/29, Rn. 32 ff; zu P. Mayer Hope Services vs. Kamerun, ICSID Case No. ARB/20/02, 21.8.2020, Rn. 57 ff.; Ong, in: Legum (Fn. 88), 165 ff.

415 Oben, Fn. 398; unten, III.2.

416 Vgl. bereits oben, B.II.4.

417 Etwa Capital Financial Holding Luxembourg SA v. Kamerun, ICSID Case No. ARB/15/18, 22.6.2017 (https://www.italaw.com/sites/default/files/case-documents/italaw9017.pdf), und „opinion dissidente“ Mourre, 31.5.2017 (https://www.italaw.com/sites/default/files/case-docume nts/italaw9018.pdf).

418 Unten, F.II. 
und Resultat mitbestimmen (möchten und) könnten, dies jedoch kaum den Anforderungen an ein ,fair trial ${ }^{* 419}$ genügen dürfte. Vor allem seit den TTIP-Verhandlungen zwischen der EU und den USA ist schließlich eine in IIAs nicht seltene Regelung kritisch erörtert worden, die durch das jeweilige Abkommen eingerichteten, von beiden Seiten paritätisch besetzten Regierungsausschüssen ${ }^{420}$ eine Befugnis einräumen, im Hinblick auf die Auslegung eines Vertrags Richtlinien (,guidance“) zu formulieren, die dann als anwendbares Recht auch eine konkrete Investor-Staat-Streitbeilegung prägen können. ${ }^{421}$

\section{Legitimität von Sekundär- und Tertiärrecht intergouvernementaler Einrichtungen}

Ebenso wie beim Erlass von internen, aber verbindlichen Rules wirft eine solche Ermächtigung nicht nur die (rechtsstaatliche) Frage nach hinreichender Bestimmtheit auf, wenn und soweit nichts Näheres zu deren Zweck, Inhalt und Ausmaß verlautet. Vielmehr werden auch beide Male gouvernementale Stellen tätig, die selbst nicht direkt demokratisch legitimiert sind, sondern nur über eine mehr oder weniger lange Kette an das (nationale) Parlament oder das (Staats-)Volk zurückgebunden sind. Zudem sind die tätig werdenden (Amts-)Personen Teil der Exekutive, aber anders als bei Regeln in Staatsverträgen oder Gesetzen werden Parlamente mit den von ihnen geschaffenen Rechtsakten nicht befasst, können ihre Anwendung daher auch nicht verhindern. Auf internationaler Ebene kommt hinzu, dass nur punktuell und mit durchweg geringen Kompetenzen mit Parlamentariern oder gar direkt von der Bevölkerung staatlicher Vertragsparteien gewählten Repräsentanten besetzte Organe bestehen, ${ }^{422}$ in Generalversammlungen wie in Räten von intergouvernementalen Organisationen vielmehr Regierungsmitglieder oder (andere) Staatenvertreter zusammentreffen, beraten (regelmäßig nichtöffentlich) und Entscheidungen treffen. Daher besteht kaum Schutz gegenüber nicht evident die übertragenen Aufgaben und Zuständigkeiten überschreitenden ultra vires-Maßnahmen, weil ein auch auf ihrer Grundlage agierendes Streitbeilegungsgremium insoweit keine Überprüfungs-, jedenfalls aber keine Verwerfungskompetenz hat. Erst in einem späteren Stadium, wenn die zwangsweise Durchsetzung von Ergebnissen ansteht, mögen nationale (Verfassungs-)Gerichte den Vorgang anhalten und in-

419 Zu Maßstäben des GG und der EMRK Brunhöber, http://www.zis-online.com/dat/artikel/2010_1 2_510.pdf.

420 Das heißt nur Mitglieder aus dem Bereich der Exekutive beziehungsweise von dieser bestellt.

421 Klärungsbedürftig auch das Verhältnis zu regulatorischer Kooperation (vgl. Antworten der Bundesregierung zu TTIP, BT-Drs. 18/4432 v. 25.3.2015, und zu CETA, BT-Drs. 18/6000 v. 15.9.2015).

422 Insofern mag die EU ein Vorrreiter i.V. zu anderen RECs bilden, zum einen wegen der Direktwahl der Mitglieder des Europäischen Parlaments (Art. 14 EUV, Art. 223 ff. AEUV), zum anderen wegen ihrer Kompetenzen auch bei Außenbeziehungen (Art. 218 Abs. 6, 10 AEUV). Hingegen kennen (nur, aber zumindest) parlamentarische Versammlungen auch ECOWAS (Art. 6, 13 Vertrag 1993) und EAC (,legislative assembly“, Art. 48 ff. Vertrag); zum panafrikanischen Parlament als Organ der Afrikanischen Union http://www.panafricanparliament.org/index.php/about-u s. 
ländischen Stellen eine Mitwirkung hieran untersagen können. ${ }^{423}$ Während bei von gouvernementaler Seite (mit)gestalteten Regelungen ein Mindestmaß an Ausgewogenheit und Richtigkeit durch die Bindung der Verfasser an Recht und Gesetz gewährleistet wird, macht sich bei mit oder zwischen Privaten vereinbarter außergerichtlicher Streitbeilegung ein Manko bemerkbar, das auf der häufig vorkommenden, tatsächlich vorhandenen und rechtlich erlaubten Nutzung von höchst unterschiedlicher wirtschaftlicher Macht beruht. Solches Gefälle kann auch im Verhältnis von großen (transnational agierenden) Unternehmen und Gastländern bestehen und einseitig begünstigende (bzw. den anderen Teil strukturell benachteiligende) Abweichungen von prozeduralen wie materiell-rechtlichen Normal-Regelungen herbeiführen, weil weder Parität noch Symmetrie das Einschlagen eines Mittelwegs bewirken können. Staatliche Gesetzgebung ist meist bestrebt, derartige Defizite durch (halb)zwingendes Recht zugunsten der typischerweise schwächeren Seite zu kompensieren, zieht der Vertrags(gestaltungs)freiheit aber auch (wenngleich weniger harte) Grenzen, wo es um Verträge zwischen Kaufleuten oder auch zwischen Unternehmen und Staaten geht (siehe in Deutschland §310 Abs. 1 S. 1, 2 Bürgerliches Gesetzbuch - BGB), unabhängig davon, ob diese formal dem Privat- oder dem Öffentlichen Recht zugeordnet werden (vgl. $\S 59$ Abs. 1 und Abs. 2 Nr. 4 Verwaltungsverfahrensgesetz - VwVfG). Im deutschen Recht - und nicht nur dort ${ }^{424}$ - gilt auch insofern als Kontrollmaßstab eine unangemessene Benachteiligung der anderen Vertragspartei entgegen „Treu und Glauben“, worunter sowohl intransparente Allgemeine Geschäftsbedingungen (,terms and conditions") fallen (§ 307 Abs. 1 S. 2 BGB) als auch Bestimmungen, die mit ,wesentlichen Grundgedanken der gesetzlichen Regelung, von der abgewichen wird, nicht zu vereinbaren“ sind oder „wesentliche Rechte oder Pflichten, die sich aus der Natur des Vertrags ergeben, so einschränk(en), dass die Erreichung des Vertragszwecks gefährdet ist“" (§ 307 Abs. 2 BGB). ${ }^{425}$ Andererseits bergen Investor-Staat-Verträge ein Kollusionsrisiko, indem Vereinbarungen lediglich die für die Partei unmittelbar wichtigen Belange regeln, jedoch dabei notwendig oder doch wahrscheinlich nachteilig betroffene Personen, etwa mehr oder weniger große Gruppen der Bevölkerung im Gastland, nicht beteiligt sind, so dass zwar formal kein (unwirksamer) Vertrag zulasten solcher Dritten, aber eine Externalisierung von Kosten ${ }^{426}$ (speziell solcher für die Sicherung der Lebensqualität von Beschäftigten oder Menschen in unter Umständen durch deren hergebrachte Kultur und Lebensweise und für deren Erhaltung existenznotwendigen Räumen) erfolgt, wobei dann eine finanzielle Kompensation weder allein noch vorrangig Belastungen in Gestalt physischer und psychischer Beeinträchtigungen Rechnung

423 So BVerfG, 5.5.2020, 2 BvR 859/15 et al., Rn. 229 ff., ECLI:DE:BVerfG:2020:rs20200505.2bvr085915.

424 Vgl. etwa Appenzeller, Die europäische AGB-Kontrolle, 2017; EuGH, 26.4.2012, Rs. C-472/10, Rn. $32 \mathrm{ff}$.

425 Vgl. etwa BGH, 30.6.2020, XI ZR 119/19, Rn. 16 ff., ECLI:DE:BGH:2020:300620UXIZR119.19.0.

426 Dazu https://www.umweltbundesamt.de/daten/umwelt-wirtschaft/gesellschaftliche-kosten-von-u mweltbelastungen\#gesamtwirtschaftliche-bedeutung-der-umweltkosten. 
tragen kann. Insoweit dürfte auch regelmäßig ein fundamentaler Unterschied zwischen grenzüberschreitenden kommerziellen Transaktionen, deren Gegenstand der Vertrieb von Waren oder Dienstleistungen gegen Entgelt ist, und einer Vornahme von raumbeanspruchenden Investitionen vorliegen, ${ }^{427}$ weil die letztgenannten Aktivitäten insbesondere bei Rohstoffgewinnung und industriellen Produktionsanlagen sowohl bezogen auf konkrete Betriebsstätten als auch die jeweilige Wirtschaft und Gesellschaft eines (Gast-)Staates im Hinblick auf Personen wie auf Wertschöpfung (im Inland) erheblich intensiver ausfallen und letztlich eine andere Qualität (im Sinne von Vor- wie von Nachteilen) aufweisen. Dies mag ein zusätzlicher Grund sein, bei internationaler Handelsschiedsgerichtsbarkeit gewonnene Erfahrungen und gängige Praktiken nicht unbesehen auf Streitbeilegungsmechanismen bei ,investment disputes“ zu übertragen.

\section{Besonderheiten einzelner Streitbeilegungsformen und-mechanismen}

\section{Fact finding}

Ein , fact finding"-Verfahren ${ }^{428}$ ist nicht schon im ICSID-Übereinkommen vorgesehen, sondern erst in Anhang D. der 1978 vom Administrative Council verabschiedeten Additional Facility Rules ${ }^{429}$ dieser Einrichtung, durch die dem ICSID-Sekretariat (Art. 9) die Verwaltung (administration) bei Vorliegen einer von drei in Art. 2 der Rules genannten Voraussetzungen auch in Bezug auf dieses spezielle Verfahren übertragen worden ist. Explizit ist der Verwaltungsrat jedoch nur ermächtigt, ,,rules of procedure“ für Einrichtung und Durchführung von „conciliation“ bzw. ,arbitration proceedings“ zu erlassen (Art. 6 Abs. 1 S. 1 lits. b], c] des Übereinkommens), jeweils mit Zwei-Drittel-Mehrheit der Mitglieder (Art. 6 Abs. 1 S. 2). Als weitere Rechtsgrundlage kommt allein Art. 6 Abs. 3 in Betracht, wonach das ICSID-Hauptorgan diejenigen anderen Befugnisse ausüber oder Funktionen wahrnehmen darf, welches es für „necessary for the implementation for the provisions of this Convention" erachtet. Dem Generalsekretär (Art. 10) wird anderereits in Art. 11 lediglich eine Verantwortlichkeit für die „administration“ von ICSID zugewiesen, einschließlich der „,rules adopted by the Administrative Council" (S. 1), und sodann wird er noch mit zwei speziellen Aufgaben (,registrar“, Art. 28 Abs. 3, Art. 36 Abs. 3) sowie Authentifizierung von in Übereinstimmung mit dem ICSID-Übereinkommen ergangenen „arbitral awards“ (Art. 48) sowie einer Beglaubigung von Kopien hiervon (Art. 49) betraut (Art. 11 S. 2). Auch wenn im ICSID-Verwaltungsrat jeder Vertragsstaat mit je einem ,representative“ vertreten ist (Art. 4 Abs. 1), so ist seine Kompetenz für eine den Anwendungsbereich des Übereinkommens in persönlicher und gegenständlicher Hinsicht erweiternde Regelung um so fragwürdiger, als dafür nicht einmal eine (einfache) Mehrheit der Mitglieder, sondern schon der abgegebenen Stimmen ausreichen soll (Art. 7 Abs. 2), selbst wenn das Gremium nur bei Teil-

427 Vgl. Götze, in: Bogaschewski/Götze (eds.), Unternehmensplanung und Controlling, 2013, $165 \mathrm{ff}$.

428 Allgemein oben, II.1.

429 Oben, Fn. 73. 
nahme einer Mitgliedermehrheit beschlussfähig ist (Art. 7 Abs. 3 des Übereinkommens). Wie schon die Bezeichnung erhellt, wird hier einzeln oder gemeinsam eine, inquiry to examine and report on facts" angestrebt (Art. 1), sodann eine einzelne geeignete (s. Art. 7) Person oder ein Komitee mit ungerader Mitgliederzahl eingerichtet (Art. 8 Abs. 1), die nach Schluss des Verfahrens (Art. 15) einen Bericht erstellt (Art. 16). Die Parteien sollen zwar bestmöglich mit dem Komitee zusammenarbeiten (Art. 18), sind jedoch „entirely free as to the effect to be given to the Report" (Art. 17).

\section{Mediation}

"Conciliation" in einem weiteren Sinne, wie dieser bereits im Bericht des Weltbank-Exekutivdirektoriums zum Entwurf des ICSID-Übereinkommens ${ }^{430}$ zum Ausdruck kommt, umfasst jeden Vorgang ,which seeks to bring the parties to agreement", ${ }^{431}$ und bezieht daher auch Mediation $^{432}$ ein. Auch diese Art der Streitbeilegung wird aber nicht in den auf die Konvention zugeschnittenen Conciliation Rules näher ausgestaltet und ebenso wenig im Rahmen der Additional Facility (in Anhang B.). Bisher beschränkte sich die Rolle von ICSID auf das Angebot von „mediation services“ und „,capacity building“ Aktivitäten; seit 2018 läuft allerdings auch eine Erarbeitung von Mediation Rules, ${ }^{433}$ die bislang speziell für Investitionsstreitigkeiten nirgends existieren. Diese sollen ihre Basis (allein?) in Art. 7 des Übereinkommens finden und das Sekretariat ermächtigen ,to administer mediations that relate to an investment, involve a State or a R(egional)E(conomic)I(ntegration)O(rganization), and which the parties consent in writing to submit to the Centre" (draft Rule 2 Abs. 1). Abweichende Vereinbarungen sind in größerem Umfang zulässig (draft Rule 3), sogar von der Festlegung, der Mediator solle ,impartial and independent of the parties“" sein (draft Rule 12), sowie den Bestimmungen über „,conduct of the mediation“ (Kap. V.), wonach der Mediator die Parteien lediglich (,,in good faith and in an expeditious and cost-effective manner") unterstützen solle, um „, a mutually acceptable resolution" des Streits oder einzelner Probleme zu erreichen, aber nicht die Befugnis habe „to impose a resolution of the dispute on the parties" (draft Rule 17 Abs. 1, 2). Schließlich soll der ICSID-Generalsekretär an der „notice of termination“ (draft Rule 22) mitwirken (insbesondere jeder Partei eine beglaubigte Kopie davon übermitteln) oder, wenn kein Mediator bestellt worden ist, diese selbst fertigen. Regeln zur Umsetzung des Ergebnisses werden anscheinend nicht für erfor-

430 Abgedruckt in: ICSID (Fn. 60), 35 ff.

$431 \mathrm{AaO}$, Rn. 37.

432 Oben, II.1.; für ähnliche Regelungen zur Beilegung verbraucherrechtlicher Streitigkeiten vgl. EU-Richtlinie 2013/11 v. 21.5.2013 (ABl. EU Nr. L 165 v. 18.6.2013, 63); zur Grundlage in der Privatautonomie vgl. BT-Drs. 18/5089 v. 9.6.2015, 40, zu Anforderungen betr. Qualifikation, Unabhängigkeit und Unparteilichkeit von Streitmittlern aaO, $55 \mathrm{f}$.

433 Dazu Nitschke, 10.1.2020, http://mediationblog.kluwerarbitration.com/2020/01/10/a-preview-of-i csids-new-investor-state-mediation-rules/; ferner Ubilava/Nottage, https://icsid.worldbank.org/sit es/default/files/amendments/public-input/Ubilava_Notage_10.17.2018.pdf. 
derlich angesehen. Nach Aufbau und Inhalt dürften sich die künftigen ICSID Mediation Rules weitgehend an entsprechenden Regelungen von UNCITRAL, ICC und der International Bar Association (IBA) ${ }^{434}$ ausrichten, die allerdings nicht speziell auf ,investment disputes“, sondern allgemein auf „kommerzielle“ Meinungsverschiedenheiten ausgerichtet sind. Einen weitaus engeren räumlichen Anwendungsbereich (weil auf Mitgliedstaaten der Organisation begrenzt) beansprucht demgegenüber das OHADA-Regelwerk, das in der Sache jedoch denkbar weit gefasst ist; Art. 1 Abs. 1 lit. a) des Acte Uniforme über „arbitrage“ $(2017)^{435}$ schließt jeden „procéssus“, unabhängig von seiner Bezeichnung, ein „dans lequel les parties demandent à un tiers de les aider à parvenir à un règlement amiable d'un litige, $d^{\prime}$ un rapport conflictuel ou d'un désaccord découlant d'un rapport juridique, contractuel ou autre ou lié à un tel rapport, impliquant personnes physiques ou morales, y compris des entités publiques ou des États. " Ausgenommen sind nur Bestrebungen zu gütlicher Einigung innerhalb von Gerichts- oder Schiedsverfahren (Art. 2 S. 2). Wie anderswo auch ist „confidentialité" (nach Art. 10) die Regel (ebenso draft ICSID Rule 10). Explizit normiert sind hier zudem der weitere Verfahrensgang bzw. eine Durchsetzung von Resultaten einer Mediation durch staatliche Stellen bzw. Gerichte (Art. 12, 15, 16).

\section{Schlichtung (conciliation)}

(Außergerichtliche) Schlichtungsverfahren zielen zwar nach einer Klärung der Streitpunkte auf eine Unterstützung der Parteien ab, diese im gegenseitigen Einverständnis beizulegen; der oder die Schlichter(kommission) können aber in jedem Verfahrensabschnitt konkrete „terms of settlement“ empfehlen, verfügen über gerichtsähnliche Untersuchungsbefugnisse und erstellen sowohl bei Erreichen einer Einigung (über diese) als auch bei deren Fehlschlagen einen Bericht, der (bei ICSID durch dessen Generalsekretär als „certified copy", Art. 11 S. 2 des Übereinkommens) den Parteien zugestellt wird. ${ }^{436}$ Neuere Texte widmen sich auch dem „enforcement“. So sieht Art. 17 Abs. 1 des (auch Mediation umfassenden) UNCITRAL Model Law von $2018^{437}$ vor, ,international settlement agreements“ (nach Art. 16) würden durchgesetzt ,in accordance with the rules of procedure" des jeweils mit diesem Verlangen befaßten Staates; Art. 18, 19 führt Voraussetzungen und Gründe auf, warum eine „competent authority“ dieses Staates (also nicht zwingend ein Gericht) es ablehnen kann ,to grant relief“. Insbesondere im Rahmen des ICSID-Übereinkommens und der Ad-

434 IBA Rules for Investor-State Mediation (2012), https://www.ibanet.org/Publications/publications _IBA_guides_and_free_materials.aspx; dazu Nitschke, ICSID Review - Foreign Investment Law Journal 29 (2014), $112 \mathrm{ff}$.

435 Näher unten, IV.1.a).

436 Vgl. ICSID Case No. CONC(AF)/12/2, 12.5.2015, https://jusmundi.com/en/document/decision/fr -republic-of-equatorial-guinea-v-cms-energy-corporation-and-others-pas-encore-disponible-frida y-29th-june-2012.

437 https://uncitral.un.org/en/texts/mediation/modellaw/commercial_conciliation. 
ditional Facility haben mehrfach „conciliation proceedings“ im Hinblick auf afrikanische Staaten stattgefunden, außer Gabun auch Äquatorial-Guinea und Kamerun. ${ }^{438}$

\section{Schiedsgerichtsbarkeit (arbitration)}

Außergerichtliche, aber gerichtsähnliche Streitbeilegung in einem Schiedsverfahren ist ebenfalls nicht allein beim ICSID angesiedelt, ${ }^{439}$ gerade für Investor-Staat-Streitigkeiten ist dessen Regelungs- und institutioneller Rahmen aber der heute wichtigste und bekannteste, zumal hierdurch der zeitliche und finanzielle Aufwand bei der Errichtung von ad hoc-Tribunalen vermieden wird. Die Rolle und Aufgabe von Schiedsrichtern vereint Elemente eines Schlichters und eines Richters: Einerseits finden sich im ICSID-Übereinkommen gemeinsame Regeln für (Mitglieder von) Panels of Conciliators bzw. of Arbitrators (Art. 12 16), den Ort des Verfahrens (Art. 62 f.) sowie ein eigenes Kapitel (V.) über das Verfahren bei „replacement" und „disqualifications" von Personen aus beiden Gruppen (Art. 56 - 58). Übereinstimmungen gibt es zudem bei Bestellung der im Einzelfall tätig werdenden Panelists (Art. 30 f./Art. 38, 40) sowie der alleinigen Entscheidung über die eigene Zuständigkeit (Art. 32 Abs. 1/Art. 41 Abs. 1). Nur Schiedsgerichten sind aber breite und weitreichende Untersuchungsbefugnisse während des Verfahrens zugewiesen, einschließlich der Befugnis, temporäre oder vorläufige Maßnahmen zu treffen (Art. 43, 46f.). In der Regel muss sich ihre Entscheidung, sofern die Parteien sich nicht (noch bzw. anders) einigen, an einem normativen Maßstab ausrichten (applicable law ${ }^{440}$ ), so vage dieser auch sein mag (wie auch bei Art. 42 Abs. 1 des ICSID-Abkommens). Ein (gegebenenfalls mehrheitlich beschlossener) Schiedsspruch muss sich daher mit dem Vorbringen der Parteien auseinandersetzen und (in Erwägung dessen) eine Begründung für das erzielte Ergebnis enthalten, und abweichende (Minderheits-)Meinungen dürfen beigefügt werden (vgl. Art. 48 Abs. 3, 4); hier gleichen die Regeln denen für (oberste) Gerichte, auf nationaler wie internationaler Ebene. Anders als im innerstaatlichen Recht ist eine Veröffentlichung von „awards“ zwar durchweg von der Zustimmung der Parteien abhängig, bei ICSID-Verfahren aber (trotz Art. 48 Abs. 5) fast schon die Regel. ${ }^{441}$ Charakteristisch für Schiedsverfahren (und insoweit wieder gerichtsähnlich) ist schließlich das Eröffnen, wenn auch nur recht eingeschränkt, einer internen Überprüfungsmöglichkeit, die mit unterschiedlichen Zielen verknüpft sein kann, bloße „Auslegung“ oder erneute Behandlung (revision), wenn möglich durch das ursprüngli-

438 Hess Equatorial Guinea, Inc. and Tullow Equatorial Guinea Limited v. Republic of Equatorial Guinea (ICSID Case No. CONC(AF)/12/1); La Camerounaise des Eaux (CDE) v. Republic of Cameroon and Cameroon Water Utilities Cooperation (CAMWATER) (ICSID Case No. CONC/19/1).

439 Vgl. Tanaka (Fn. 191), $346 \mathrm{ff}$.

440 Dazu etwa Schreuer, McGill Journal of Dispute Resolution 2014, $1 \mathrm{ff}$.

441 https://icsid.worldbank.org/news-and-events/news-releases/publication-icsid-decisions-and-awar ds-parties-consent (5.5.2010); auch bei Verfahren nach der Additional Facility, etwa Grupo Contreras S.L. v. Äquatorial-Guinea, 4.12.2015, ICSID Case No. ARB(AF)/12/2. 
che Schiedstribunal (so Art. 50 bzw. Art. 51) oder aber eine Annullierung, wofür jedoch bestimmte einzelne, gravierende Mängel vorliegen (und zunächst einmal geltend gemacht werden) müssen. In ICSID-Verfahren (nach Art. 52) wird hier ein neues, dreiköpfiges Ad hoc Committee tätig. Mündet dieses prozedural nach den Regeln für das Ausgangsverfahren ablaufende in ein „annulment “ ${ }^{442}$ so kann jede Streitpartei die Sache vor ein neu zu bestellendes Tribunal bringen. Nur für „awards“ sind Fragen der Anerkennung und Vollstreckung näher geregelt, weil vor allem die Schnittstelle zur staatlichen (gerichtlichen) Durchsetzung regelungsbedürftig ist; auch diesbezüglich ist das ICSID-Übereinkommen (Art. 53 - 55) ein lehrreiches Beispiel für die wesentlichen Probleme. ${ }^{443}$ Schiedsverfahren enden vielfach ohne bzw. vor einem Schiedsspruch, durch Einigung der Streitparteien oder verschiedene Formen einer ,discontinuance“ (ICSID Arbitration Rules 43 - 45).

Selbst wenn dann eine zwangsweise Durchsetzung mangels „Titels“ ausscheidet, ${ }^{444}$ ist auch dann regelmäßig der Streit beendet, dessen Wiederaufleben allerdings (auch rechtlich) nicht ausgeschlossen. Insoweit zeigen Erfahrungen afrikanischer Staaten eine höchst schillernde Praxis: So hat sich APCL Gambia B.V. rasch mit Gambia verständigt, nachdem der Klage entgegengehalten wurde, sie sei „manifestly without legal merit“ (Rule 41 Abs. 5). ${ }^{445}$ Im Streit zwischen Millicom und Senegal empfahl das Schiedsgericht zwar vorläufige Maßnahmen und bejahte dann seine Zuständigkeit, bestätigte am Ende aber nurmehr die Einigung qua „award““. ${ }^{446}$ Hingegen schien ein ICSID-Schiedsverfahren zwischen BSG Ressources und Guinea ${ }^{447}$ Anfang 2019 beigelegt, in der Zwischenzeit sind aber erneut Gerichte in London und den USA mit der Angelegenheit (insbesondere der Bedeutung angeblicher Korruption) befasst. Einen Annullierungsantrag des Klägers gegen einen PCA-Schieds-

442 Vgl. Cheng, Berkeley Journal of International Law 31 (2013), $236 \mathrm{ff}$; Bottini, ICSID Review Foreign Investment Journal 31 (2016), 212 ff.; zur Abgrenzung gegenüber ,appeal“ vgl. Sacerdoti/Recanati, in: Huerta-Goldman et al. (Fn. 322), $327 \mathrm{ff}$.; als Beispiel (den Annullierungsantrag der Klägerin ablehnend) Capital Financial Holdings S.A. v. Kamerun, 25.10.2019, ICSID Case No. ARB/15/18, https://www.italaw.com/sites/default/files/case-documents/italaw10876.pdf.

443 Unten, E.II.1., III.1.

444 Anders bei Einbeziehung in einen Schiedsspruch nach ICSID Arbitration Rule 43 Abs. 2, so bei Société d'Energie et d'Eau du Gabon and Veolia Africa v. Gabonese Republic and Société de Patrimoine du service public de l'eau potable, de l'énergie électrique et de l'assainissement (ICSID Case No. ARB/18/36).

445 ICSID Case No. ARB/17/40, https://investmentpolicy.unctad.org/investment-dispute-settlement/c ases/853/apcl-v-gambia; Yeo/Yen, in: Legum (Fn. 88), $72 \mathrm{ff.}$

446 Millicom International Operations B.V. and Sentel GSM S.A. v. Republic of Senegal (ICSID Case No. ARB/08/20), 9.12.2009 (provisional measures), 16.7.2010 (Zuständigkeit), https://inves tmentpolicy.unctad.org/investment-dispute-settlement/cases/290/millicom-v-senegal.

447 BSG Resources Limited, BSG Resources (Guinea) Limited and BSG Resources (Guinea) SÀRL v. Republic of Guinea, ICSID Case No. ARB/14/22, https://www.italaw.com/cases/3688; vgl. Sheppard/Bret (Fn. 120). Zum Fortgang https://www.ft.com/content/2cb32a46-12b6-11ea-a2 25-db2f231cfeae; https://www.reuters.com/article/us-vale-sa-lawsuit-idUSKBN22Y2XQ. 
spruch, gestützt auf Art. 1520 des französischen Code de Procédure Civile, ${ }^{448}$ wies der Cour d'appel Paris mangels Aufhebungsgründen zurück. ${ }^{449}$

\section{Regelungen in Afrika, insbesondere im OHADA-Raum}

\section{OHADA}

\section{a) Acte Uniforme zum Schiedsverfahrensrecht}

Schon lange vor Erlass eines einheitlichen Mediationsgesetzes ${ }^{450}$ hat der OHADA-Ministerrat einen Acte Uniforme relatif au droit de l'arbitrage ${ }^{451}$ beschlossen und in Kraft gesetzt; die Bestimmungen wurden dann im selben Jahr (2017) reformiert bzw. ergänzt. ${ }^{452}$ Das aktuelle Regelwerk erfasst jedes (neue, Art. 35) Schiedsverfahren, wenn sich der Sitz des Schiedsgerichts in einem der Mitgliedsländer befindet (Art. 1). Eine von der Wirksamkeit eines Hauptvertrags unabhängige, nicht zwingend der Schriftform bedürfende Schiedsvereinbarung (Art. 3-1, Art. 4) können natürliche und juristische Personen, ferner Staaten, andere Gebietskörperschaften und ,établissements publics“ treffen, soweit es um für sie disponible Rechte geht (Art. 2); die Klausel kann auch normiert sein in einem ,instrument relatif aux investissements“, insbesondere einem „,code des investissements ou un traité bilatéral our multilatéral“" zu diesem Gegenstand (Art. 3). Kap. II befasst sich mit der Zusammensetzung des Schiedsgerichts, das dreiköpfig sein kann, bei fehlender Einigung aber aus einer (natürlichen, geschäftsfähigen) Person besteht (Art. 5); die Mitglieder müssen im Verhältnis zu den Parteien ,impartial et indépendant“ sein und bleiben (Art. 7 Abs. 3). Über eine Ablehnung (récusation) entscheidet gegebenenfalls binnen kurzer Fristen ein staatliches Gericht oder der CCJA (Art. 8). Zu Beginn des Kapitels über das Schiedstribunal (III.) stellt Art. 8-1 nunmehr klar, ob eine etwa vorgeschaltete Etappe zur Einigung erfolglos durchlaufen worden sei. In der Folge werden die je anwendbaren Verfahrensregeln bestimmt (Art. 10, 14), wird die Kompetenz-Kompetenz des Tribunals normiert (Art. 11) und

448 https://www.legifrance.gouv.fr/codes/section_lc/LEGITEXT000006070716/LEGISCTA0000234 27624/\#LEGISCTA000023427652.

449 25.6.2019, https://jusmundi.com/en/document/decision/fr-kontinental-conseil-ingenierie-v-gabon ese-republic-arret-de-la-cour-dappel-de-paris-tuesday-25th-june-2019\#decision_5389.

$450 \mathrm{http} / /$ www.ohada.com/actes-uniformes/1776/acte-uniforme-relatif-a-la-mediation.html.

451 11.3.1999, http://biblio.ohada.org/pmb/opac_css/doc_num.php?explnum_id=480; zum Reformbedarf Dargham/Feigher, Sept. 2016, https://www.nortonrosefulbright.com/en/knowledge/public ations/9318d350/ohada-arbitration-at-the-crossroads.

452 23.11.2017, http://www.ohada.com/actes-uniformes/1767/acte-uniforme-relatif-au-droit-de-1-arbi trage.html; vgl. bereits Gramlich, RiA 2020, 3 (16 f.); zur Änderung Douajni, 11.4.2019,https://gl obalarbitrationreview.com/review/the-middle-eastern-and-african-arbitration-review/the-middleeastern-and-african-arbitration-review-2019/article/recent-developments-in-ohada-arbitration; Niggemann, SchiedsVZ 2020, 22 (26 ff.); Kebe, 8.3.2019, https://afaa.ngo/page-18097/7206108; Terrien, 18.2.2018, http://arbitrationblog.kluwerarbitration.com/2018/02/18/new-ohada-arbitratio n-mediation-framework-glass-half-full/; Bühler, Journal of International Arbitration 2018, $517 \mathrm{ff}$. 
eine Abgrenzung gegenüber staatlicher Gerichtsbarkeit in gleicher Sache vorgenommen (Art. 13). Zur Rechtswahl sieht Art. 15 vor, diese obliege den Parteien; wenn es daran fehle, ziehe das Schiedsgericht die Vorschriften heran, die es für am besten geeignet erachte, gegebenenfalls ,tenant compte des usages du commerce international“ (Abs. 1). Ein Handeln als ,amiable compositeur" bedürfe einer Ermächtigung durch die Parteien (Abs. 2). Statt durch eine „sentence arbitrale“, die in Kap. IV. (Art. 19 ff.) näher behandelt wird (und auch einen Vergleich der Parteien beurkunden kann, Art. 19 Abs. 3), wird das Verfahren in den Fällen des Art. 16 Abs. 3 durch eine ,ordonnance de cloture“ beendet. Nach Art. 18 sind die „délibérations“ des Gerichts „,secrètes“. Ein Minderheitsvotum ist nicht vorgesehen; das Tribunal ist hingegen befugt, den (gegebenenfalls mehrheitlich getroffenen) Entscheid zu interpretieren oder ,erreurs et omissions matérielles qui l'affectent", zu berichtigen (Art. 22 Abs. 2) oder den Spruch auch zu ergänzen (Abs.3), jeweils binnen kurzer Zeiträume (Abs. 4). Kann das Schiedsgericht nicht erneut zusammentreten, obliegt es „à la juridiction compétente de l'Etat partie de statuer" (Abs. 5). Der Schiedsspruch steht einem rechtskräftigen Urteil gleich (,autorité de chose jugée“, Art. 23). Wird dessen vorläufige Vollstreckung begeht, kann das Tribunal diese jeweils durch begründete Entscheidung zulassen oder ablehnen; hernach ergeben sich gerichtliche Zuständigkeiten aus Art. 28. Kap. V. statuiert eingangs in Art. 25 Abs. 1, ein Schiedsspruch ,n'est pas susceptible d'opposition, d'appel ni de pourvoi en cassation". Allerdings kommt nach Maßgabe des Art. 26 und innerhalb der Fristen des Art. 27 ein gegebenenfalls auf Prüfung der Vereinbarkeit mit dem „ordre public international“ beschränkter ,recours en annulation“" vor den zuständigen Gerichten der staatlichen Partei in Frage und in der Folge eine Kontrolle (und gegebenenfalls „cassation“) durch den CCJA (Art. 25 Abs. 2 - 4). ${ }^{453}$ Weiterhin ermöglicht Art. 25 eine „tierce opposition“ jeder Person vor den staatlichen Gerichten, die ohne ,arbitrage“ zuständig gewesen wären, deren Rechte der Schiedsspruch beeinträchtigt (Abs. 5). Vor diese nationalen Gerichte könnte zudem ein „recours en révision“ gebracht werden, wenn das für dessen Erörterung primär zuständige Schiedsgericht nicht mehr agiert; Voraussetzung hierfür ist nach Abs. 6 ,la découverte d'un fait de nature à exercer sur la solution du différend une influence décisive et qui, avant la prononcé de la sentence, était inconnu du tribunal arbitral et de la partie qui demande la révision." Die Annullierung eines Schiedsspruchs stellt grundsätzlich der Einleitung eines neuen Schiedsverfahrens nicht im Wege (Art. 29). Auf Kap. VI. über ,reconnaissance et exécution des sentences arbitrales (Art. 30 - 34) sowie die ergänzenden Bestimmungen im Règlement ${ }^{454}$ (Art. 29 - 31) wird noch unten eingegangen werden.

453 Vgl. bereits Onyema, Arbitration International 26 (2010), 115 (129 f.).

454 23.11.2017, http://www.droit-afrique.com/uploads/OHADA-Reglement-2017-arbitrage-CCJA.pd f. 
b) Regeln zum Schiedsverfahren beim Gemeinschaftsgerichtshof (CCJA)

Ebenfalls vom OHADA-Ministerrat im Jahr 2017 beschlossen wurde ein Règlement d'Arbitrage den CCJA betreffend, ${ }^{455}$ Bezug nehmend auf diesem Gerichtshof in Art. 21 des OHADA-Vertrags zugewiesene Kompetenzen. Art. 1.1 klassifiziert hierbei die zum Zweck „d'assurer la mise en oeuvre et la bonne fin des procédures arbitrales“ wie die „liées à l'examen de la sentence" getroffenen Entscheidungen als „de nature administrative“ (Abs. 2), die zwar zu begründen, aber weder rechtskräftig noch anfechtbar sind (Abs. 7), anders als Zuständigkeiten des CCJA nach Art. 25 des OHADA-Vertrags (Art. 1.2). Gleichwohl enthält das Règlement für beide Verfahrensarten je spezifische Vorschriften (in Kap. II. bzw. III.). Bei der Verwaltung eines Schiedsverfahrens (mit OHADA-Bezug und auch Investitionen betreffend, Art. 2.1) ist es nach Art. 2.2 nicht Auftrag (mission) des Gerichtshofs, selbst einen Streit zu entscheiden, sondern dieser ,nomme ou confirme les arbitres“ (Art. 3 f.), „est informée du déroulement de l'instance“ (nach Maßgabe der Art. 5 ff.; insofern einbezogen in die Regelung zur „confidentialité", Art. 14) und „examine les projets des sentences“ (Art. 22.2); für sein Vorgehen „en matière d'arbitrage“ gilt eine vom Ministerrat genehmigte interne Verfahrensordnung (Art. 2.3). Während des Schiedsverfahrens sind einzelne Entscheidungen vom CCJA zu treffen, insbesondere zu Kosten (Art. 8, Art. 8-1, Art. 11, 24), bei anscheinend vorliegender „absence de convention d'arbitrage“ (Art. 9) und bei fehlender Einigung über den Schiedsort (Art. 13). $\mathrm{Zu}$ den „règles applicable à la procédure“ (Art. 16) bzw. zum ,loi applicable au fond" werden die Vorschriften des Acte Uniforme aufgegriffen; Art. 17 S. 2 präzisiert für letztere: „Dans tous les cas, le tribunal arbitral tient compte des stipulations du contrat et des usages du commerce international.“ Eine „exception d'incompetence" des Schiedsgerichts soll so früh wie möglich vorgetragen werden (Art. 21). Der Schiedsspruch darf erst nach einem ,examen préalable par la Cour“ ergehen (Art. 22) und den Streitparteien übermittelt werden (Art. 25). Eine Vorabkontrolle (die binnen eines Monats durchzuführen ist) gilt außer für endgültige Entscheidungen für solche über die Zuständigkeit und für ,sentences partielles qui mettent un terme à certaines prétentions des parties"; über andere ist der CCJA nur zu unterrichten (Art. 23.1). Nach Art. 23.2 darf der Gerichtshof, ,proposer des modifications de pure forme, attirer l'attention du tribunal arbitral sur des demandes qui ne semblent pas avoir été traitées, sur des mentions obligatoires qui ne figurent pas dans le projet de sentence, en cas de défaut de motivation ou en cas d'apparente contradiction dans le raisonnement, sans toutefois pouvoir suggérer un raisonnement ou une solution de fond concernant le différend." Dieser Zwischenschritt ist auch erforderlich bei ,interprétation, rectification ou complément de la sentence“ (Art. 26 Abs. 7). Für alle nicht explizit im Règlement erfassten Fälle hält Art. 28 sowohl das Schiedsgericht als auch den CCJA an, so vorzugehen, dass ,la sentence soit suceptible de sanction légale“. Die Kompetenzen des Gerichtshofs bei „recours en révision“ (betr. Nomi- 
nierung von Schiedsrichtern) bzw. bei „tierce opposition“ gestalten schließlich Art. 32 und 33 des Règlement näher aus.

\section{ECOWAS Court of Justice}

Die schon im (revidierten) ECOWAS-Vertrag ${ }^{456}$ vorgesehenen (Haupt-)Institutionen Community Court of Justice (Art. 6 Abs. 1 lit. e], Art. 15) und Arbitral Tribunal (Art. 16) werden jeweils näher ausgestaltet durch Protokolle. Der Rechtsakt über den Gerichtshof weist diesem in der Neufassung ${ }^{457}$ von Art. 9 (Nr. 5) die Kompetenz zu ,to act as arbitrator“ iS.v. Art. 16. Der offizielle Internetauftritt des Gerichts besagt insoweit allerdings nur, Draft Arbitration Rules seien dem ECOWAS-Ministerrat zur Prüfung und Genehmigung vorgelegt worden. Bis auf weiteres kann es daher auch trotz der weithin identischen Mitglieder beider Wirtschaftsorganisationen im Verhältnis OHADA und ECOWAS nicht zu Zuständigkeitskonflikten bei der regionalen Schiedsgerichtsbarkeit kommen.

\section{Extraterritoriale Aspekte nationaler Streitbeilegungsverfahren}

Vor allem einige europäische Staaten und auch die EU selbst ${ }^{458}$ verfolgen seit einigen Jahren einen (weiteren) Ansatz, unternehmerisches Verhalten jenseits der eigenen Staatsgrenzen zur Einhaltung gewisser grundlegender (meist menschenrechtlich fundierter) Standards zu verpflichten und zu diesem Zweck zum einen Vorschriften zu schaffen, nach welchen sie im Heimatstaat des grenzüberschreitend tätigen Unternehmens (bzw. dessen [Konzern-]Zentrale) regelmäßig über ihre wirtschaftliche Tätigkeit im Ausland berichten und dabei spezielles Augenmerk auf standardkonformes Verhalten legen müssen. ${ }^{459}$ Darüber hinaus soll für die Prüfung heimatstaatliches Recht (auch prozessual, also eine Zuständigkeit inländischer Gerichte einschließend) gelten und sollen die Unternehmens- bzw. Investorenpflichten nicht nur den je eigenen Organisationsverbund (unabhängig von der näheren juristischen Ausdifferenzierung) erfassen, sondern auch ausländische Geschäftspartner, so dass zumindest bei jeder neuen Geschäftsbeziehung entsprechende Vertragsklauseln vereinbart werden müssten. Schließlich wird auch diskutiert, ob und wie weit die subjektive und objektive Beweislast für korrektes, pflichtgemäßes Verhalten das (Mutter-)Unternehmen treffen soll, wobei eine solche Beweislastumkehr das Risiko, tatsächlich (durch ein Gerichtsurteil) zu Schadensersatz herangezogen werden, wohl beträchtlich erhöhen würde. Gastländer (auch in Afrika) müssten bei Umsetzung eines solchen Konzepts, wenn dies nicht nur in

456 Oben, Fn. 145, https://www.ecowas.int/wp-content/uploads/2015/01/Revised-treaty.pdf.

457 Oben, Fn. 266; vgl. Happold, ICSID Review - Foreign Investment Law Journal 34 (2019), 496 ff.

458 Als Vorarbeit Smit et al., Study on due diligence requirements though the supply chain, Jan. 2020, https://op.europa.eu/de/publication-detail/-/publication/8ba0a8fd-4c83-11 ea-b8b7-01aa75e d71a1/language-en.

459 Dazu auch UNCTAD, Guidance on core indicators for entity reporting on contribution towards implementation of the Sustainable Development Goals, 25.7.2019. 
einzelnen Heimatländern erfolgte, mit insgesamt negativen gesamtwirtschaftlichen Konsequenzen rechnen, wenn und soweit die betreffenden ausländischen (bzw. transnational agierenden) Gesellschaften nicht aufgrund wohlverstandener Eigeninteressen den heimatstaatlichen Forderungen durch Einbindung in die jeweilige „corporate social responsibility“ Rechnung tragen (wollen). ${ }^{460}$ „Legislative jurisdiction“ in Bezug auf Konzernverantwortungs- ${ }^{461}$ oder Lieferkettenregeln ${ }^{462}$ kommt freilich einem Heimatstaat in Bezug auf ,seine“ Unternehmen durchaus zu und wird etwa von den USA seit langem (und überaus extensiv) in Bezug auf das Verhalten von „U.S. persons“ weltweit ausgeübt. ${ }^{463}$ Insoweit stehen auch diverse Lösungswege offen, wie ein von kraft Territorial- bzw. Personalhoheit ergangenen Maßnahmen zugleich, aber in konträrer Weise betroffenes Unternehmen nicht „zwischen zwei Stühlen“ sitzt, sondern weiter erfolgreich wirtschaftlich tätig werden kann und nicht einen der bisherigen Märkte verlassen muss. ${ }^{464}$ Gleichwohl wäre einem Entstehen einer Regelungsvielfalt aus zahlreichen, ähnlichen, aber doch unterschiedlichen Einzelteilen eine Einigung auf internationaler Ebene vorzuziehen, und dies, solange diese nicht in die Form eines multilateralen völkerrechtlichen Vertrags mündet, zumindest in Gestalt eines zwar nicht rechtsverbindlichen, aber trotzdem rechtserheblichen (und damit effektiven) Mechanismus, insbesondere wenn dieser zunächst von einigen „,willigen“ Partnern geschaffen, aber inhaltlich veränderbar und personell erweiterbar angelegt wird. ${ }^{465}$ Eben dies geschieht bei der OECD-Leitsätzen für multinationale Unternehmen ${ }^{466}$ und den hierfür eingerichteten

460 Vgl. EU-Kommission, 6.10.2020, https://ec.europa.eu/germany/news/20201006-globale-lieferket ten_de; Study on directors's duties and sustainable corporate governance, Juli 2020, https://op.eu ropa.eu/de/publication-detail/-/publication/e47928a2-d20b-11ea-adf7-01aa75ed71a1/language-e $\mathrm{n}$.

461 Einfügung eines Art. 101a in die Bundesverfassung der Schweiz, https://konzern-initiative.ch/wp -content/uploads/2020/10/kvi_factsheet_initiativtext_de.pdf; in der Volksabstimmung v. 29.11.2020 in der Mehrheit der Kantone abgelehnt.

462 Vgl. BT-Drs. 19/23829 v. 29.10.2020 (Pläne der Bundesregierung zu einem nationalen Gesetz zu unternehmerischen Sorgfaltspflichten); zuvor etwa BT-Drs. 19/16061 v. 18.12.2019 (Antrag BÜNDNIS 90/DIE GRÜNEN); Rechtsgutachten (Grabosch) zur Ausgestaltung eines Lieferkettengesetzes, https://lieferkettengesetz.de/wp-content/uploads/2020/02/200527_lk_rechtsgutachten _webversion_ds.pdf.

463 Vgl. schon Mann, Schweiz. Juristen-Zeitung 1986, 21 ff.; Kochinke, Computer und Recht 1988, 542 ff.; Quintin, Revue critique du droit international privé 76 (1987), 493 ff. Zu Klagen in den USA wegen Verstoßes gegen das ,law of nations" nach dem Alien Tort Statute, 28 U.S.C. $\S 1350$, s. U.S. Sup. Ct., 17.4.2013, Kiobel vs. Royal Dutch Petroleum, 569 U.S. 108 (2013).

464 Vgl. etwa BT-Drs. 19/22090 v. 3.9.2020 (Bundesregierung, Eckpunkte für ein Sorgfaltspflichtengesetz).

465 Wie beim geplanten Trade in Services Agreement (TISA); vgl. https://ec.europa.eu/trade/policy/i n-focus/tisa/index_de.htm.

466 Oben, bei Fn. $171 \mathrm{f}$. 
Nationalen Kontaktstellen, zumal dort bei Beschwerden über Verstöße Mediationsverfahren vorgesehen sind und auch durchgeführt werden. ${ }^{467}$

\section{E. Umsetzung (enforcement) der Ergebnisse eines Streitbeilegungsverfahrens}

\section{Allgemein}

Gütliche, ohne oder qua (unterschiedlich intensive) Mitwirkung neutraler Dritter zustande gekommene Bereinigungen eines Konflikts (auch im Investor-Staat-Verhältnis) sind dann auch tatsächlich abschließend, wenn die Parteien das Ergebnis akzeptieren und umsetzen, etwa übernommene Pflichten erfüllen oder Maßnahmen korrigieren, und dabei auch Drittbetroffene angemessen berücksichtigt sind, so dass nicht von deren Seite ein scheinbar beendeter Streit neu aufgerollt wird. Verhält sich aber zumindest eine der Parteien tatsächlich oder angeblich nicht dem gefundenen Resultat gemäß, haben die andere Seite und wohl viel weniger noch Dritte selbst die Mittel zur Hand, um Rechtstreue zu erzwingen, wenn und soweit nicht (weiterhin) ein Gegenseitigkeitsverhältnis besteht, so dass das je Geschuldete nur und erst dann geleistet wird, wenn auch das Gegenüber das Gleiche tut. In Betracht kommt solche legale Selbsthilfe am ehesten bei jeweiligen Geldansprüchen und ermöglicht hier oft eine Auf- oder Verrechnung. In allen anderen Situationen stellt sich aber die Frage, ob das jeweilige ,settlement" eine taugliche Grundlage auch einer (Zwangs-)Vollstreckung bilden kann, die jedoch im Hinblick auf das staatliche Gewaltmonopol nicht ohne nach Art und Ausmaß unterschiedlich intensive Mitwirkung staatlicher Stellen, der Exekutive und/ oder der Judikative, möglich ist. ${ }^{468}$ Im Hinblick auf ,investment disputes“ als grenzüberschreitende Streitigkeiten können auch insoweit generell geltende, also möglichst viele Staaten einbeziehende völkervertragliche Festlegungen nützlich sein, da hierdurch (wenn Vertragsparteien sich regelmäßig vertragstreu verhalten) eine Durchsetzung seltener an spezifischen Besonderheiten einzelstaatlichen Vollstreckungsrechts scheitert. Auch für diesen Bereich hat daher das bewährte, weil auch durch vielfältige Rechtsprechung näher konturierte New Yorker Übereinkommen von $1958^{469}$ große Bedeutung; hinzu kommen (neuere) Übereinkommen mit eher regionalem Geltungsbereich, wie die Riyadh-Konvention. ${ }^{470}$ Auf internationaler Ebene lässt sich so vereinheitlichen, ob und wie weit neben Gerichts- auch Schiedsurteile und weitere verbal ausformulierte Ergebnisse außergerichtlicher Streitbeilegungsverfahren ein Gegenstand von Anerkennung (als „Titel“) und weiterer Durchsetzung sein sollen, welche wesentlichen Schritte der eigentlichen Vollstreckung noch vorausgehen müssen und schließlich, ob und wie weit eine Vollstreckung am jeweiligen nationalen und/

467 https://www.bmwi.de/Redaktion/DE/Textsammlungen/Aussenwirtschaft/nationale-kontaktstellenks.html; dazu Krajewski/Bozorgzad/Heß, ZaöRV 76 (2016), 309 (317 ff., 336 ff.).

468 Oben, C.IV.2.b).

469 Oben, Fn. 149.

470 6.4.1983, https://www.refworld.org/docid/3ae6b38d8.html; dazu Onyema, Arbitration International (26), $115(118,128)$. 
oder einem internationalen ,ordre public“ scheitern kann, einschließlich der staatlichen Immunität eines Vollstreckungsschuldners. Denkbar wäre zudem, Regeln für die internationale Zuständigkeit der Behörden des um Mitwirkung ersuchten Staates aufzustellen, zumindest zu fordern, dass ein ,genuine link“ zur Streitsache bestehen müssen bzw. keine „extraterritorial jurisdiction" ausgeübt werden dürfe. ${ }^{471}$

\section{Anerkennung (recognition) und Durchsetzung}

\section{ICSID}

Speziell auf Investitionsstreitigkeiten und Schiedsverfahren zielende Vorschriften enthält zum einen das ICSID-Übereinkommen. ${ }^{472}$ Nach Art. 53 Abs. 1 S. 2 wird, d.h. muss jede Streitpartei „abide by and comply with the terms of the award“ - auch solcher, die Interpretation, „revision“ oder „annulment" betreffen (Art. 53 Abs. 2) - „except to the extent that enforcement shall have been stayed pursuant to the relevant provisions of this Convention"; diese Einschränkung bezieht sich auf Art. 50 Abs. 2, Art. 51 Abs. 4 und Art. 52 Abs. 5, die eine solche Option eröffnen, wenn das Tribunal oder Committee „considers that the circumstances so require“. Gemäß Art. 54 Abs. 1 S. 1 erkennt jede Übereinkommenspartei Schiedssprüche als verbindlich an, die ,pursuant to this Convention“" ergangen sind, also insbesondere den Anforderungen aus Art. 42 und Art. 48 genügen. Zusammen mit der Bundesstaatsklausel in Art. 54 Abs. 1 S. 2 betrachtet, wird dadurch ausgeschlossen, dass der betreffende Staat noch vorbringen kann, das Schiedsverfahren bzw. das Ergebnis sei nicht abkommenskonform; vielmehr steht der Schiedsspruch einem ,final judgment of the courts" in diesem Lande gleich. Art. 54 Abs. 1 S. 1 verpflichtet eine ICSID-Vertragspartei zudem dazu, durch den Schiedsspruch auferlegte ,pecuniary obligations ${ }^{6473}$ durchzusetzen. Für Zwecke von ,recognition“" wie von „enforcement“ ist dem ICSID-Generalsekretär die hierfür zuständige Stelle (Gericht oder andere Behörde) zu nennen, so dass die solche Maßnahmen betreibende Streitpartei informiert ist und dieser Stelle eine (nach Art. 49 Abs. 1) zertifizierte Ausfertigung des Schiedsspruchs vorlegen kann (Art. 54 Abs. 2). Ansonsten bestimmt Art. 54 Abs. 3 lediglich: „Execution of the award shall be governed by the laws concerning the execution of judgments in force in the State in whose territories such execution is sought" (einschließlich der Einhaltung der durch „immunity from execution" gezogenen Grenzen, Art. 55).

471 Hier sind Regeln zu „presciptive jurisdiction“ einschlägig, deren Abgrenzung jedoch umstritten ist und allenfalls im Kern einen Teil des ,ordre international public" bilden mögen.

472 Auch mit Vergleich zum WTO-Verfahren Polásek/Tonova, in: Huerta-Goldman et al. (Fn. 322), 357 ff.; ferner Wolf, in: Ludwigs/Remien (Fn. 57), 255 ff.

473 Speziell zu „,moral damages“ Parish/Newlson/Roesenberg, Berkeley Journal of International Law 29 (2011), 225 ff.; Ehle/Dawidowicz, in: Huerta-Goldman (Fn. 322), 293 ff.; ferner Gaukrodger/ Gordon, Investor-State Dispute Settlement, OECD Working Paper, 2012, 33 ff. 
Voraussetzungen für vorübergehendes ,stay of enforcement" stellt ICSID Arbitration Rule 54 auf; dies wurde etwa Gambia im Hinblick auf den Schiedsspruch im Streit mit Carnegie Minerals Ltd. (Gambia) ${ }^{474}$ gewährt. ${ }^{475}$

\section{OHADA/CCJA}

Ausführlichere Vorschriften in Bezug nicht nur, aber auch auf Investitionen enthält das OHADA-Recht. Das einschlägige Kap. VI. des Acte Uniforme ${ }^{476}$ bestimmt eingangs (in Art. 30), die Zwangsvollstreckung eines Schiedsspruchs erfordere ,une décision d'exequatur rendue par la juridiction compétente dans l'Etat Partie“. Sowohl Exequatur (Zulassung einer „Exekution“) als auch schon die Anerkennung setzen den Nachweis eines Schiedsspruchs voraus (Art. 31 Abs. 1, 2), gegebenenfalls samt einer Übersetzung in eine Amtssprache der staatlichen Partei (Abs. 3), und sind ausgeschlossen ,si la sentence est manifestement contraire à une règle d'ordre public international" (Abs. 4). Für die jeweiligen Verfahren gelten kurze Fristen, nach deren Verstreichen ,l'exequatur est réputé avoir été accordé" (Art. 31 Abs. 5 S. 2). Nur eine Verweigerung des Exequatur unterliegt einer Überprüfung durch den CCJA aufgrund ,pourvoi en cassation“ (Art. 32 Abs. 1). Jedoch umfasst ein „recours en annulation“ bezüglich des Schiedsspruchs innerhalb des Zeitraums der Anrufung der staatlichen Gerichtsbarkeit auch ein Rechtsmittel gegen die das Exequatur gewährende Entscheidung, so dass, wenn die Nichtigkeitsbeschwerde zurückgewiesen wird, nicht nur die Gültigkeit des Schiedsspruchs feststeht, sondern auch die der positiven ExequaturEntscheidung (Art. 32 Abs. 3 und Art. 33). Schließlich verlautet zur Anerkennung von „sentences arbitrales rendues sur le fondement de règles différentes de celles prévues par le présent Acte uniforme“ in Art. 34, diese erfolge „,dans les Etats Parties, dans les conditions prévues par les conventions internationales éventuellement applicables" bzw. bei deren Fehlen unter denselben Voraussetzungen wie den im Acte Uniforme vorgesehenen.

Das Règlement d'Arbitrage des CCJA ${ }^{477}$ weist diesem Gerichtshof in Art. 29 die Kompetenz zu, über einen ,recours en annulation“ $\mathrm{zu}$ entscheiden ,contre une sentence rendue dans un arbitrage de la Cour par un tribunal arbitral" (Abs. 1); Beschwerde- wie Aufhebungsgründe sind gravierende Verfahrensfehler, das Fehlen jeglicher Begründung oder auch eine Unvereinbarkeit mit dem „ordre international public“(Abs. 2). Gegebenenfalls erfolgt eine Verbindung mit einem Exequaturverfahren (Art. 30 Abs. 2 UAbs. 2), und wenn bereits das Schiedstribunal eine vorläufige Vollstreckung seines Spruchs angeordnet hat, kann der Gerichtshof auch hierüber entscheiden (UAbs. 4). Ein Exequatur-Antrag kann (vom Präsi-

474 ICSID Case No. ARB/09/19, https://icsid.worldbank.org/cases/case-database/case-detail?CaseNo $=\mathrm{ARB} / 09 / 19$.

475 Dazu näher Ofolide, März 2019, https://afaa.ngo/page-18097/7210686, und https://afaa.ngo/page$18097 / 7214305$.

476 Vgl. oben, D.IV.1.a); zur ursprünglichen Regelung Onyema, Arbitration International 26 (2010), 115 (120 ff.).

477 Oben, D.IV.1.b). 
denten des CCJA oder einem dazu bestimmten Richter) nur aus ähnlichen Gründen wie bei einer Annullierung abgelehnt werden (Art. 30 Abs. 5); ein Rechtsmittel hiergegen nach allgemeinen Regeln ist nicht ausgeschlossen, wie sich aus Art. 31.1 S. 2 ergibt. ${ }^{478}$ Eine vom Generalsekretär ausgestellte Bescheinigung (,formule exécutoire") ist maßgeblich auch für die je benannte staatliche Stelle (Art. 31.2 i.V.m. Art. 31.1 S. 1). Bei einer Nichtigkeitsbeschwerde gegen einen Schiedsspruch darf der Gerichtshof auch ,au fond“ entscheiden, wenn die Streitparteien dies verlangen (Art. 29.5 Abs. 2); hier ist dann ebenfalls eine ,tierce opposition“ zulässig (Art. 33.1. Satz 2).

\section{Weitere Regelungen}

Art. 29 Abs. 2 der Arbitration Rules des COMESA Court of Justice ${ }^{479}$ verweist bezüglich der ,enforceability“ von ,awards“ (Art. 29 f.) und anderen Entscheidungen (etwa nach Art. 23) lapidar auf Art. 40 des Gründungsvertrags. Für die „execution“ eines Titels, der einer Person ,a pecuniary obligation“ auferlegt, sind danach maßgeblich ,the rules of civil procedure in force in the Member State in which execution is to take place“. Die ,order for execution“ ist der Entscheidung des Gerichts beizufügen ,which shall require only the verification of the authenticity of the judgment by the Registrar" (Art. 41), whereupon, the party in whose favour execution is to take place, may proceed to execution in accordance with the rules of civil procedure in force in that Member State."

Art. 32 des Protokolls über ein SADC Tribunal ${ }^{480}$ erstreckte die dort getroffenen Bestimmungen zu ,enforcement and execution“ auch auf ,disputes“ zwischen ,natural or legal persons" und dieser REC (nach Art. 18), so dass hierdurch im Hinblick auf das SADC Protocol on Finance and Investment (Art. 24) ${ }^{481}$ Schiedsverfahren ebenfalls erfasst würden (Art. 14 lit. c]).

Nach Rule 27 der East African Court of Justice Arbitration Rules ${ }^{482}$ ist ein Schiedsspruch (einschließlich Korrekturen oder Zusätze, Rules 25, 26) ,final“ (Abs. 1), aus der Wahl für ein Schiedsverfahren nach Art. 32 des EAC-Vertrags ergibt sich eine Verpflichtung für die Streitparteien, einen darauf resultierenden Schiedsspruch unverzüglich auszuführen (Abs. 2), und auch hier besagt Abs. 3, die Durchsetzung erfolge ,in accordance with the enforcement procedures of the country in which enforcement is sought."

478 Art. 30 Abs. 4 bezieht sich nur auf die Erteilung; vgl. Niggemann, SchiedsVZ 2020, 22 (25).

479 https://comesacourt.org/wp-content/uploads/2019/11/COMESA-COURT-ARBITRATION-RUL ES-2018.pdf.

480 Fn. 151.

481 https://www.tralac.org/discussions/article/12350-the-first-international-arbitration-case-under-the -sadc-finance-and-investment-protocol-lesotho-v-swissbourgh-diamond-mines-case.html.

482 https://www.eacj.org//wp-content/uploads/2012/08/EACJ_Arbitration_Rules.pdf. 


\section{Einzelfälle}

Für ,commercial awards“ der ICC wie anderer Institutionen ergeben sich trotz vereinzelter weiterer Beitritte zum New Yorker Übereinkommen (so die Demokratische Republik Kongo $2013^{483}$ ) Probleme aus der fehlenden Ratifizierung dieses Vertrags durch viele afrikanische Staaten. ${ }^{484}$ Für ICSID-Verfahren mag die relativ geringe Zahl von BITs bei vielen Ländern bereits ein Zugangshindernis darstellen, bei der Vollstreckung hingegen streitig werden, ob und welche Vermögenswerte als „staatliche“ einem Zugriff unterliegen und wo dieser erfolgen soll. ${ }^{485}$ Probleme können auch die leicht unterschiedlichen Zustellungsvoraussetzungen zwischen New Yorker und ICSID-Konvention (zumindest in nationalen Rechten) aufwerfen. ${ }^{486}$ Generell heikel ist die Frage, wann ausnahmsweise nationale Gerichte eine Unwirksamkeit des Schiedsspruchs bejahen dürfen. ${ }^{487}$ Insoweit hob der Pariser Cour d'appel den Vorrang inter-/supranationalen OHADA-Rechts vor mitgliedstaatlichem Recht (Kameruns) hervor. ${ }^{488}$

\section{Durchsetzung von ADR-Ergebnissen gegenüber staatlichen Streitparteien und durch diese}

\section{Reichweite der Staatenimmunität}

Nur wenn eine staatliche Streitpartei einen Schiedsspruch im eigenen Staatsgebiet durchsetzen will, liegt die Einschaltung inländischer Vollstreckungsorgane einschließlich nationaler Gerichte auf der Hand, wenn und soweit letzteres hierfür nach dem betreffenden innerstaatlichen Recht überhaupt rechtlich notwendig ist. ${ }^{489}$ Umgekehrt sind jenseits des eigenen Territoriums völker(gewohnheits)rechtliche Regelungen zur (fremd)staatlichen Immunität speziell in Bezug auf Vollstreckungsmaßnahmen zu beachten, selbst wenn diese nicht explizit in das jeweilige innerstaatliche Recht einbezogen worden sind (worauf Art. 55 ICSID-

483 Gesetz Nr. 13/023, in engem Zusammenhang zum Erwerb der Mitgliedschaft in OHADA (2012).

484 So Kirtley, Law and Practice of International Courts and Tribunals 8 (2009), 143 (146 ff.); Onye$m a$, Arbitration International 26 (2010), 115 (116ff.).

485 Kirtley, aaO., $153 \mathrm{ff}$.

486 Queen's Bench Division, Commercial Court, 30.6.2020, Case No. CL-2018-00758, (2020) EWHC 1723 (Comm), Rn. $20 \mathrm{ff}$.

487 Vgl. zu Mauritius Nursimulu/Hornan, 7.1.2020, https://afaa.ngo/page-18097/8527897.

488 20.12.2018, No. 16/25484; dazu Barboza, 19.4.2020, http://arbitrationblog.kluwerarbitration.com /2020/04/19/paris-court-of-appeal-upholds-the-supranational-character-of-ohada-law-in-an-action -for-annulment-of-an-arbitral-award/; Kendra et al., 14.2.2019, https://www.hlarbitrationlaw.com /2019/02/the-paris-court-upholds-the-supranational-nature-of-ohada-law-in-dismissing-annulmen t-application-ca-paris-16-25484-20-december-2018/.

489 Regeln über „Verwaltungsvollstreckung“ (wie in Deutschland nach VwVG oder AO) ermöglichen eine zwangsweise Durchsetzung von Geldforderungen und anderen Rechtsansprüchen ohne vorherigen gerichtlichen Vollstreckungstitel. 
Übereinkommen abstellt ${ }^{490}$ ). Ruft allerdings ein Staat selbst die Gerichte eines anderen an, so unterwirft er sich damit zugleich dem Prozess- und letztlich auch dem materiellen Recht des Forumstaates, und überdies dürfte auch für Widerklagen zumindest dann ein ,waiver of immunity $^{\text {“491 }}$ gegeben sein, wenn diese einen (wesentlich) gleichen Sachverhalt (Streitgegenstand) betreffen. Das Recht der Staatenimmunität ist freilich (anders als die Stellung diplomatischer und konsularischer Vertretungen in Ausland ${ }^{492}$ ) bis heute nicht umfassend völkervertraglich geregelt, selbst wenn wohl Konsens herrscht, dass nicht das andere Völkerrechtssubjekt als solches, sondern nur spezifische (gouvernementale) Handlungen von fremdstaatlicher Gerichtsbarkeit ausgenommen wird. Denn damit bleiben zwei zentrale Fragen offen: zum einen, ob und wann auch die Aktivitäten rechtlich verselbstständigter Organisationen (oder zumindest bei Wahl bestimmter Rechtsformen) einem Staat als dessen Tätigkeit zugerechnet werden (müssen), und zum andern, wann eine staatliche Betätigung als iure imperii (hoheitlich) oder aber als iure gestionis (nicht-hoheitlich, ,kommerziell“) zu qualifizieren (und welche Rechtsordnung dafür maßgeblich) ist. ${ }^{493}$

\section{Einzelfälle}

Im Hinblick auf einen ICC-Schiedsspruch gegen Äquatorial-Guinea lehnte so ein U.S. Federal District Court (District of Columbia) den Antrag der zuvor erfolgreichen Partei (Orange Middle East and Africa) bereits deshalb ab, weil eine wirksame Zustellung aufgrund des Foreign Sovereign Immunities Act nicht nachgewiesen sei - und obwohl der Staat einräumte, auf den Immunitätseinwand verzichtet zu haben. ${ }^{494}$ Hingegen erachtete der Londoner High Court of Justice zumindest im konkreten Verfahrensstadium des ,enforcement" den Einwand mangelhafter Übermittlung an Ägypten wegen Nichteinhaltung spezieller Immunitätsregeln nicht für relevant, ${ }^{495}$ während bei einer Vollstreckung eines ICC ,award“ gegen

490 Oben, II.1.

491 Oben, bei Fn. 378; Lorz, Ausländische Staaten vor deutschen Zivilgerichten 2017, 131 ff.; als Bsp. Cour d'appel Paris (Fn. 449), wo sich der beklagte Staat (Gabun) in der Sache einließ. Das Exequatur gehört noch nicht zur Vollstreckung; so Cour de Cassation Paris, 11.6.1991, https://jus mundi.com/en/document/decision/fr-societe-ouest-africaine-des-betons-industriels-v-senegal-fran ce-decision-de-la-cour-de-cassation-chambre-civile-1-tuesday-11th-june-1991\#decision_988, bezogen auf SOABI v. Senegal, ICSID Case No. ARB/82/1 (25.2.1988).

492 Wiener Übereinkommen über diplomatische Beziehungen v. 18.4.1961, Art. 22 ff., Art. 29 ff.; Wiener Übereinkommen über konsularische Beziehungen v. 24.4.1963, Art. 28 ff. Vgl. etwa U.S. District Court (District of Columbia), 16.4.1987, https://jusmundi.com/en/document/decision/en-1 iberian-eastern-timber-corporation-v-republic-of-liberia-us-district-court-for-district-of-columbia -decision-thursday-16th-april-1987, bezogen auf den Schiedsspruch (31.3.1986) LETCO v. Liberia, ICSID Case No. ARB/83/2.

493 Vgl. Schill, in: Hofmann/Tams (Fn. 113), 231 ff.; Mohamed, State immunity vs. enforceability of awards, https://www.iarbafrica.com/en/blog1/480-state-immunity-v-s-enforceability-of-awards.

494 https://www.iarbafrica.com/en/fr/news-list/194-dc-court-dismisses-petition-for-recognition-of-13 4-million-award-against-equatorial-guinea. Zum Schiedsspruch bereits oben, Fn. 253.

495 Oben, Fn. 486. 
Libyen nach dem New Yorker Übereinkommen die Frage nach der generell vorgesehenen Zustellung (über diplomatische Kanäle) und nach Ausnahmen hiervon im Mittelpunkt stand. ${ }^{496}$ Der CCJA legte zunächst Art. 30 des relevanten Acte Uniforme ${ }^{497}$ sehr weit aus, ${ }^{498}$ differenzierte später jedoch und gewährte ,semi-public entities“ (wie konkret den Grands Hotels du Congo) keine Immunität gegenüber Zwangsvollstreckungsmaßnahmen, die Ansprüche aus kommerzieller Tätigkeiten gegenüber Banken betrafen; ${ }^{499}$ anders wurde eine Konstellation behandelt, bei der der Staat alleiniger Anteilsinhaber war. ${ }^{500}$

IV. Fazit

Der Erfolg im außergerichtlichen Erkenntnisverfahren kann sich so letztlich zumindest rechtlich als Pyrrhussieg erweisen, weil weder eine Rechtsverletzung korrigiert noch die dadurch hervorgerufenen finanziellen Aufwendungen und verursachten Schäden ersetzt werden. Dass der säumige Schuldner damit an Reputation verliert und für die Zukunft sowohl als Zielland einer Investition als auch als Kreditnehmer auf internationalen Kapitalmärkten Nachteile zu gewärtigen hat, scheint dabei nicht ganz selten in Kauf genommen zu werden.

Allerdings gibt es auch Konstellationen, bei denen gerade umgekehrt durch Maßnahmen auf (regionaler) internationaler Ebene das Tätigwerden (mitglied)staatlicher Gerichte beeinflusst oder sogar suspendiert wird (etwa nach Maßgabe von Art. 7 EUV ${ }^{501}$ oder auch Art. 258 ff. AEUV ${ }^{502}$ ), wobei in der Praxis aber immer noch offenbleibt, ob ein hiervon betroffener Staat solche rechtliche Intervention hinnimmt oder sich darüber hinwegsetzt. Jedoch sind diese beschränkt auf rechtsstaatliche Defizite der jeweiligen Gerichtsorganisation (einschließlich der Auswahl und Amtsdauer von Richtern) und betreffen nicht konkrete Einzelfallentscheidungen. Hier wird selbst internationalen Menschenrechts-Gerichtshöfen keine Kompetenz zugewiesen, auch die Durchsetzung ihrer gegen eine staatliche Vertragspartei gefällten Urteile zu betreiben (vgl. nur Art. 44, 46 EMRK, Art. 28 - 31 des Protokolls über die Errichtung eines African Court on Human and Peoples‘ Rights ${ }^{503}$ ).

496 Queen's Bench Division, Commercial Court, 3.7.2019, Case No. A4/2019/0283, (2020) EWCA Civ 1110, Rn. $24 \mathrm{ff}$.

497 Portant organisation des procédures simplifiées de recouvrement et des voies d'exécution, 10.4.1998, http://www.ohada.com/actes-uniformes/496/509/livre-2-voies-d-execution.html.

498 7.7.2005, No. 043/2005, Aziablévi Yovo v Togo Telecom.

499 26.4.2018, No. 103/2018, Mbulu Museso case.

500 So Douajni (Fn. 452).

501 Im Gang sind Verfahren gegen Polen und Ungarn, https://www.europarl.europa.eu/news/de/headl ines/eu-affairs/20180222STO98434/bedenken-zur-rechtsstaatlichkeit-in-mitgliedstaaten-was-dieeu-unternehmen-kann.

502 Vgl. EuGH, 5.11.2019, Rs. C-192/18, Kommission v. Polen, ECLI:EU:C:2019:924.

503 https://en.african-court.org/, https://en.african-court.org/images/Basic\%20Documents/africancour t-humanrights.pdf. 


\section{F. Kosten von (Investor-Staat-)Streitbeilegungsverfahren}

\section{Gemeinsamkeiten und Unterschiede im Verhältnis zu Kosten staatlicher Justiz}

Bereits eine Inanspruchnahme staatlicher Gerichte verursacht vielfältige Personal- und Sachkosten, die generell nicht insgesamt von der Bevölkerung als Ganzer getragen und über von dieser zu entrichtende Abgaben refinanziert werden sollten, so dass sie durchweg zu einem mehr oder weniger großen Teil auf die Streitparteien in Form von Gebühren (und Auslagenersatz) übergewälzt werden. ${ }^{504}$ Während insoweit das Verursacherprinzip eine geeignete Grundlage bildet, vermag dieser Grundsatz allein nicht zu rechtfertigen, dass die je unterliegende Partei (im Ausmaß fehlenden Erfolgs) zur Kostentragung herangezogen wird, weil wegen des Justizgewährungsanspruchs ${ }^{505}$ nur ein Kläger, nicht aber auch ein Beklagter in der Lage ist, sich einem Rechtsstreit zu entziehen. Daher müssen Neutralität, Sachkunde und Autorität eines (staatlichen) Gerichts als weitere Argumente hinzutreten, wenn die Kostenentscheidung inhaltlich auf das jeweilige Ergebnis des Verfahrens abstellt (und zudem sogar Kosten einer nur fakultativen rechtlichen Vertretung mit umfasst). ${ }^{506}$ Im Hinblick auf die eigene Judikative kann allerdings staatliche Gesetzgebung bei der Ausgestaltung und Höhe der anfallenden Kosten soziale Aspekte berücksichtigen, vor allem wenn und weil Rechte nur mit gerichtlicher Unterstützung eingefordert werden können, ${ }^{507}$ und angesichts der Masse von Rechtsangelegenheiten bei „Streitwerten“ als Ausgangsgrößen für eine konkrete Berechnung pauschalisieren. ${ }^{508}$ Je nach Disponibilität von Rechten sind auch Regelungen durchaus angemessen, die ein Tätigwerden von Gerichten von der Leistung eines Kostenvorschusses seitens des Klägers abhängig machen. ${ }^{509}$

Bei außergerichtlichen Streitbeilegungsverfahren nehmen die Beteiligten hingegen (auch hier kostenpflichtige) staatliche Unterstützung meist nur am Ende in Anspruch; mit der Abwahl staatlicher Justiz wird die Kostentragung und -verteilung in erster Linie eine allein von den Parteien zu klärende Angelegenheit, was auch hier durch Einsatz von Standardklauseln geschehen kann. ${ }^{510}$ Etwas anders verhält es sich jedoch mit den tatsächlich entstehenden Aufwendungen insbesondere für Schiedsinstitutionen, deren Dienste weit über eine Mitwirkung bei der Bestellung von Schiedsrichtern und technischer Hilfe hinausgehen. Hier setzt etwa im Rahmen von ICSID der Generalsekretär in Umsetzung vom Verwaltungsrat aufgestellter Regeln „,charges“ für die Nutzung von ,facilities“ des Zentrums

504 Für Deutschland etwa $\S \S 91 \mathrm{ff} . \mathrm{ZPO}, \S \S 154 \mathrm{ff}$. VwGO, $\S \S 1 \mathrm{ff} ., \S 17$ GKG.

505 Oben, bei Fn. 355.

$506 \S 91$ Abs. 2 ZPO, § 162 Abs. 2 VwGO.

507 Zur Prozesskostenhilfe ( $\S 114$ ff. ZPO) vgl. BVerfG, 9,11,2017, 1 BvR 2440/16, 2441/16, Rn. 17 ff., ECLI:DE:BVerfG:2017:rk20171109.1bvr244016.

508 Vgl. BVerfG, 8.12.2011, 1 BvR 1393/10, Rn. 4 ff., ECLI:DE:BVerfG:2011:rk20111208.1bvr139310.

509 Vgl. nur $\S \S 12,15,16$ i.V.m. $\S 6$ GKG.

510 Vgl. bereits Gaukrodger/Gordon (Fn. 473), 19 ff.; Flecke-Giammarco, in: Huerta-Goldman et al. (Fn. 322), 389 ff. 
fest (Art. 59 des Abkommens); diese beiden Organe werden gegebenenfalls auch bei der Bestimmung von ,fees" und „expenses“ der Mitglieder von Schlichtungskommissionen oder Schiedsgerichten tätig. Bei ,conciliation“ werden die wesentlichen Kosten gleichmäBig (,equally“) von den Streitparteien getragen (Art. 61 Abs. 1; ICSID Conciliation Rule 8), ebenso bei ,fact-finding proceedings“" (Rule 15); bei „arbitration“" sieht Art. 61 Abs. 2 jedoch mangels abweichender Parteivereinbarung vor, das Schiedsgericht bewerte zunächst die im Zusammenhang mit dem Verfahren eingegangenen Ausgaben der Streitparteien und entscheide dann (im Schiedsspruch), wie und von wem sowohl solche „expenses“ als auch die für Mitglieder des Schiedsgerichts und für Nutzung der Fazilitäten des Zentrum entstandenen Kosten zu bezahlen sind. ${ }^{511}$

Klargestellt werden soll künftig auch, dass Konsequenzen eines „default in payment“ eine Verzögerung oder gar „discontinuance“ des Streitbeilegungsverfahrens sein können (ICSID draft Administrative and Financial Regulation 16 ${ }^{512}$ ). Die UNCITRAL Arbitral Rules stecken den Begriff der „Kosten“ in Art. 40 Abs. 2 näher ab, nennen wesentliche Kriterien, wann ,fees" und „expenses“ für Schiedsrichter „reasonable“ sind (Art. 41), bestimmen als Grundsatz, dass die unterlegene Partei die Kosten zu tragen habe, lassen aber im Einzelfall ,apportionment of costs“ zu (Art. 42) und treffen Regelungen über einen Kostenvorschuss (Art. 43). Ähnlich sind die Regeln (36 - 38) der COMESA Court Arbitration Rules, ${ }^{513}$ wo sich zudem eine Vorschrift zu ,security for costs“ (Art. 39) findet. ${ }^{514}$ Bei OHADA enthält der Acte Uniforme relatif au droit de $1^{\prime}$ Arbitrage ${ }^{515}$ keine Regelung zu Kosten, wohl aber das Règlement des CCJA ${ }^{516}$ in Art. 24: Das Schiedsgericht legt diese nach Maßgabe von Abs. 2 und 3 fest und befindet über die Verteilung auf die Streitparteien (Abs. 1). In einigen kritischen Fällen nimmt der Gerichtshof die Entscheidungen vor (Abs. 4, 5), insbesondere die Höhe von Schiedsrichterhonoraren betreffend.

\section{Drittfinanzierung (third party funding)}

Bei unzureichenden eigenen finanziellen Mitteln können diese von dritter Seite zur Verfügung gestellt werden, damit ein Betroffener eine angestrebte Streitbeilegung überhaupt ein-

511 Vgl. Franck, Arbitrations Costs; Myths and Realities in Investment Treaty Arbitration, 2019; Nagy/Uka, https://africaarbitration.org/2019/09/02/costs-in-international-arbitration-are-changes-nee ded-ola-nagy-orji-uka/.

512 Parallele Regelungen sind geplanr in (Additional Facility) Administrative and Financial Regulations $6-8$.

$513 \mathrm{https} / / /$ comesacourt.org/wp-content/uploads/2019/11/COMESA-COURT-ARBITRATION-RUL ES-2018.pdf.

514 Umstritten für ICSID-Verfahren, vgl. Herzig v. Turkmenistan, 27.1.2020, ICSID Case No. ARB/18/35; Luttrell, Journal of International Arbitration 36 (2019), 385 ff.; vorgesehen in Draft Arbitration Rule 53; ferner Altenkirch/Frohloff, ICSID Review - Foreign Investment Law Journal 33 (2018), $723 \mathrm{ff}$.

515 Oben, Fn. 452.

516 Oben, Fn. 454. 
leiten bzw. zu Ende führen kann; regelmäßig wird dies bei ISDS das betroffene (private) Unternehmen sein. Seitens der Bundesregierung verlautete dazu: „Eine Finanzierung von Klagen als Geschäftsmodell kommt auch bei ordentlichen Rechtsstreitigkeiten vor und ist kein Spezifikum von Investor-Staat-Schiedsverfahren. Eine missbräuchliche Finanzierung, bei der der Geldgeber selbst anstelle der Partei maßgeblichen Einfluss auf die Prozessgestaltung nehmen oder die Partei in ihrer Verfahrensführung maßgeblich bestimmen kann“, sei freilich „sowohl für das gerichtliche als auch das schiedsgerichtliche Verfahren“ zu vermeiden. ${ }^{517}$ Im ICSID-Regelwerk wird das Thema jetzt explizit, wenn auch nur punktuell aufgegriffen: Zum einen werden bei Verfahrensbeginn nähere Angaben zu einer Drittfinanzierung verlangt (Arbitration Rule 14), zum andern kann ,third party funding“ ein Grund (allerdings nicht der alleinige) dafür sein, dass vom Schiedsgericht eine ,security for costs“ gefordert wird (Arbitration Rule 53 Abs. 4 S. 2). ${ }^{518}$

\section{G. Wieviel "Afrikanisierung" ist nötig und sinnvoll?}

\section{Differenzierende Internationalisierung oder (Re-)Nationalisierung?}

Insgesamt scheint einerseits eine allmähliche Öffnung der ICSID- und weiterer global angelegter Streitbeilegungsmechanismen für andere als traditionelle Schiedsorte (außerhalb des europäisch-nordatlantischen Raums) und für Mediatoren, Schlichter oder Schiedsrichter aus Asien, Lateinamerika und nicht zuletzt aus Afrika vor sich zu gehen, zugleich aber auch zunehmend neue Einrichtungen im Süden geschaffen zu werden, die dann sowohl Kontinent übergreifend kooperieren als auch ihre Dienste letztlich weltweit anbieten. Insoweit treffen dabei Globalisierungs- und Regionalisierungstendenzen zusammen und schließen sich nicht aus, sondern eröffnen gerade auch Investoren eine größere Wahl einer ,passenden“ Einrichtung. Dabei besteht eine Vielzahl von intergouvernementalen und national verankerten Institutionen und Verfahren, die inhaltlich und prozedural jedoch ungeachtet ihrer eher privat- oder öffentlich-rechtlichen Regularien oft sehr ähnlich gestaltet sind, so dass insoweit eine Konkurrenz mit Hinblick auf „gute“ Qualität nicht ohne weiteres stattfinden kann, sondern auch von adäquater Ressourcenausstattung abhängt. In der Sache werden sich im Hinblick auf für die jeweiligen Streitparteien wesentliche Fragen daher ,best practices“" entwickeln, insbesondere ${ }^{519} \mathrm{zu}$,,arbitrator ethics, conduct and qualifications“, ,reducing the risk of frivolous and unfounded cases“, ,allowing claims to proceed (filter)“" sowie „appellate mechanism and consistency of rulings“. Überaus bedeutsam sind daneben The-

517 BT-Drs. 18/1120, 11; dazu auch Gaukrodger/Gordon (Fn. 473), 36 ff.; McKenny, in: Legum (Fn. 88), $137 \mathrm{ff}$.

518 Ebenso (Additional Facility) Arbitration Rule 63 (Abs. 4 S. 2).

519 Angelehnt an das 2013/14 zu öffentlicher Konsultation gestellte Dokument der EU-Kommission (tradoc152280) über ,,modalities for investment protection and ISDS in TTIP“; ähnlich Gaukrodger/Gordon (Fn. 473), 17 ff.; Bundesverband der Deutschen Industrie, Investitionsschutzabkommen und Investor-Staat-Schiedsverfahren, 2015, $48 \mathrm{ff}$. 
men wie der Umgang mit „,multiple claims“ und die „relationship to domestic courts“, allgemein wichtig die Austarierung von angemessener ,transparency in ISDS“. Und schließlich ist es aus konstitutioneller Sicht entscheidend zu klären, ob und wie weit Streitbeilegung extern determiniert werden darf und soll, vor allem durch, guidance by the Parties on the interpretation of the agreement". So mögen eher tatsächliche Hürden für eine stärkere „Afrikanisierung“ bei ISDS ${ }^{520}$ existieren als solche rechtlicher Natur, zumal berechtigte Wünsche nach ,diversity“ weithin schon im Rahmen von Bestellungs- und Befangenheitsregeln verwirklicht werden könnten und unabhängig-unparteiische Entscheidungsfindung durch mehr Politisierung gefährdet würde. ${ }^{521}$

Insofern muss es, trotz der unbestrittenen Vorteile gegenüber dem Status Quo, nicht höchste Priorität sein, einen ständigen (regionalen oder gar globalen) Multilateral Investment Court zu errichten, wie dies vor allem von der EU-Kommission nachdrücklich forciert wird ${ }^{522}$ und schon in einigen von ihr geschlossenen (freilich jeweils bilateralen) völkerrechtlichen Abkommen Erwähnung gefunden hat. ${ }^{523}$

Ähnlich zu bewerten sind auch bislang nur in der Fachdiskussion formulierte Vorstellungen von der Gründung einer World Investment Organization ${ }^{524}$ oder eines International Investment Advisory Center. ${ }^{525}$ Ernst zu nehmen und aufzugreifen sind „concerns“, wie sie Mali im Herbst 2019 einer UNCITRAL-Arbeitsgruppe vortrug, in Bezug auf „lack of con-

520 Vgl. etwa Le Bars, 11.5.2018, https://globalarbitrationreview.com/review/the-middle-eastern-and -african-arbitration-review/the-middle-eastern-and-african-arbitration-review-2018/article/the-ev olution-of-investment-arbitration-in-africa; Chidede, Oregon Review of International Law 20 (2019), 437 (467 f.); Gramlich, RiA 2020, 3 (30).

521 Vgl. Kinnear/Le Cannu, ICSID Review - Foreign Investment Law Journal 34 (2019), 542 ff.; ferner Gaukrodger Gordon (Fn. 473), 17 ff.; Honlet et al. (Fn. 369), 14 f.; Mbengue und El Kady/ de Gama, ICSID Review - Foreign Investment Law Journal 34 (2019), 455 ff., 482 ff.; Knieper, SchiedsVZ 2020, $60 \mathrm{ff}$.

522 Vgl. das „concept paper“ der Kommission (tradoc 153408), Investment in TTIP and beyond the path for reform; Rat der EU, Negotiating directives, Rats-Dok. 12981/17 ADD 1 DCL 1, 20.3.2018; dazu etwa Alvarado Garzon, ZEuS 2019, 477 (478 ff.); Feichtner und Krajewski, in: Ludwigs/Remien (Fn. 57), 97 ff., 113 ff.; ähnlich der von Krajewski 2015 für das deutsche Bundeswirtschaftsministerium entworfene Modellvertrag in Art. 16 ff.; kritisch dazu Sandrock, RIW 2015, 625 ff.; ablehnend BT-Drs. 19/97 v. 22.11.2017, 1 ff. (Antrag DIE LINKE); BT-Drs. 18/9621 v. 13.9.2016, 1 ff. (Antrag BÜNDNIS 90/DIE GRÜNEN); ferner Holterhus, Stellungnahme zur Bundestags-Anhörung, 6.6.2018, 3 ff.; Bungenberg/Reinisch, From bilateral arbitral tribunals and investment courts to a multilateral investment court, European Yearbook of International Economic Law, Special issue, 2020; Reinhold, EuZW 2020, $589 \mathrm{ff}$.

523 BT-Drs. 18/13645 v. 27.9.2017, 11 (Bundesregierung zu Verhandlungen eines EU-Investitionsschutzabkommens mit Myanmar); ablehnend daher zum EU Investment Protection Agreement mit Vietnam (http://trade.ec.europa.eu/doclib/press/index.cfm?id=1437) BT-Drs. 19/7060 v. 16.1.2019, 2 f. (Antrag BÜNDNIS 90/DIE GRÜNEN); zum Gegenvorschlag dieser Fraktion BTDrs. $19 / 5908$ v. $20.11 .2018,1$ ff.

524 Vgl. Butler/Subedi, Neth. Int. Law Rev. 64 (2017), 43 ff.

525 Vgl. Sharpe, 26.7.2019, https://www.ejiltalk.org/an-international-investment-advisory-center-bey ond-the-wto-model. 
sistency, coherence, predictability and correctness" von ISDS-Entscheidungen, ,imbalance in investment treaties“, ,lack of expertise and preparation“ sowie $\mathrm{zu}$,arbitrators and decision makers" und zu „cost and duration of ISDS cases“.526

Ein schrittweises Vorgehen bei einer IIA- wie einer ISDS-Reform ${ }^{527}$ ist demgegenüber der geeignetere und wohl auch alternativlose Weg, solange eine Lösung oder zumindest Verbesserung der „Architektur“ ${ }^{\text {“528 }}$ auf internationaler Ebene weiterhin von einer großen Mehrheit der Staatengemeinschaft angestrebt wird. Auch das im November 2020 unterzeichnete Abkommen über eine Regional Comprehensive Economic Partnership, dem neben den 10 ASEAN-Mitgliedern auch Australien und Neuseeland sowie Japan, Südkorea und die Volksrepublik China angehören, enthält ein Kapitel zu ,investment" mit einem „, built-in work programme on investor-state settlement disputes provisions“ ${ }^{\text {" } 529}$ die elf Teilnehmer umfassende Comprehensive and Progressive Trans-Pacific Partnership (2018) im inhaltlich entsprechenden Kapitel überdies einen detaillierten Unter-Abschnitt mit ,,modernen“ ISDS-Regeln (Art. 9-18 - Art. 9.30). ${ }^{530}$

Denn seit einigen Jahren zeigen sich nicht nur in unterschiedlichsten Staaten, sondern auch bei Wissenschaftlerinnen und Wissenschaftlern Tendenzen einer (Re-)Nationalisierung. Bereits 2014 formulierte van Harten: „The clearest and most defensible step is to remove investor-state arbitration from the treaties. The exceptional use of arbitration to resolve disputes about sovereign conduct and access to public funds is flawed due to the lack of the usual safeguards of independence, openness, and fairness and the lack of balance in the allocation of rights and responsibilities ${ }^{6.531}$ solche Einschätzung ist nicht nur vereinzelt anzutreffen.

526 U.N. General Assembly, Dok. A/CN.9/WG.III/WP.181 v. 17.9.2019, 2 f. Berücksichtigung sollten auch die in Schiedsverfahren üblichen Sprachen und ihr Verhältnis zu den Sprachen der Streitparteien finden.

527 Vgl. UNCTAD, Issues Note 2, Juni 2017 (Phase 2 of IIA reform: Modernizing the existing stock of old-generation treaties): UNCTAD, IIA Issues Note 1, März 2019 (Reforming investment dispute settlement: a stocktaking); UNCTAD, IIA Issues Note 1, Juli 2020 (The changing IIA landscape); UNCTAD, World Investment Report 2020, 106 ff., 210 ff.; UNCTAD, SDG Investment Trends Monitor, 2019; ferner Knieper, SchiedsVZ 2015, 25 ff.; Muchhala, U.N. General Assembly, A/HRC/WG.2/19/CRP.5, 19.4.2018; Gramlich/Conen, SZIER 26 (2016), 391 (402 ff.).

528 Vgl. Vinuales, International Investment Law and Natural Resource Governance, Sept. 2015, 1 (ff.), www.e15initiative.org/.

529 https://asean.org/storage/2020/11/Summary-of-the-RCEP-Agreement.pdf; dazu UNCTAD, Investment Trends Monitor, Special Issue, Nov. 2020.

530 https://www.mfat.govt.nz/assets/Trade-agreements/TPP/Text-ENGLISH/9.-Investment-Chapter.p df.

531 „Comment“ im Rahmen der öffentlichen Konsultation zu Investor-Staat-Schiedsverfahren in TTIP, 3.7.2014, 54 f.; ähnlich Sinclair, Investor-State Dispute Settlement in the TTIP and CETA, Juli 2014, 3, 12; Bernasconi-Osterwalder, Reply to the European Commission's Public Consultation ..., Juni 2014, 29; ferner Krajewski, Zu Investitionsschutz und Investor-Staat-Streitbeilegung im Transatlantischen Handels- und Investitionspartnerschaftsabkommen (TTIP), Mai 2014, 14 f.; BT-Drs. $18 / 3747$ v. 14.1.2015, 2 f. (Antrag BÜNDNIS 90/DIE GRÜNEN); ähnlich Däubler- 


\section{Stärkung demokratisch-rechtsstaatlicher Legitimität}

Soweit sich Verfahrensregeln und auch Vorgaben für die Bestellung (und Abberufung) von Schiedsrichtern wie anderen Intermediären fachlich-inhaltlich weithin bewährt haben und prinzipiell als angemessen empfunden werden, ist gleichwohl Kritik von unterschiedlichen Seiten und Disziplinen Rechnung zu tragen, die auf zu wenig transparentes Zustandekommen von Regelungen (einschließlich der Änderungen), die unzulängliche und verspätete Einbindung der (nationalen, aber auch „supranationalen“"532) Parlamente und in der Praxis auftretende Konstellationen von ,bias“ abzielen, durch die generell wichtige „Regulierungs"-Belange nicht hinreichend beachtet werden; wo justiziable Maßstäbe fehlen oder defizitär sind, könnten solche prozeduralen Verbesserungen (zusätzliche) Legitimität schaffen. ${ }^{533}$

Inhaltlich wäre daher eine breitere und tiefere Konstitutionalisierung (vor allem der Regelerzeugung ${ }^{534}$ ), insbesondere bei intergouvernementaler Rechtsetzung durch inter- und spezielle supranationale Organisationen nötig. Einher gehen müsste dieser Prozess mit Maßnahmen zur (funktionellen) Konsolidierung ${ }^{535}$ im Hinblick auf Regelungsebenen wie auf Streitbeilegungsinstitutionen und -mechanismen, ohne dass freilich eine förmliche Konzentration auf einige wenige Stellen anzustreben wäre. Für die generelle Akzeptanz von (schieds)richterlichen wie (anderen) ADR-Entscheidungen ist wegen der inhärenten Schwächen zwangsweiser Durchsetzung „diversity“"536 von Personen, Orten und Einrichtungen existenznotwendig und bedarf es der Einbeziehung auch afrikanischer Regelungen und Praxiserfahrungen (rechtskulturell, rechtspolitisch und institutionell). Jedoch bedeutet dies nicht etwa eine „, Afrikanisierung“ oder, bezogen auf andere Weltregionen, eine „Kontinentalisierung“ der (Investor-Staat-)Streitbeilegung; ähnlich wie nationale Ballungsräume oder

Gmelin, in: Voß (Fn. 57) 32 (33 f.); zuspitzend Eberhardt, The zombie ISDS, Febr. 2016, 35, $36 \mathrm{ff}$.

532 Soweit parlamentarische Versammlungen dort überhaupt bestehen (oben, Fn. 422) und zudem auch organisationsrechtlich diesbezüglich Informations- und Mitentscheidungsrechte haben; vgl. dazu Art. 218 Abs. 6, 10 AEUV; dazu Schlussanträge GA'in Kokott, 28.102015, Rs. C- 263/14, Rn. 76 ff., EuGH, 14.6.2016, Gutachten, Rs. C-263/14, Rn. 68 ff., ECLI:EU:C:2016:435.

$533 \mathrm{Zu}$ einseitig daher der Ansatz im Background Document zum OECD Workshop, 16.10.2019, Certifying reform efforts through international standards for Compact with Africa countries, $5 \mathrm{ff}$., https://www.compactwithafrica.org/content/dam/Compact\%20with\%20Africa/events/OECDWor kshop/Background $\% 20$ document $\% 20-\% 20$ International $\% 20$ Standards $\% 20$ for $\% 20$ Compact $\% 20 \mathrm{a}$ nd\%20G20\%20 countries.pdf.

534 Zumindest ansatzweise (,inclusive“) auch World Development Report 2019, 115.

$535 \mathrm{Zu}$,treaty shopping“ etwa Klodt/Lang, Wirtschaftsdienste 2015, $482 \mathrm{ff}$.

536 Vgl. etwa Engle, https://africaarbitration.org/2018/06/11/colonialism-and-international-arbitratio n-looking-toward-africa-by-philip-w-whit-engle-fciarb/; Adekoya, https://africaarbitration.org/20 17/11/07/is-international-arbitration-truly-iinternational-the-role-of-diversity/. 
„special economic zones“537 muss ein Schwerpunkt der Neu- und Umgestaltung hier auf spezifischen (grenzüberschreitenden) Wirtschaftsräumen liegen, seien diese subregional oder aber umgekehrt mit anderen Weltregionen verknüpft oder zumindest aufeinander abgestimmt. ${ }^{538}$ Regelsetzung und -durchsetzung erfordert hier einen für institutionelle, territoriale und sachlich-inhaltliche Erweiterung offenen intergouvernementalen normativen Rahmen, eine letztlich global (und nicht nur von G7 oder G 20) konzipierte „Architektur“, die allerdings auch eine Einbindung bestehender wie neuer "privater" Streitbeilegungszentren ermöglicht. Solange und soweit dafür noch keine rechtsverbindlichen multilateralen Übereinkommen bestehen, wären ,weichere“ Formen der Kooperation zwischen einzelnen Akteuren auf internationaler Ebene wichtig, ${ }^{539}$ die aber speziell auf der Stufe eines ,enforcement" durchaus rechtserheblich sein müssten, damit die erreichten ,settlements" nicht fruchtlos bleiben.

537 Vgl. UNCTAD, World Investment Report 2019, 127 ff.; Badawy, 8.9.2910, https://afaa.ngo/page18097/7869606; krit. Henn, Die Illusion der Souveränität. Sonderwirtschaftszonen und Sondersteuern in Afrika, 2020, $8 \mathrm{ff}$. und (zu Senegal) $25 \mathrm{f}$.

538 Diverse Optionen in UNCTAD, UNCTAD`s reform package for the international investment regime, 2018, 29 ff., 97 ff.; zum „myth of harmony“ Gaillard, ICSID Review - Foreign Investment Law Journal 34 (2019), $553 \mathrm{ff}$.

539 Zum OECD-Konzept der Kontaktstellen oben, D.V. 